\title{
"Toolbox" for the Processing of Functional Polymer Composites
}

Cite as

Nano-Micro Lett.

(2022) $14: 35$

Received: 28 September 2021

Yun Wei ${ }^{1}$, Hongju Zhou ${ }^{1}$, Hua Deng ${ }^{1}{ }^{凶}$, Wenjing $\mathrm{Ji}^{1}, \mathrm{Ke} \mathrm{Tian}^{1}$, Zhuyu Ma $^{1}$,

Accepted: 17 November 2021

Kaiyi Zhang ${ }^{1}$, Qiang Fu ${ }^{1}$

Published online: 16 December 2021

(C) The Author(s) 2021

\section{HIGHLIGHTS}

- The processing methods of functional polymer composites (FPCs) are systematically summarized in "Toolbox".

- The relationship of processing method-structure-property is discussed and the selection and combination of tools in processing among different FPCs are analyzed.

- A promising prospect is provided regarding the design principle for high performance FPCs for further investigation.

\begin{abstract}
Functional polymer composites (FPCs) have attracted increasing attention in recent decades due to their great potential in delivering a wide range of functionalities. These functionalities are largely determined by functional fillers and their network morphology in polymer matrix. In recent years, a large number of studies on morphology control and interfacial modification have been reported, where numerous preparation methods and exciting performance of FPCs have been reported. Despite the fact that these FPCs have many similarities because they are all consisting of functional inorganic fillers and polymer matrices, review on the overall progress of FPCs is still missing, and especially the overall processing strategy for these composites is urgently needed. Herein, a "Toolbox" for the processing of FPCs is proposed to summarize and analyze the overall processing strategies and corresponding morphology evolution for FPCs. From this perspective, the morphological control methods already utilized for various FPCs are systematically reviewed, so that guidelines or even predictions on the processing strategies of various FPCs as well as multi-functional polymer composites could be given. This review should be able to provide interesting insights for the field of FPCs and boost future intelligent design of various FPCs.
\end{abstract}

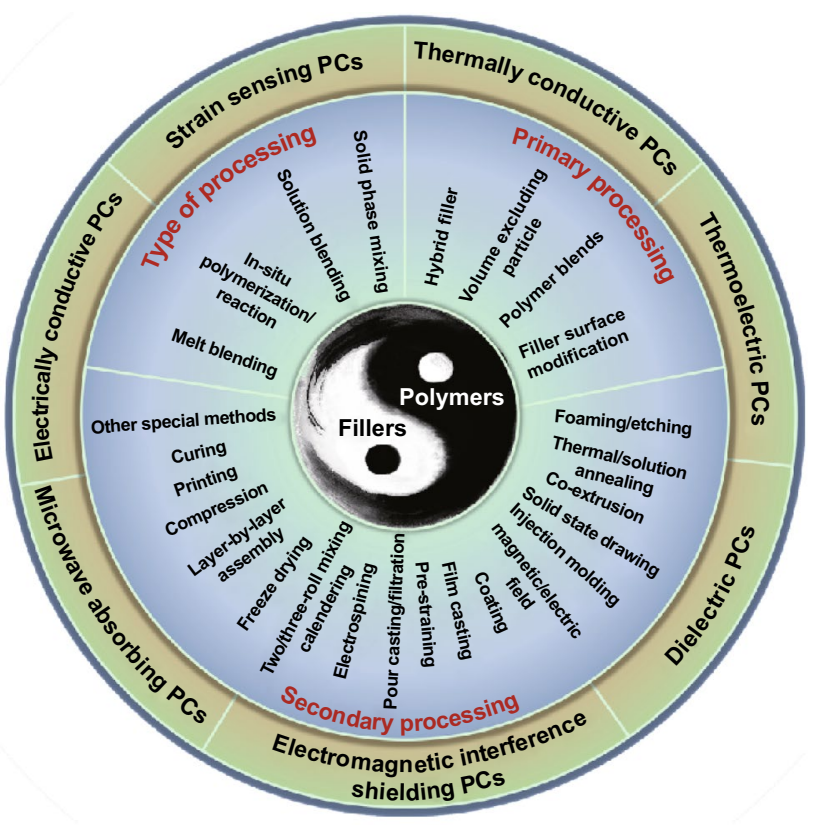

KEYWORDS Toolbox; Functional polymer composites; Processing strategy; Morphology control

Yun Wei and Hongju Zhou have contributed equally to this review article.

$\triangle$ Hua Deng, huadeng@scu.edu.cn

${ }^{1}$ College of Polymer Science and Engineering, State Key Laboratory of Polymer Materials Engineering, Sichuan University, Chengdu 610065,

People's Republic of China 


\section{Introduction}

Functional polymer composites (FPCs) have gained enormous attention in recent decades owing to their great potential in delivering a wide range of functionalities, e.g., electrical conductivity [1-3], strain sensing [4, 5], thermal conductivity [6-8], thermoelectric property $[9,10]$, dielectric property $[11,12]$, electromagnetic shielding (EMI) [13-15] and microwave absorption [16]. These functionalities are largely determined by physicochemical properties of functional fillers (i.e., size and shape of fillers) and their network morphology in polymer matrices. Therefore, as shown in Fig. 1, numerous research efforts have been devoted to the optimization of these functionalities through morphology control and interfacial modification, where a wide range of preparation methods and exciting performance of FPCs have been reported [17-21]. Despite the fact that these FPCs have many similarities because they all consist of functional inorganic fillers and polymer matrices, reviews on the progress of FPCs are limited in individual FPCs field, while overview on the overall progress of FPCs is stilling lacking, and especially the overall processing strategies for these composites is urgently needed.

As well known, the structure-property relationship is a key guidance in designing polymeric composites. Furthermore, tailoring structure of FPCs by morphology control and interfacial modification finally footholds on the selection and combination of preparation methods in practice. In this regard, appropriate selection and combination of preparation methods is a prerequisite to enable the optimization of functionalities. To date, most of researchers merely focus on the morphology control of filler network and modification of the interface between filler and polymer [22, 23], while the relationships between different processing methods, structure of FPCs (including filler network morphology and interface between filler and polymer) and final functionalities are still not clear. Meanwhile, researchers tend to focus on their own specific field [24]. Nevertheless, finding new inspiration from other mainstream or specific processing methods in different FPCs fields may pave a novel potential way for the preparation of high performance FPCs.

However, there are many processing methods for preparing FPCs, not to mention the large number of combinations between different methods. It is actually not easy to select the most suitable processing methods for wide range of FPCs.
Therefore, efficient classification and summary strategy is vital to systematically study processing methods and processing method-structure-property relationship. After considering above issues, the concept of "Toolbox" is proposed based on regarding one or several similar processing methods as a kind of tool for designing various FPCs. Previous work from our group with some specific tools for the morphology control "Toolbox" has been reported [25]. To extend the tools for various FPCs and summarize the "Toolbox" more comprehensively, the processing and fabrication of various FPCs are summarized for wider range of literature. Then, a systematic "Toolbox" is developed, which covers recent progress on processing methods for better designed functionalities as shown in Table 1. To better understand these processing methods, they are firstly categorized into various processing types: melt blending, in situ polymerization and solution blending. Furthermore, specific processing methods are divided into primary processing tools and secondary processing tools according to the sequence they are utilized, which will be listed in detail in Sect. 2. Thereinto, primary processing tools include hybrid filler, volume excluding particle, polymer blends and filler surface modification, while secondary processing tools have more species, such as foaming, thermal annealing, injection molding, magnetic field, coating, film casting, pre-straining and electrospinning. Therefore, such "Toolbox" could be used to not only study the relationship of processing method-structure-property, but also possibly create novel approaches for the preparation of certain FPCs by combining a number of exiting tools, or adapting tools from other type of FPCs. Meanwhile, newly emerging designed processing tools could also be incorporated into such "Toolbox" to update and enrich the system.

This review provides a comprehensive overview on the todate processing methods for preparing FPCs, including electrically conductive polymer composites (ECPCs), strain/pressure sensing polymer composites (SSPCs), thermally conductive polymer composites (TCPCs), dielectric polymer composites (DEPCs), electromagnetic interference (EMI) shielding polymer composites (EMISPCs), microwave absorbing polymer composites (MAPCs) and thermoelectric polymer composites (TEPCs). The basic mechanism and background for above FPCs can be found in Supporting Information and a number of decent review papers [23, 26-34], respectively. In this review, we start with the details and characteristics of different processing methods and then summarize diverse selections and 
(A)

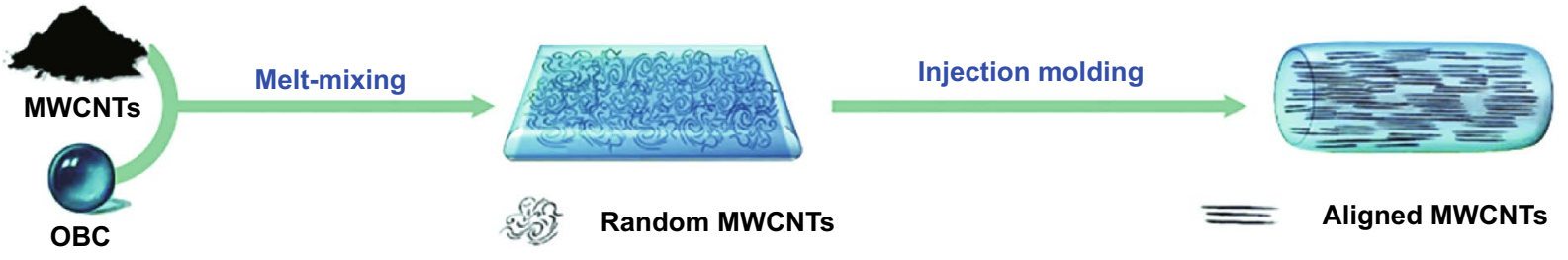

(B)
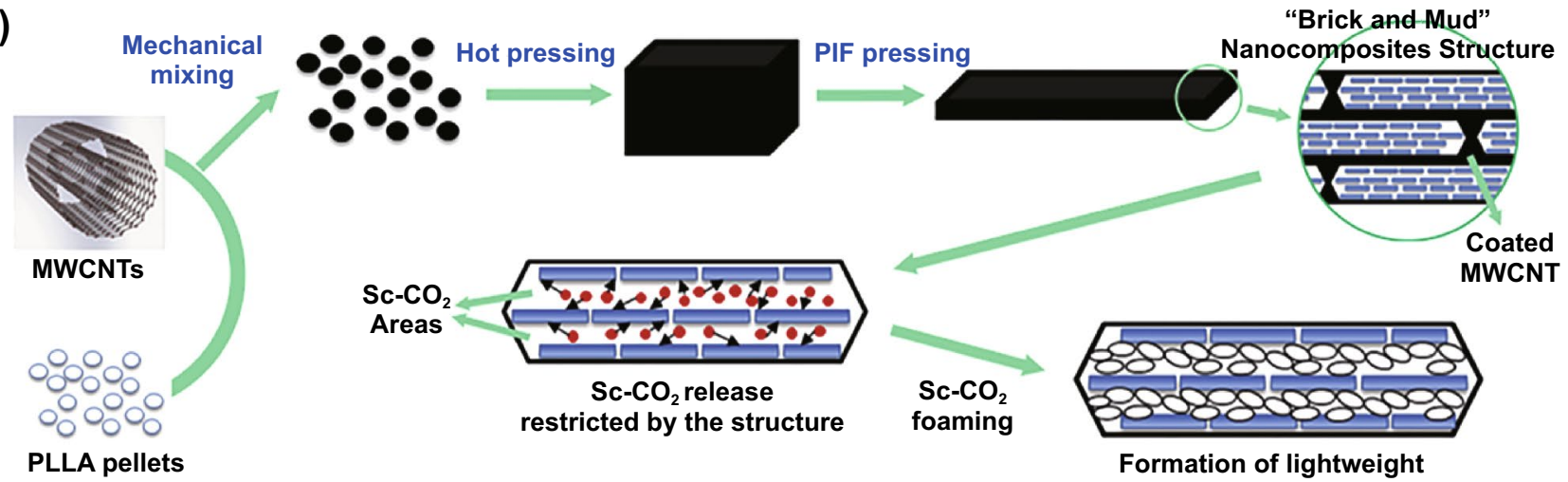

PLLA pellets

(C)
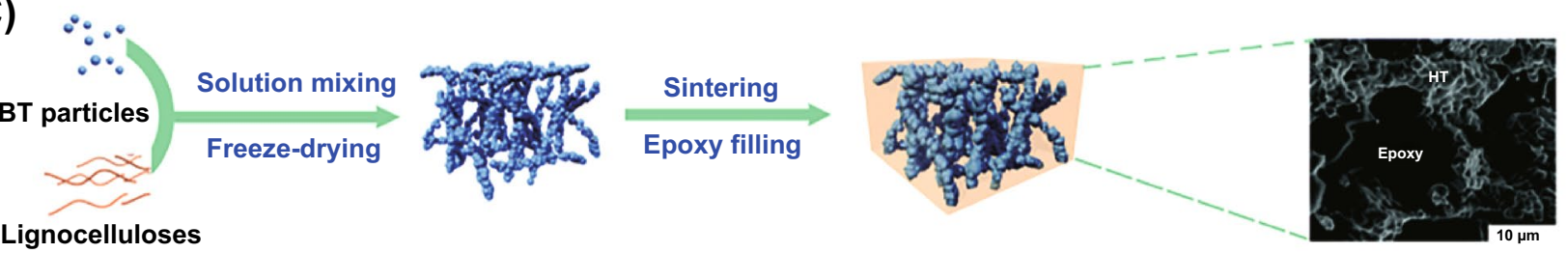

(D)

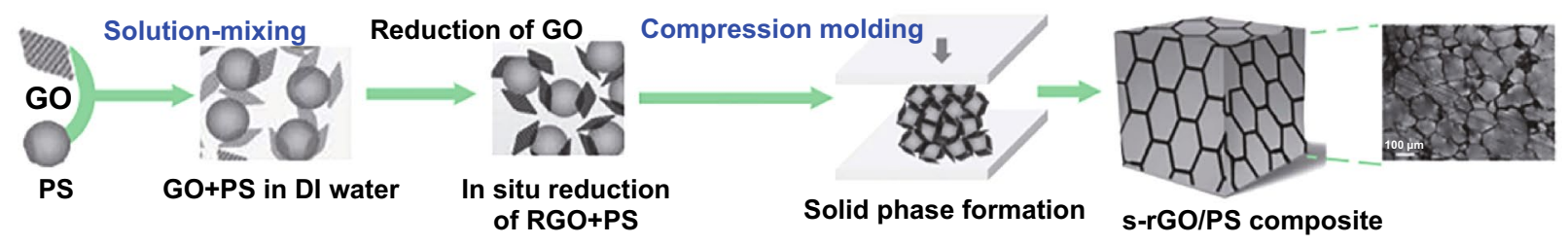

(E)

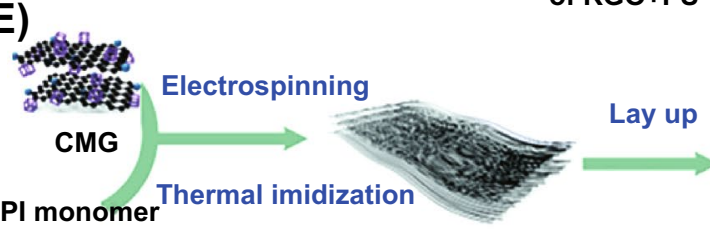

CMG/PI fibers

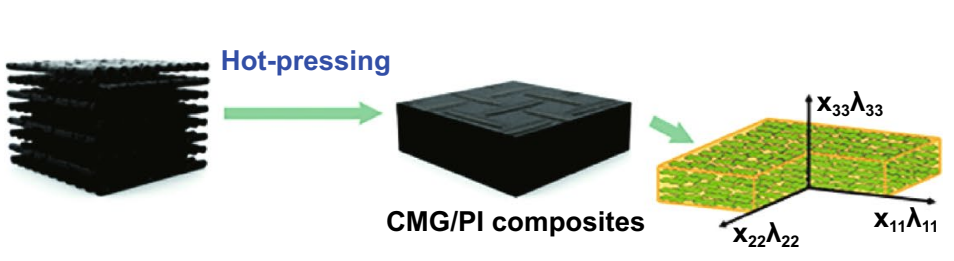

Fig. 1 A Schematic for the preparation and morphology of ethylene- $\alpha$-octene block copolymer (OBC)/multi-walled carbon nanotubes (MWCNTs) via melt blending and injection molding. Reprinted with permission from Ref. [17]. B Schematics of using pressure-induced flow (PIF) and supercritical carbon dioxide $\left(\mathrm{Sc}-\mathrm{CO}_{2}\right)$ to prepare poly(l-lactide) (PLLA)/MWCNTs foams. Reprinted with permission from Ref. [18]. C Schematic illustration of the epoxy/BaTiO 3 (BT) composites fabrication process through solution blending BT with lignocellulose, freeze drying, sintering and filling with epoxy in sequence. Reprinted with permission from Ref. [19]. D Schematic of the fabrication and structure reduced graphene oxide/polystyrene (rGO/PS) composites by in-situ reduction following high-pressure solid phase formation. Reprinted with permission from Ref. [20]. E Schematic diagram for the fabrication of chemically modified graphene/polyimide (CMG/PI) nanocomposites with laminate structures by electrospinning-hot pressing method. Reprinted with permission from Ref. [21] 
combinations of processing methods. Furthermore, the structural features brought to the filler networks by these processing methods are also discussed. Additionally, the future application trend of such "Toolbox" is prospected. By combining with "artificial intelligence" computer program, such concept could be used to collect and summarize the overall materials database, and thus, possible processing routes for given structure or targeted properties could be predicted. Such method could be used to guide the future preparation of FPCs to achieve outstanding performance more efficiently and effectively.

\section{Toolbox}

Utilizing inorganic filler and polymer could bring composites with exciting functional properties. However, converting the potential properties of fillers and polymers into composites is not easy. Potential filler agglomeration, filler network distribution in the polymer matrix, the interface between filler and polymer, and the morphology and structure of the entire composite would largely influence the final properties, which pose more stringent challenge to the design strategy and processing method. In the past decade, a large number of reports were focused on morphology control to improve the performance of various composites. This process is realized by different processing methods either individually or collectively. On the one hand, different processing methods have unique processing conditions or implementation methods, and their principles, methods, influencing issues are different to result in different outcome. On the other hand, different processing methods may also lead to similar morphology. For example, porous materials could be achieved by foaming, freeze-drying or even 3D printing. Herein, a concept of "Toolbox" for the processing of FPCs is proposed to summarize and analyze the overall processing strategies for a range of FPCs. As shown in Table 1, the "Toolbox" consists of three parts: the type of processing, primary processing and secondary processing. These types of processing are the major type of processing methods summarized for different literature. Then, primary processing methods can be considered as the materials selection and mixing

Table 1 "Toolbox": the type of processing and specific tools used during processing for morphology control. These tools are summarized from 700 literature collected in various FPCs fields

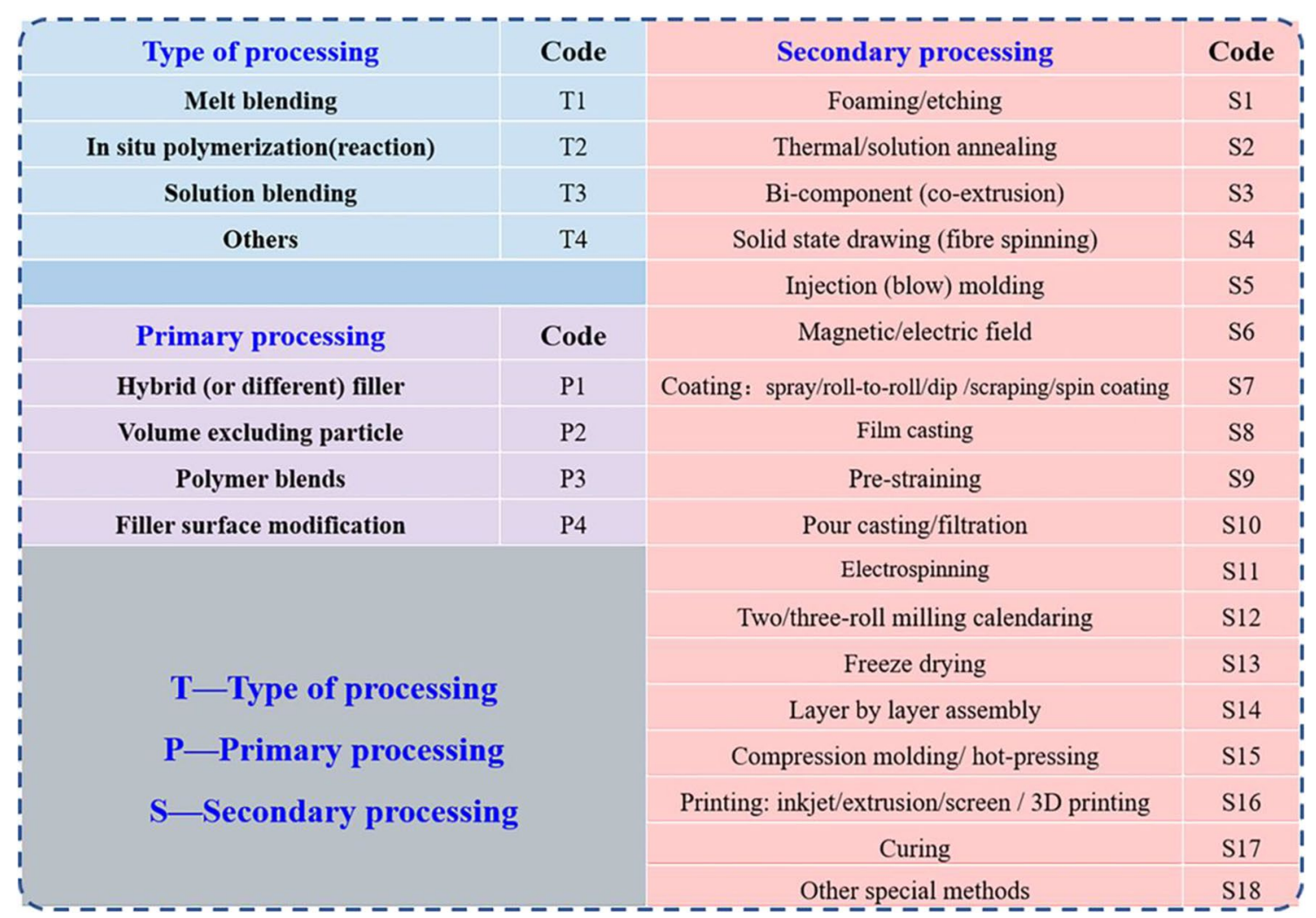


procedure before processing. Finally, secondary processing methods process various materials into desired shape and structure. All these methods have important influence on the final structure and properties of FPCs. The methods and characteristics of these processing strategies are discussed in the following part.

\subsection{Type of Processing}

\subsubsection{Melt Blending}

Melting blending is based on the fact that thermoplastic polymers become soft upon being heated up, while the properties of polymers are largely remained the same after they are cooled down. This technique requires relatively high processing temperature; typical amorphous polymers and semi-crystalline polymers can be processed above their glass transition temperature and melting temperature, respectively. Such process can be achieved by equipment, such as extrusion, internal mixing, injection molding and blow molding, which are capable of being operated at elevated temperatures and generating high shear forces. Due to simplicity and availability of these processes, melt blending is considered as the most time-saving, cost-effective and scalable method for the production of FPCs for a wide range of polymer matrixes. This approach is free of solvents and contaminants, which are present in solution processing and in situ polymerization. Nevertheless, the main advantages of those two processing types are that the filler dispersion level could be achieved at molecular scale and provides an advantage of low viscosity, which can facilitate mixing and dispersion. Therefore, compared to solution mixing and in situ polymerization, the filler dispersion status of FPCs with melt blending is often less homogeneous. During mixing, a number of parameters such as temperature, rotation speed and mixing time must be fine-tuned to optimize the resulting properties [35]. During melt blending process, filler agglomerates generally undergo dispersion by erosion and rupture mechanisms simultaneously.

\subsubsection{In Situ Polymerization}

In the process of preparing FPCs by in situ polymerization, the functional fillers are uniformly dispersed in polymer monomers firstly, and then an initiator is added in the filler/ monomer system to initiate polymerization. The fillers often need to be surface modified with functional groups or polymer, so that there is a strong interface interaction between fillers and polymer matrix in composite prepared by in situ polymerization. The advantage of in situ polymerization is that inorganic fillers have rather homogeneous dispersion and potentially good interfacial strength between filler and polymer matrix, for which polymer molecules are either wrapped around or covalently bonded to these fillers. Moreover, in situ polymerization enables the grafting of polymer chains onto the surface of fillers, allowing the resulting FPCs with high filler loading level and excellent miscibility with polymer. In situ polymerization is usually used to process FPCs containing thermally unstable or insoluble polymers, which cannot be prepared by other strategies such as solution or melting compounding. Depending on monomer molecular structures, the required molecular weight and molecular weight distribution of the polymers, anionic, radical, ringopening and other polymerization reactions have been used. Moreover, with the continuous research and the development of in situ polymerization technology in FPCs, many methods, such as in situ intercalation polymerization of polymer in clay [36], template-directed in situ polymerization [37], in situ oxidation polymerization in reverse microemulsion [38] and interfacial adsorption-soft template polymerization [39], are developed to obtain unique structure and high-performance composites.

\subsubsection{Solution Blending}

Compounding techniques, like melt blending, can lead to industry-scale preparation, but it is difficult to realize locally homogeneous dispersion due to the filler-filler interaction, especially for fillers with high aspect ratio such as carbon nanotubes (CNTs), while in situ polymerization is required in relative high-viscosity systems. Thus, another technology, solution mixing, needs to be introduced for better filler dispersion. The solution blending method refers to dispersing inorganic filler in polymer solution using a suitable solvent and removing the solvent finally to obtain FPCs. In the solution blending system, uniform filler dispersion in polymer solution and lower viscosity are two typical aspects. Nanofillers usually cannot be directly dispersed well in most of the solvents due to their exceptionally high specific surface area resulted strong interactions [40]. In order 
to promote the dispersion of fillers, some additives were introduced in solution blending, such polydopamine and ionic liquid. Deng et al. reported a ionic liquid of 1-butyl3-(4-sulfobutyl) imidazolium trifluoromethanesulfonate was added into single-walled carbon nanotube/poly (3,4 ethylenedioxythiophene):poly-(styrenesulfonate) (SWCNT/ PEDOT:PSS) system [41]. On the one hand, the introduction of ionic liquid promotes the dispersion of SWCNT, and on the other hand, ionic liquid has ion-exchange effect which plays a similar role of high-boiling solvent effects to provide primary and secondary doping of PEDOT:PSS, to enhance the electrical conductivity via manipulation of the ordering structures of conducting polymers. Here is another way to adjust composite morphology and structure by blending filler and polymer matrix in solution blending. Liang et al. reported a morphology design strategy by mixing SWCNTs and polypyrrole (PPy) nanowires, and unique sandwiched layer structure free-standing PPy/SWCNT composite films were obtained [42].

Solution blending can be used to fabricate FPCs with excellent dispersion, and it is also a necessary step for most of the secondary process processing methods proposed such as coating, electrospinning [43] and printing technology [44], but the use of solvent retards their industrial scale production to some extent. There are a number of issues that could be adjusted during this processing, such as the type of solvent, type of shear applied during mixing, mixing sequence, choice of polymer as well as filler, rate of solvent evaporation. These issues could have important influence on the final structure and properties of various FPCs.

\subsubsection{Others}

Other types of processing mainly involve physical mixing method of polymers and fillers and the preparation of thermoset polymers, such as epoxy resin. Physical mixing was widely used to fabricate FPCs with selectively distributed functional nanofillers, i.e., segregated structure. In segregated FPCs, fillers are primarily located at the interfaces of polymeric granules instead of homogeneously distributed. The formation of segregated filler networks substantially relies on polymer matrices with exclusionary microstructure, where functional fillers are located in a constrained volume, thus enhancing the effective density of the filler network at limited loadings [45, 46]. For thermoset polymers, such as epoxy resins, unique epoxide functional groups can react with a vast of hardener or curing agents at an elevated temperature to construct multiple cross-linking networks, resulting in good adhesive, mechanical, chemical and temperature resistance properties $[47,48]$.

\subsection{Primary Processing Method}

\subsubsection{Hybrid (or Different) Filler}

The hybrid filler method refers to adding two or more types of fillers into polymer. On the one hand, the simple superposition of multiple fillers could allow the composites to have the performance of all fillers at the same time, thereby achieving multiple functions. For example, mixing nano-barium titanate and nickel hydroxide can prevent the agglomeration of nano-barium titanate, thereby adjusting the contradictory relationship between dielectric constant and loss in dielectric materials [49]. On the other hand, with the combination of different dimensional topography fillers, a special structure is achieved, thus obtaining a synergistic effect. For instance, single-walled CNTs (MWCNTs) can effectively serve as thermal conduction bridge between graphene oxide (GO) and three-dimensional particles, reducing thermal resistance. As shown in Fig. 2A, MWCNTs which around the GO nanoplatelets can lead to the formation of a heat transport path. Moreover, the MWCNTs on the GO surfaces illustrate synergistic effect on the thermal transport properties due to their 3D network structures [50]. It can be seen that the main characteristic of hybrid (or different) filler is to use fillers with different dimensions, such as $0 D$ and 1D linear, one-dimensional linear and 2D sheets, to build a more complex 3D spatial structure.

\subsubsection{Volume Excluding Particle}

As the name implies, volume excluding effect means that due to the addition of fillers, one of the fillers occupies relative large volume and thus illustrates repelling effect on the other components in the system. This results in the other filler exhibiting some special distribution, thus achieving special functionality. This repulsive effect usually requires a large size difference or shape difference for the filler. At present, this method is mainly used to prepare segregated 
structure or selectively distributed network, so as to achieve low filler content and high performance. For example, using this method to construct a segregated structure can effectively decrease the filler content needed for achieving conductive networks [51, 52]. For instance, after expanded graphite and ultra-high molecular weight polyethylene (LLDPE) are mixed together by grinding and crushing, compression molding process is used to construct segregated structure (Fig. 2B) [53]. The percolation threshold of such structure is reduced, and other comprehensive properties of the composites can also be optimized [54].

\subsubsection{Polymer Blends}

Polymer blends method refers to two or more polymers that are mixed together to achieve functionality and performance improvement through constructing certain phase morphology. Common polymer blends structures include island-inthe-sea structures, bi-continuous structures, interpenetrating network structures and multilayer structures [55]. It is well known that the phase morphology of a mixture is mainly influenced by two factors, namely thermodynamic factors and kinetic factors. Thermodynamic factors include compatibility and interfacial tension between two phases. It is often observed that better diffusion and uniform mixing among different phases is achieved with better compatibility and greater binding force. Methods to promote compatibility mainly include chemical modification, addition of compatibilizers and formation of interpenetrating network or hydrogen bonding. By controlling process parameters (flow parameters, solvents, temperature, etc.), the phase transition process can be achieved. This special phase morphology brings large performance difference in polymer materials. For example, after adding CNTs to thermoplastic polyurethane (TPU) and olefin block copolymer (OBC) blends with bi-continuous structure (Fig. 2C), composites with enhanced strain sensitivity can be effectively obtained [56]. In addition, it is more noteworthy that structurally special blends, such as multilayer extrusion, multilayer injection molding and gradiently distributed filler, could also be obtained using some special processing methods and instruments. The disadvantage is that the controllable range of properties are often between two blended polymers, and the controllability decreases when multiple materials are used. It should also be
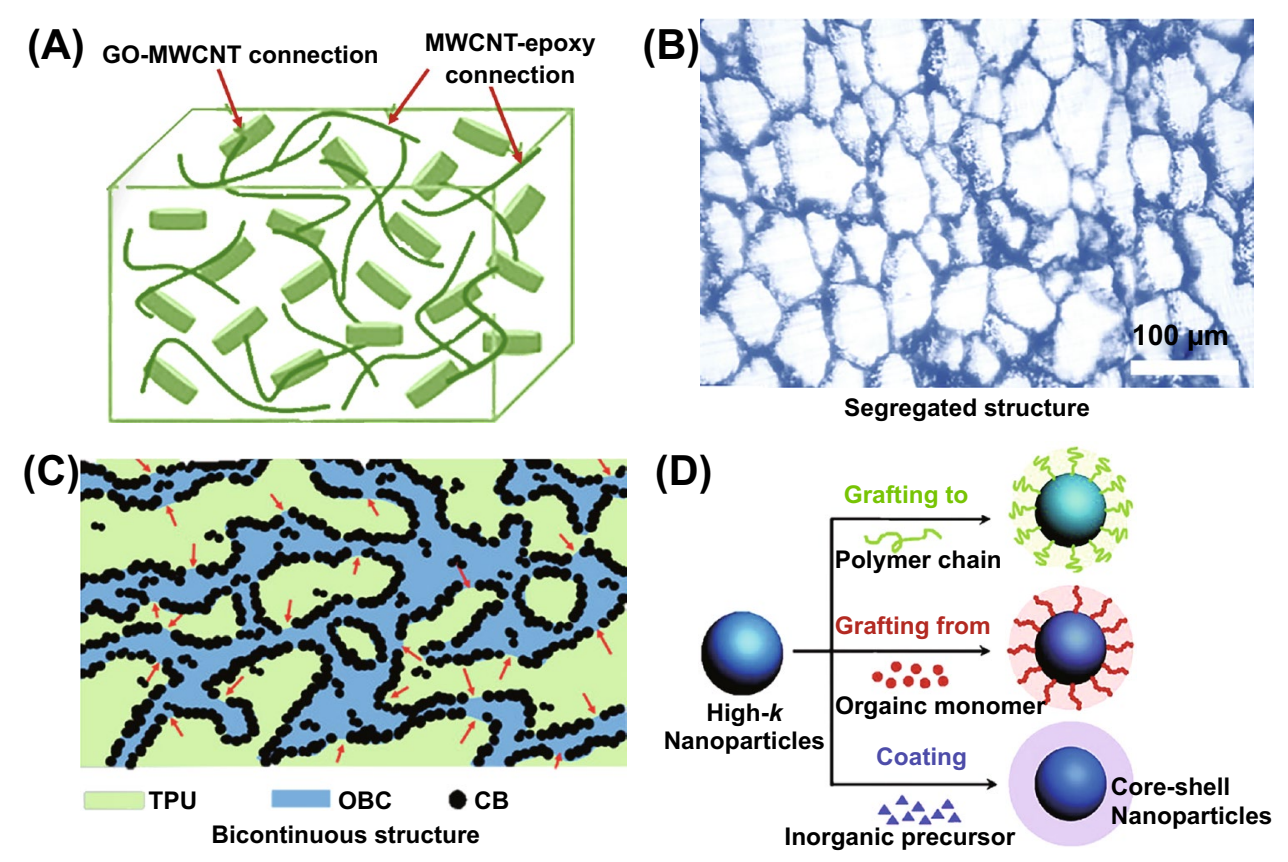

Fig. 2 A The construction of network structure with hybrid fillers containing graphene oxide/multi-walled carbon nanotube (GO/MWCNT)/ epoxy composite. Reprinted with permission from Ref. [50]. B Filler particles are localized at the boundaries between the polymer grains to form segregated system. Reprinted with permission from Ref. [53]. C Graphical representation of the network morphology evolution for olefin block copolymer/carbon black/thermoplastic polyurethane (OBC/CB/TPU)- ternary blends composites. Reprinted with permission from Ref. [56]. D General methods associated with filler surface modification. Reprinted with permission from Ref. [62] 
mentioned that the use of more than two types of polymers can sometimes generate interesting phase morphology, such as the core-shell structured blends reported by Yang et al. $[57,58]$; it could be interesting for a range of applications.

\subsubsection{Filler Surface Modification}

Filler surface modification refers to changing the original inert interface on filler surface through physical or chemical approaches, thereby altering the interfacial interaction between filler and polymer matrix. Generally, the filler surface modification can reduce defects caused by original interface, enhancing the interaction between filler and matrix, promoting the dispersion of fillers in polymer matrix, thereby preventing the performance of composites from degrading. Hence, the methods of filler surface modification can be divided into chemical modification and physical modification. Chemical surface modification is the most commonly method, and it is generally used to modify the filler surface by grafting polymer chain, block polymerization, encapsulation polymerization to filler ("grafting to") [59]; group decoration or coating organic polymer on filler surface (organic surface modification) [60]; inorganic particles mainly use van der Waals force, hydrogen bonding, chemical bonding, ionic bonding and other methods to strengthen the two-phase interaction (inorganic functionalization), for which popular methods used include: deposition method, outer layer modification, mechanochemical modification, surface chemical modification and high-energy surface modification [61]. Figure 2D shows three typical methods associated with filler surface modification [62]. The purpose of organic phase interface modification in chemical method is to increase the surface polarity and roughness, adjust the surface area and crystallization performance, eliminate weak interface layer, and introduce new functional groups. Related reviews with more details can be found elsewhere $[63,64]$. Physical surface modification is usually the use of physical means such as physical adsorption, physical surface functionalization (such as ball milling and plasma treatment) to modify filler surface.

\subsection{Secondary Processing}

The secondary processing method directly affects the final structure and morphology of the composite. Both structural design and direct shaping to the composite products can be achieved during this process. In order to better understand, secondary processing technologies are categorized according to the final obtained structure and morphology as homogeneous structure, orientation structure, porous structure, layer structure, and others. The homogeneous structure contains S2 thermal/solution annealing, S7 coating, S8 film casting, S15 compression molding and S16 3D printing; the orientation structure contains S4 solid-state drawing, S5 injection molding, S6 magnetic/electric filed, S9 pre-straining, S12 two/three-roll milling calendaring and S16 3D printing; The porous structure contains $\mathrm{S} 1$ foaming/etching, S10 filtration, S11 electrospinning S13 freeze drying and S16 3D printing; The layer structure contains S3 Bi-component(co-extrusion), S14 layer by layer and S16 3D printing. S17 curing and S18 few special processing tools are not classified here because of the particularity of their use. Moreover, distinguishment of these secondary processing methods is discussed in the each $\mathrm{S}$ tool. In this section, we summarize various recent progress on processing methods according to their similarity and give an overview of their principles, classification, usage methods and scenarios.

\subsection{1 (S1) Foaming/Etching}

Polymer foam has the advantages of light weight, good heat insulation, sound absorption and shock absorption, and they have been widely used in military and civilian industries. It is commonly classified into three types [65]: closed-cell foam, partially open-cell foam and open-cell foam. Figure 3A-C corresponds to the cellular structure of polymer foams. Generally, the basic step of foam forming is to have a bubble core, which is called nucleation, then the bubble core grow or expand in polymer melt, finally diffuse out. The driving force present in forming process is gas over-saturation caused by increasing temperature or decreasing pressure. The schematic diagram of the microstructural changes foaming process with the growth of cells is shown in Fig. 3D. More details about nucleation can be found elsewhere [66]. Polymer-based foams can be prepared using physical or chemical foaming. Physical blowing agents act on polymer matrix as inert occupation. For instance, some low-boilingpoint agents act as physical blowing agent by dissolving and evaporating from polymer, such as pentane and freon [67]. Considering air pollution, environment-friendly physical 
foaming agents including carbon dioxide, nitrogen, air and water have been used for foaming. This method starts from the saturation of polymer filled with uniform concentration gas at elevated pressures, followed by heating and reducing the pressure to obtain cell nucleation. Kuang et al. reported a pressure-induced physical foaming method by supercritical carbon dioxide $\left(\mathrm{Sc}-\mathrm{CO}_{2}\right)$ to prepare "brick and mud" structure L-polylactic acid (PLLA)-MWCNTs composites [18]. The saturated $\mathrm{Sc}-\mathrm{CO}_{2}$ filled in PLLA-MWCNTs block in a high pressure at certain temperature for a restricted state, then cool down and release them to induce the cell nucleation and bubble growth to achieve uniform foaming. Chemical blowing agents take part in reaction in foaming process and giving off gas to form cell, so foam-making process is irreversible. Isocyanate and water are often used in PU foaming, azohydrazine and other nitrogen-based materials in thermoplastic and elastomeric foaming, and sodium bicarbonate in thermoplastic foaming. For example, Covavisaruch et al. employed azodicarbonamide (AC) in foaming polyvinyl chloride (PVC)/rice hull composites and investigated the effect of concentration and particle sizes of $\mathrm{AC}$ on density and cell size. With increasing foaming agent content, the cell size became smaller [68]. For industrialization, extrusion foaming and injection molding foaming technology are used for large-scale production of polymer-based composite foam [65].

During the processing of FPCs, etching is generally used to construct voids or rough surface. It is divided into dry etching and wet etching. Dry etching refers to the use of laser or plasma, generally in order to form rough surfaces and then functional modification. For example, Xu et al. used low-pressure oxygen-plasma treatment to increase the roughness of PU backbone and activate the surface of original PU foam backbones by introducing abundant oxygen-containing groups. In this work, oxygen plasma is used as an etchant to physical bombard the surface of object through insert, generate or remove functional groups [72]. Meanwhile, wet etching refers to the use of chemical solution to etch the
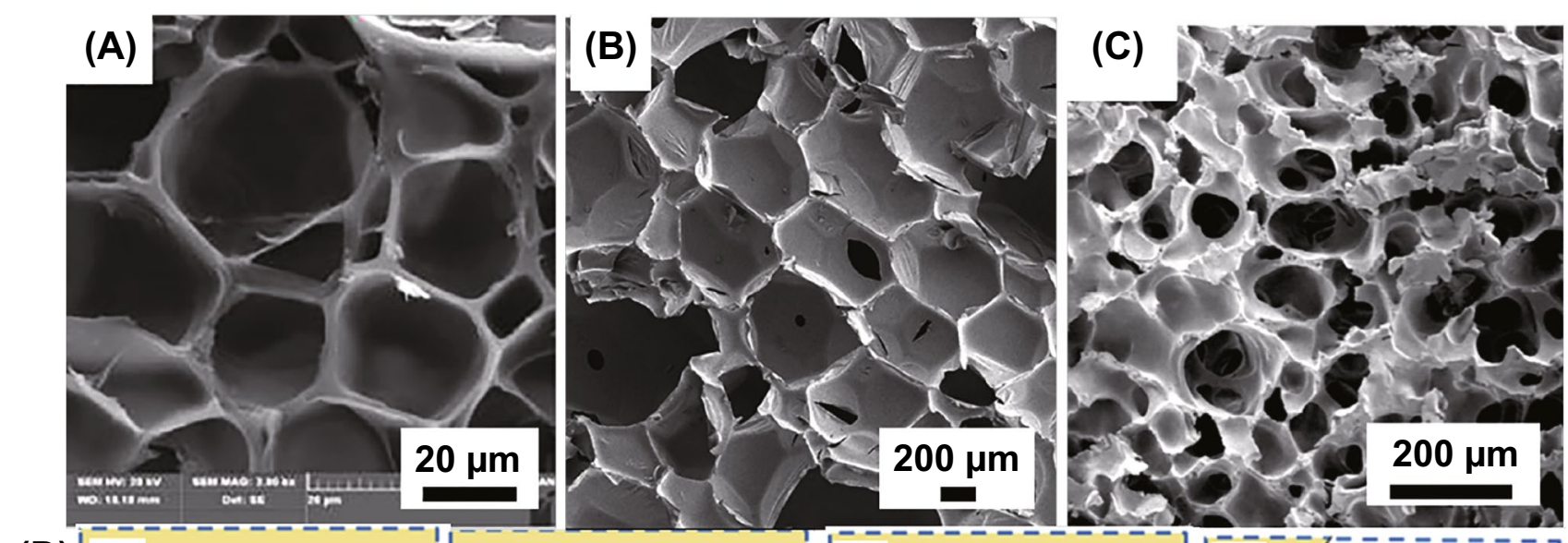

(D)
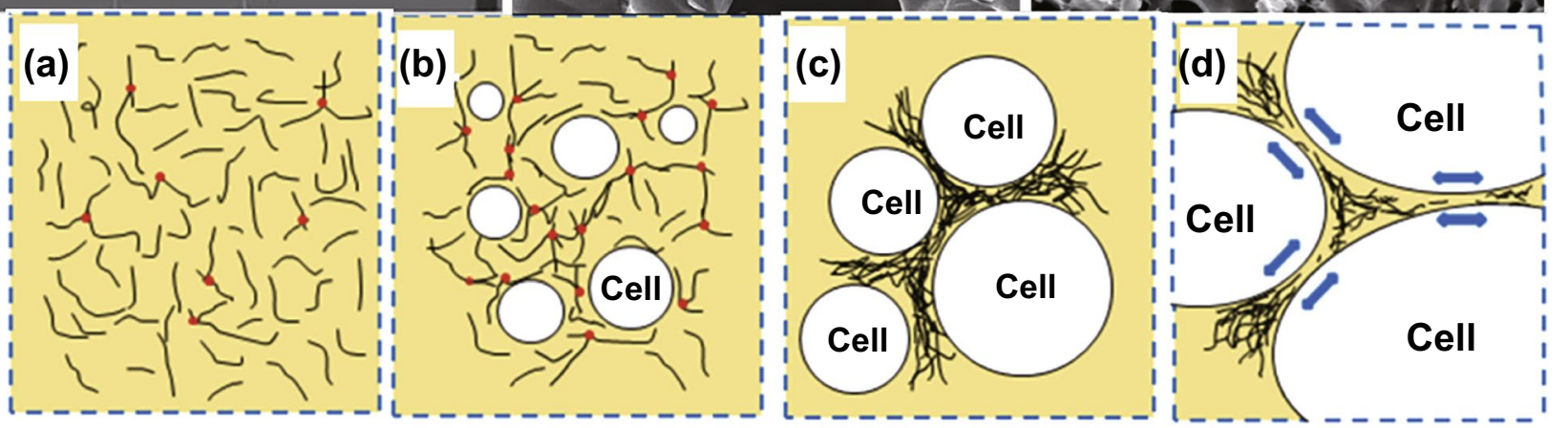

Fig. 3 Cellular structure of polymer foams: A closed-cell foam. Reprinted with permission from Ref. [69]. B Partially open-cell foam and C open-cell foam. Reprinted with permission from Refs. [18, 70]. D Schematic diagram of the microstructural changes foaming process with the growth of cells. The black lines represent the filler, and the arrows show the growth directions of the bubbles during foaming. Reprinted with permission from Ref. [71] 
interior of materials, accompanied by chemical reactions and the formation of pore structure. For example, Pang et al. grew multilayer graphene on nickel foam and formed polydimethylsiloxane (PDMS) coating by immersion graphene/Ni foam into PDMS prepolymer. Then, they employed $\mathrm{HCl}$ to etch the nickel skeleton to obtain porous structure composites, and an interconnected graphene network was obtained. This porous composite demonstrates good flexibility and can undertake bending, torsion and stretch [73].

\subsection{2 (S2) Thermal/Solution Annealing}

Thermal/solution annealing is an effective method for promoting the increase of crystallinity and morphological control in FPCs. Through such process, the polymer matrix was heated above melting temperature or glass transition temperature, accompanying the relaxation of their molecular chains. Through the relaxation and mobility of polymer molecules, the functional fillers are redistributed and the filler network structure is formed. In addition, residual stress could be eliminated. Generally speaking, the crystallinity is often increased after thermal annealing, which would often lead to changes in the mechanical properties of FPCs, such as the elastic modulus and yield strength.

In inorganic fillers/polymer systems, thermal/solution annealing could promote the formation of conductive networks in FPCs containing various types of conductive fillers. Zhang et al. showed that conductive microfibrils are separated by oriented polyethene (PE) crystals [74]. The segmental Brownian movement of PE chains facilitates the disordering of microfibrils during annealing process, in which overlapped microfibrils created new conductive paths and induced dynamic percolation transition. Furthermore, thermal annealing also has important influence on the orientation of conductive fillers. In our previous study [1], morphological control of conductive network was realized by solid-state drawing bi-component structure tape followed by annealing between the melting temperatures of different components. During annealing, highly oriented MWCNTs was relaxed with much better local contacts between still largely oriented MWCNTs bundles. In addition, another thermal/solution annealing method is sintering, where sintering temperature is usually quite high to allow the diffusion between polymer particles [75]. The influencing issues during sintering could be: sintering temperature, sintering time, pressure, particle composition, etc. Generally, thermal/ solution annealing is often combined with other technologies to achieve more effective functional design, such as electric/ magnetic field, solid-state drawing, injection molding.

\subsection{3 (S3) Bi-component (Co-extrusion)}

Bi-component (co-extrusion) is an important processing method to prepare multicomponent FPCs. In this process, polymers melt and fillers with different properties are added to the extruder and extruded with a certain shape of die under extrusion. Bi-component (co-extrusion) technology is applicable to almost all thermoplastic and some thermosetting plastics such as phenolic resin. With the advantages of simple process, energy saving, high production efficiency, greatly reduced production costs and no solvent is used in the process. Through co-extrusion, the interface between polymers produces adhesion, the adhesive force between different polymer resin composite layers mainly depends on the mutual solubility and affinity of resin matrix, and the melting temperature difference between different resins should not be very large. Additives for adhesion could be incorporated between polymer layers during co-extrusion if necessary. Meanwhile, component viscosity match, rheological behaviors, temperature control, extrusion speed and other instrument parameter design are also the key factors affecting the preparation of high performance FPCs during co-extrusion. It should be pointed out that there is a "viscosity surrounding" from low-viscosity melt to high-viscosity melt. If the die flow channel is long enough, the phenomenon of "viscosity surrounding" would lead to uneven layer thickness distribution in the final composites. Moreover, the melt with higher viscosity would be completely coated by the melt with lower viscosity. In addition, because of the different viscoelastic properties of various polymer melts, the interlayer interface of the melt is easily formed by coextrusion, producing "wavy" or "zigzag" instability.

To have more control on the multilayered structure in various polymer films, Guo et al. fabricated alternating multilayered polypropylene (PP) and carbon black (CB)-filled polypropylene ( $\mathrm{PPCB}$ ) composites through layer-multiplying co-extrusion system and investigated its electrical behaviors [76]. By controlling the co-extruding speed and the die setup, the number of layers and thickness of each layer could be controlled. These alternating multilayer composites 
exhibit anisotropic electrical behaviors because insulating PP layer would hinder the establishment of conductive pathways. Moreover, the alternating insulating PP layers and conductive layers vertical to interfaces could accumulate electrical charges at the interface, leading to significantly enhanced dielectric permittivity. More details on co-extrusion can be found in review paper by Zhang et al. and $\mathrm{Li}$ et al. [77, 78].

\subsection{4 (S4) Solid-State Drawing/Fiber Spinning}

Solid-state drawing is often carried out on materials between their glass transition and melting temperatures [79]. A suitable drawing temperature has important influence on molecular rearrangement and crystallization. As the results of drawing, modulus, strength and toughness can be improved by the strain-induced crystallization and orientation [80]. Apart from drawing temperature, draw ratio is another key factor on the mechanical properties of polymer composites.

Fiber spinning is a method to form fiber, including melt spinning, dry spinning, wet spinning, and dry-jet-wet spinning, etc. [81]. Melt spinning is one of the most widely used processes in fiber manufacturing. During melt spinning, polymers are drawn over its melt temperature thanks to the high molecular mobility of polymer melt. There is a large temperature gradient between fiber and surrounding environment. The strong shear field forces the polymer to stretch and form into filament rapidly under ambient temperature difference, and inorganic filler is often highly aligned along the spinning direction within polymer matrix. Spinning temperature, spinning rate, subsequent hot-drawing process are important factors affecting fiber performance, surface quality and crystal structure of the fibers [82]. Other factors affecting the final functional properties are the dispersion of inorganic fillers in the fiber, filler content and filler network structure, etc.

Dry spinning is a method similar to solid-state drawing and melt spinning. When the precursor polymer solution is extruded from the needle, the external evaporating hot air attacks the fiber and the solvent evaporates leaving a filamentous fiber. In the process of wet spinning, polymer composites solution is extruded and passes through a liquid precipitation bath to form fibers. And the extruded solution passes through an air route before entering a precipitation bath in dry-jet-wet spinning. Moreover, the factors affecting fiber spinning in processing are: viscosity, temperature, extrusion rate, cooling conditions (stretch ratio, precipitation conditions), etc. Overall, oriented polymer and filler network structures with different degree of alignment are often obtained during above drawing and spinning process.

\subsection{5 (S5) Injection (Blow) Molding}

During injection molding, polymer melt is stirred by a screw, injected into the mold cavity with high pressure, and then cooled and solidified to obtain product at certain temperature. In its routes, there are three main steps in the molding: filling, packing/holding and cooling [83]. Various types of polymers with desired flow properties and a low viscosity at high temperatures are suitable to produce injection molding parts, such as polystyrene (PS), polymethyl methacrylate (PMMA), PP, PE and acrylonitrile butadiene styrene (ABS). It is noteworthy that final impact on processing decision would not only depend on type of polymers but also temperature control, pressure control, injection speed and many other conditions in the process [84].

Composites exhibit a variety of microscopic morphologies under shear and temperature fields employed by injection molding, and the orientation of fillers and polymers along the injection molding direction have received widespread attention. The orientation structure of the sample is closely related to the shear field during injection molding. Due to the difference in shear force, the structure in the area close to the cavity wall (skin layer) and the center area (core layer) is significantly different [85]. For ECPCs, the composites obtained by injection molding generally have a larger percolation threshold compared to compressionmolded composites [86]. Meanwhile, more confinement on polymer composites could be achieved with decreased mold thickness and increased injection rate. For instance, $\mathrm{Yu}$ et al. observed such confinement could deform polymer blends into multilayered structure, resulting in alternating multilayer CNTs conductive networks in polyolefin blends through high-speed thin-wall injection molding [79]. Nowadays, the trend of processing technology is developing toward microinjection, high fill compound injection, waterassisted injection molding, mixed use of various special injection molding processes, foam injection molding, mold technology, simulation technology and so on [87]. 
Blow molding is a method that the polymer melt is placed in the split mold, and compressed air is introduced to make the mold embryo cling to the inner wall of the mold, which is cooled and demolded. Similar to injection molding, blow molding is also forced by shear. Compared with injection molding, blow molding products are inflated under low pressure, so the residual stress of products is smaller, and the performance in tensile, impact, bending and environmental strain resistance is often better.

\subsection{6 (S6) Electric/Magnetic Field}

Electric and magnetic fields are effective assistant method to assemble functional fillers in polymer system. Under external electric or magnetic field, filler migrates forced by electric or magnetic field, which reconfigure the filler network. For instance, alternative current (AC) electric field was used to induce nanofibrillated cellulose (NFC) alignment in TPU system in order to enhance the dielectric and mechanical properties [88]. Induced by electric field, NFC is easily orientated because of electric field-induced polarization, which induced a torque to force NFC along the electric field direction. Consequently, the prepared anisotropic TPU/NFC composites exhibit increased tensile strength and elongation at break in the parallel direction than that in the vertical direction. Magnetic field was also used to assemble filler with the participation of magnetic components, such as magnetic fibers or particles. Chen et al. [89] mixed solution of Co nanowires and polyvinylidene fluoride (PVDF) by drop casting onto aluminum sheet with magnetic field to enforce orientation, then the Co nanowires would assemble into highly ordered domains. Along the unidirectional desired direction, the electrical transport in oriented Co nanowires can be greatly promoted, and also the filler content required to form a percolated network can be possibly reduced. The results show that oriented nanocomposites exhibit significantly increased electrical conductivity than identical nanocomposites that are randomly oriented. Another strategy is that when magnetic particles adhere to the surface of filler, the magnetic particles drive the filler to orient under magnetic field. For instance, magnetic $\mathrm{Fe}_{3} \mathrm{O}_{4}$ nanoparticles on the surface of a certain type of filler could guide filler re-orientation under external magnetic field [90]. Overall, such method based on external fields often result in filler structure with some degree of orientation without significantly orienting polymer matrix. Meanwhile, better filler local contacts are also often observed.

\subsection{7 (S7) Coating: Spray/Roll-to-roll/Dip Coating/ Scraping/Spin Coating}

Coating is one of the most widely used processing methods for fabricating functional films or layer of functional coatings on various substances, such as fiber, foam, electrospun mat, film or any solid surface. Wide range of filler contents could be achieved in functional polymer composites in coating processing. According to the filler distribution in these films or any other substances, two different systems can be identified. One of them is the most adopted method: the functional filler is evenly distributed in the polymer system and then coated onto a given substance. This method has been adopted for functionalities including electrically conductivity, thermoelectric, dielectric, thermal conductivity, EMI shielding and sensors. For instance, Hong et al. [91] fabricated high-performance thermoelectric nanocomposite films as organic thermoelectric generators through spray coating a mixture of CNTs and poly(3-hexylthiophene) (P3HT). Surface modifier is often introduced into the mixed system. For instance, Shen and coworkers [92] utilized dopamine (PDA) to modify h-BN microplatelets and stirred with polyvinyl alcohol (PVA) aqueous solution, where the PDA coating increases the dispersibility of the filler and enhances its interaction with PVA matrix. Followed by scraping, the hexagonal boron nitride (h-BN)/PVA composite film was achieved.

The other is the multiple coating strategy, where separate steps were used to prepare filler and polymer layers, respectively. For example, Liang et al. presented a PDMS/Ag nanowires composite flexible transparent conductive film by spin-coating PDMS onto Ag nanowire network [93]. An Ag nanowire (AgNW) network was fabricated firstly via spin-coating of AgNW solution onto a polyethylene terephthalate (PET) substrate, and then the obtained $\mathrm{AgNW}$ network was placed on $\mathrm{HCl}$ vapor under visible light for nanowelding. Finally, PDMS layer was spin-coated onto AgNW network to obtain PDMS/ AgNW film. This strategy not only constructs controllable AgNW welding network, but also evidently enhances the conductivity. Meanwhile, dip coating is the same interface 
contact mode between filler and polymer. Kim et al. firstly dipped basalt fiber in MWCNT dispersion to prepare conductive composite filler, then the MWCNT/basalt fiber was immersed in epoxy to obtain the final MWCNT-coated basalt fiber/epoxy composites [94]. Through this method, the electrical conductivity of such MWCNT-coated basalt fibers epoxy composites increases significantly from $3.25 \times 10^{-9}$ to $1.44 \times 10^{-1} \mathrm{~S} \mathrm{~cm}^{-1}$ with increasing dip-dry coating cycle from 0 to 10 .

Overall, various coating methods have been widely used in industry to produce a variety of functional composites. These coating methods could be divided into spray coating, dip coating, comma roller coating and slot die coating. They are selected for applications according to the specific requirement and solution properties including solid content, viscosity, bubble generating ability during coating and sedimentation of solid [95].

\subsection{8 (S8) Film Casting}

Film casting is a method to prepare FPCs film by casting the mixture solution of functional filler and polymer into substrate followed by the evaporation of solvent. In this process, the mixture solution is spread out by surface tension to form a thin film. Besides, there is usually no obvious orientation structure that emerges in casting process. For instance, $\mathrm{Xu}$ et al. [96] prepared conductive PMMA/CFs (carbon fibers) composite films by casting different concentrations of CFs mixture solution on a rectangular glass plate to obtain composite films. The CFs are overlapped and disorderly distributed with each other, and finally form conductivenetwork. Compared with the above coating method, film casting tends to produce films with larger thickness, and unlike the above coating methods, it is often unsuitable for substance which is not flat films.

\subsection{9 (S9) Pre-straining}

Pre-straining is a method of pre-stretching a given object and then releasing the stress to produce special structures, such as cracks, wrinkles or bending. Through such process, functional filler network can often be oriented during stretching and relaxed during releasing. Such process has important influence on the reconstruction of conductive networks and is often used to prepare flexible and stretchable devices, such as flexible conductors and strain sensors. Wang et al. [97] fabricated a pre-straining followed buckling approach to prepare a high stretchable and sensitive CNTs film/PDMS composite with hierarchical crack structure. The PDMS solution was poured onto the multilayer CNTs film to form CNTs film/PDMS composites and cut into small strips after curing. After the strips were pre-stretched to $120 \%$ strain, network cracks were produced in multilayer CNTs film, including gaps, islands and bridges connecting separated islands (Fig. 4A). Niu and coworkers [98] stretched the PDMS substrate to $30 \%$ pre-straining and then spread a surface hydrophilic group of SWCNTs film out onto the pre-strained PDMS substrate directly. After releasing the stress, some wrinkles structure would be formed (Fig. 4B). Ge et al. [99] provided a stretchable electronic fabric artificial skin approach with a helical AgNWs network by prestraining. A $100 \%$ pre-straining elastic thread (a nylon fiber helically wound around inner PU fibers core surfaces) was coated with AgNWs solution through dip coating and then a helical AgNWs network (Fig. 4). Thanks to this unique helical structure, the AgNWs networks can still keep the conductive paths and high conductivity even if sustain large tensile strain. Besides, pre-stretching could also be used for conductive polymer composites [100,101] and thermal conductive polymer composites [102].

\subsubsection{0 (S10) Pour Casting/Filtration}

Pour casting/filtration is a typical combination method for fabricating FPCs. It is generally applicable to fillers with structural framework or fixed shape, such as 3D network structure or fillers fixed in mold. The polymer serves as protective layer and fills the gap among the fillers. For instance, Yu et al. [103] prepared high-performance epoxy nanocomposites reinforced with 3D CNT sponge for EMI shielding by filtrating epoxy into as-prepared porous CNT sponge with a vacuum-assisted method. The excellent electrical, EMI shielding and mechanical properties of epoxy/CNT sponge nanocomposites can be attributed to the more continuous and strong conductive network for electron transport, EMI shielding and load transfer. Generally, pouring casting/filtration method does not damage the structure of functional filler, $\mathrm{Gu}$ [104] reported a $\mathrm{Fe}_{3} \mathrm{O}_{4} /$ thermally annealed graphene aerogel (TAGA) EMI shielding composite by pour casting epoxy in $\mathrm{Fe}_{3} \mathrm{O}_{4} /$ TAGA aerogel. It can be observed 
that the composite remains the same filler network structure before pour casting (Fig. 5).

\subsubsection{1 (S11) Electrospinning}

Since 1990s, electrospinning has attracted a great deal of interest due to its advantage of simple operation, strong designability and yielding continuous fibers with diameters down to the nanometer scale, having a rapidly development in recent years [105]. Unlike conventional fiber spinning techniques, electrospinning is driven by electrical force, and the polymer solution overcomes surface tension to form jet under high voltage electric field, solidifies into fibers in the air and finally falls onto the receiving plate. Figure $6 \mathrm{~A}$ is a schematic showing electrospinning setup. This process is complex, and the main influencing factors include the uniform dispersion of filler, solution viscosity, applied voltage, flow rate, collecting distance, and other ambient parameters (temperature, humidity, etc.). And the fiber collected equipment is diverse, leading to various fibrous assemblies [82].

Generally, the functional filler or its precursor is uniformly dispersed in the polymer solution; thus, the filler is embedded in matrix during electrospinning process, which can make the filler realize nano-scale dispersion in matrix. In addition, electrospinning is able to prepare fibers with various morphologies: core-shell fibers (Fig. 6B) [106], beaded fibers (Fig. 6C) [107], porous fibers (Fig. 6D) [108], ribbon fibers (Fig. 6E) [109], and it is also possible to prepare oriented fibers by controlling the speed of the receiving roller (Fig. 6F) [110]. Design for functional properties is often used in electrospinning process, and Guo et al. incorporated $\mathrm{NH}_{2}$-POSS-grafted boron nitride and fluorine-containing polyimide into electrospinning fibers for excellent thermal conductivity. This method improves the interfacial compatibility and reduces interfacial thermal resistance between thermally conductive fillers and polymer matrix. And electrospinning is in favor of better contact between adjacent fillers with polymer fibers, leading to improvement in thermal conductivity [111].

\subsubsection{2 (S12) Two/Three-roll Milling Calendaring}

Two/three-roll milling calendaring is a processing method, in which by means of the strong shear force between parallel rollers, at corresponding processing temperature, viscous materials are squeezed and extended for many times. Two/ three milling calendaring can mix functional fillers and polymer matrix effectively. When the mixture pass through the gap between rotating cylinders, materials could be sheared through adjusting cylinders rotating at different velocities who impart high shear stresses. Moreover, because of the large shear stress, functional fillers and macromolecules would be oriented, which makes the film anisotropic in physical and mechanical properties. With the advantages of being solvent-free, uniform shear field, being scalable, and easy handling of high filler loadings, two/three milling calendaring has been used in various applications. For instance, Nam et al. incorporated CNT into epoxy to obtain effective electromagnetic wave absorbing composites films through three-roll milling followed laminating processes [112]. Ravindren et al. blended poly(ethylene-co-methyl acrylate)/ethylene octene copolymer (EMA/EOC) and MWCNTs by solution mixing. After drying, the composites were homogenized by passing through two roll mill subsequently [113].

\subsubsection{3 (S13) Freeze Drying}

Freeze drying is a method of drying materials using the physical phenomenon that water sublimates gas from ice [114]. After gas sublimation, hole channels are left to form porous structure. Freeze drying is essential for the creation of a dried hydrophilic substance with high specific surface area and pore volume. It is a simple and efficient approach to fabricate materials with low density and high specific surfaces due to the $3 \mathrm{D}$ highly porous structures. And as a template free preparation method, it is desirable for scalable manufacturing of aerogels with controllable density and shape.

There are various strategies to achieve freeze drying. Ordinary freeze drying usually produces disorder structure as shown in Fig. 7A [115]. Zeng et al. reported a facile freezedrying method to fabricate an anisotropic porous MWCNT/ water-borne polyurethane (WPU) composites for EMI shielding (Fig. 7B) [116]. The obtained MWCNT/WPU composites were anisotropic porous architectures interconnect by cell walls which MWCNTs interspersed into WPU matrix, forming overlapping conductive network. Moreover, freeze drying technology is also a method to rearrange fillers. Wang et al. operated a unidirectional freeze drying method by immersing 
(A) (a)
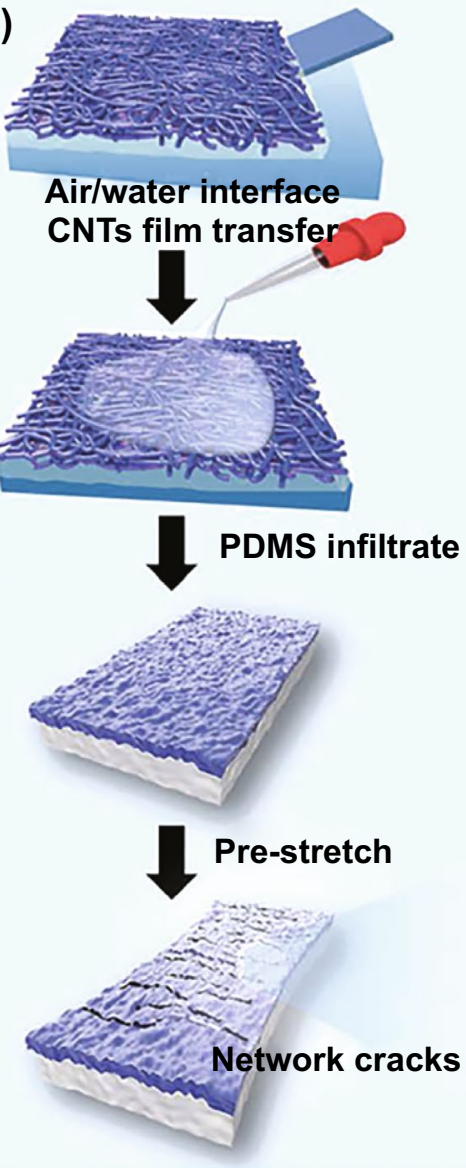

(C)
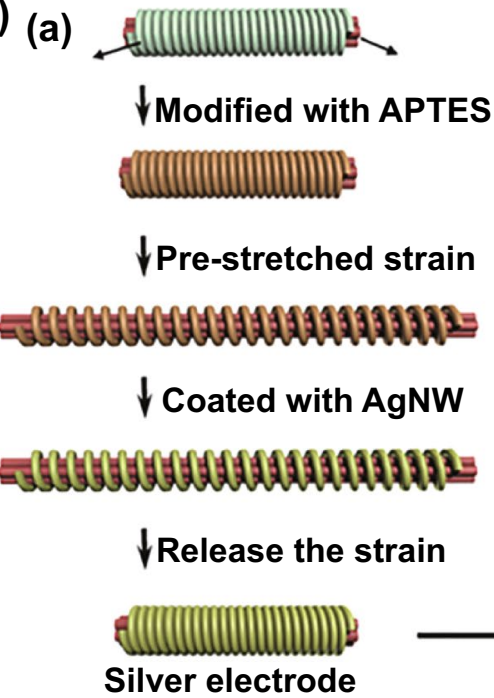
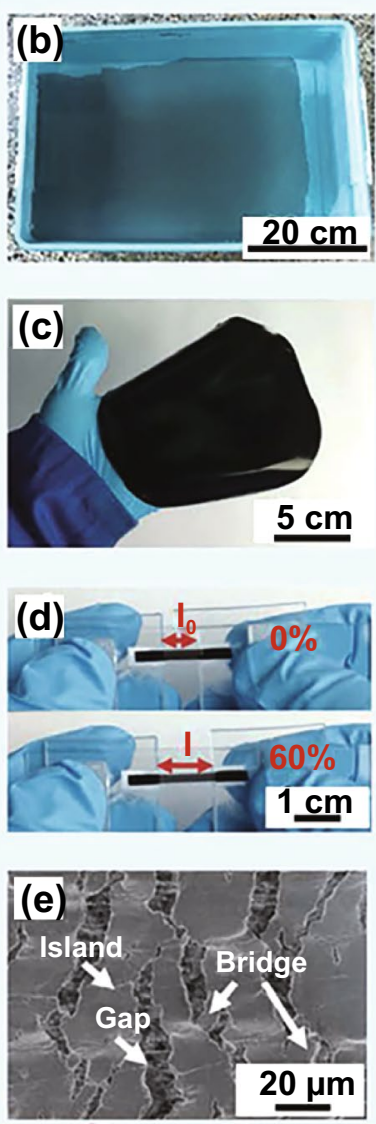

Crackes
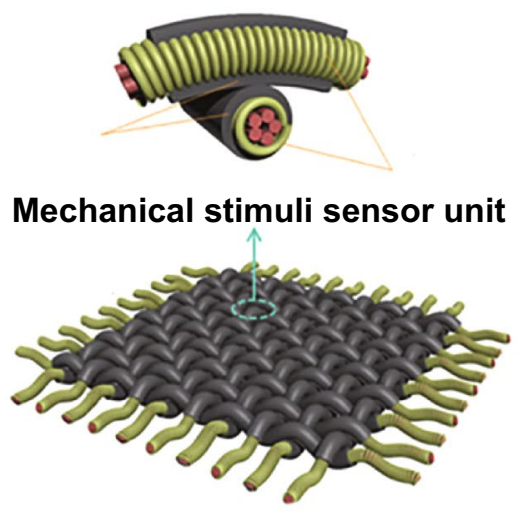

(b)

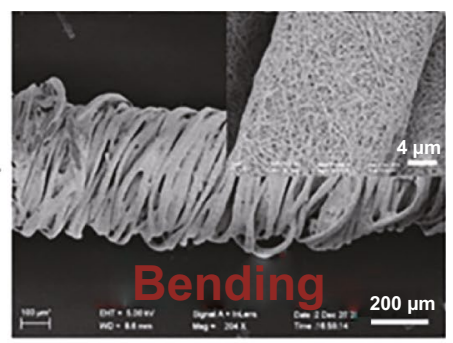

(c)

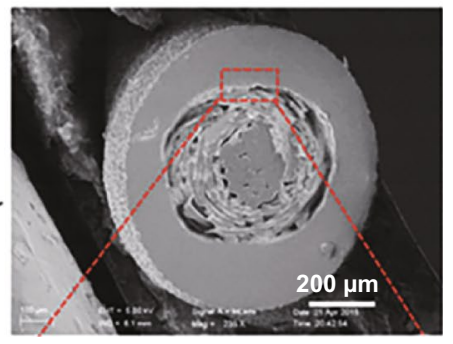

Fig. 4 A Illustration of the fabrication process of CNTs films/polydimethylsiloxane (PDMS) strain sensors and network cracks after pre-stretching, consisting of gaps, islands, and bridges connecting separated islands. Reprinted with permission from Ref. [97]. B Schematic of preparing buckled SWCNT film on PDMS and buckled structure after pre-stretching. Reprinted with permission from Ref. [98]. C Schematic illustration of the fabrication processes of the wound around polyurethane fibers core surfaces coating by AgNW with bending structure of the sensor electrode. Reprinted with permission from Ref. [99] 


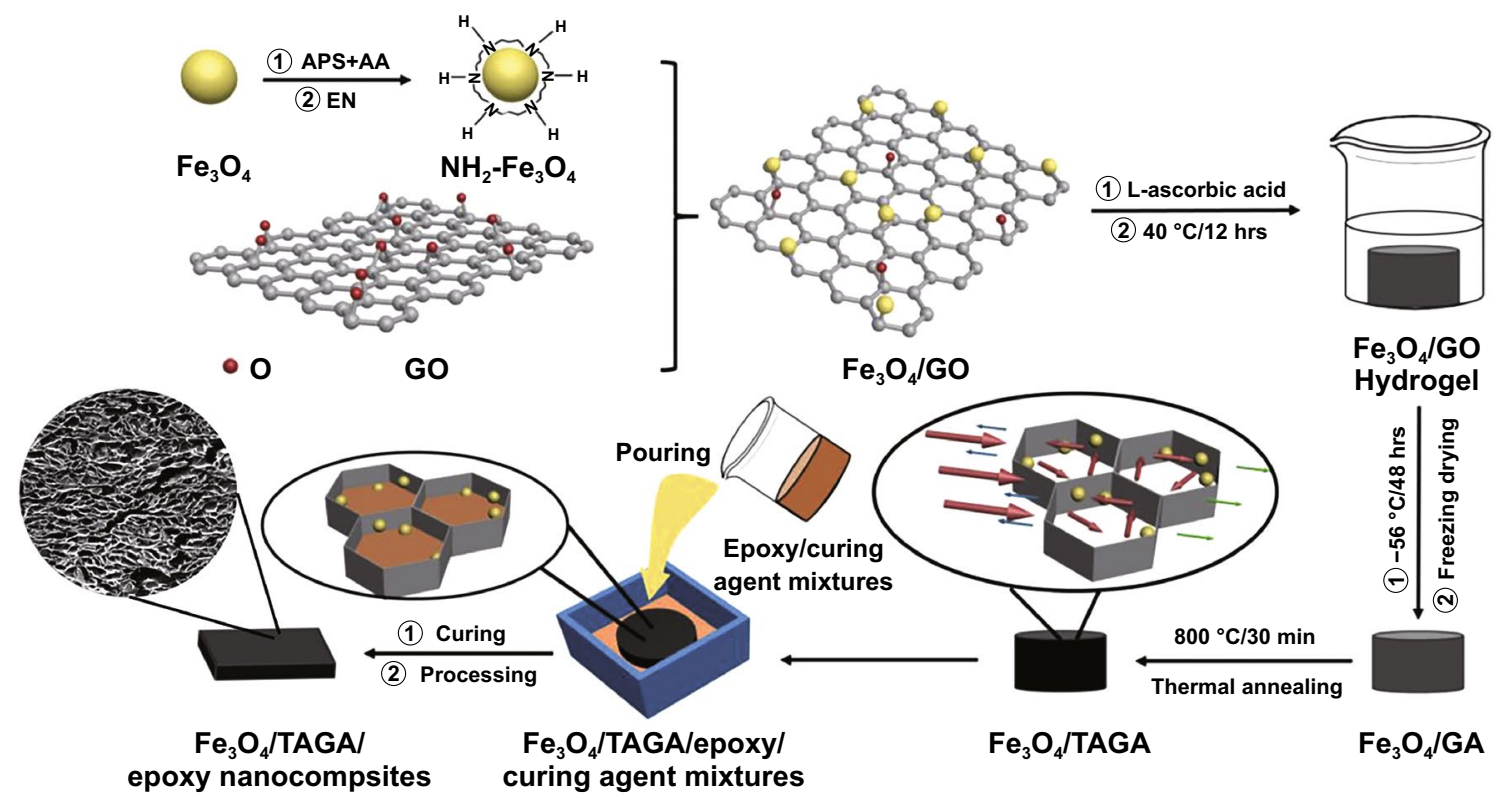

Fig. 5 Schematic diagram of the fabrication for $\mathrm{Fe}_{3} \mathrm{O}_{4} /$ thermally annealed graphene aerogel (TAGA)/epoxy nanocomposites by pour casting. Reprinted with permission from Ref. [104]

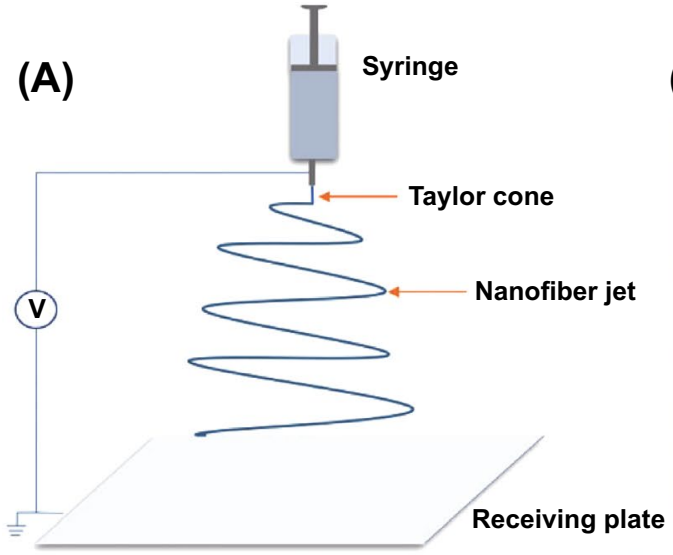

(D)

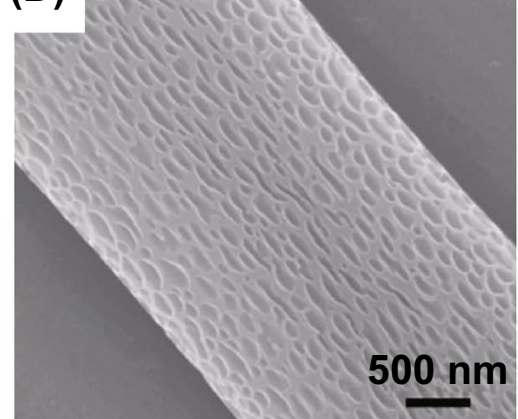

(B)

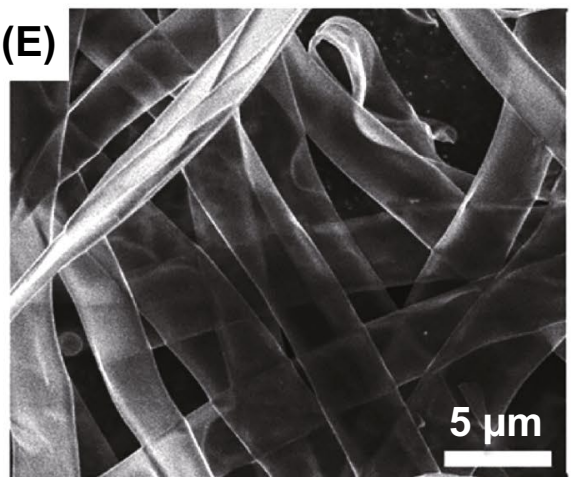

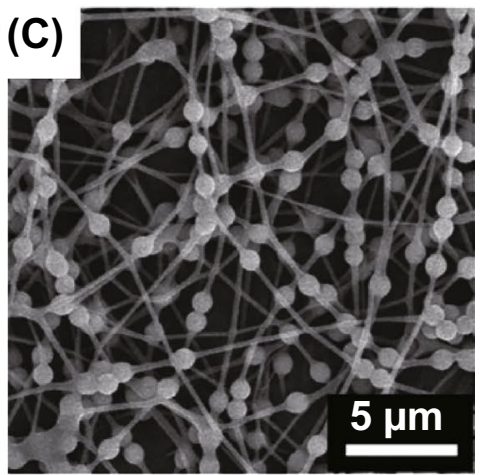

(F)

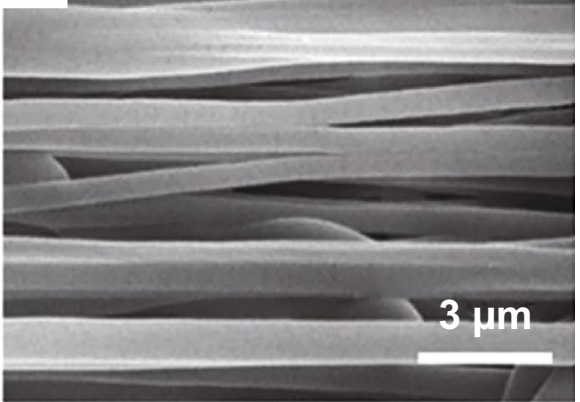

Fig. 6 Schematic showing electrospinning setup (A) and electrospinning with some special morphology: B core-shell fibers. Reprinted with permission from Ref. [106]. C Beaded fibers. Reprinted with permission from Ref. [107]. D Porous fibers. Reprinted with permission from Ref. [108]; E ribbon fibers. Reprinted with permission from Ref. [109]. F Oriented fibers. Reprinted with permission from Ref. [110] 
a precursor tube in liquid nitrogen at a constant speed with glass beads filled in tube bottom. In this system, a honeycomb-like structure with isotropic in directions perpendicular to the channels is formed (Fig. 7C), contributing excellent electrical conductivity and Young's modulus along the direction of penetrating microchannels. It is approximately twice of those in the orthogonal direction in cellulose nanofiber/ CNTs/PDMS composites strain sensors [117]. Bai and coworkers constructed 3D nacre-mimetic BN/epoxy composites conductive network by bidirectional freeze drying. They utilized a low thermally conductive polydimethylsiloxane wedge to generate temperature gradients in both the horizontal and vertical directions, guiding by which ice crystals nucleate and grow into a long-range lamellar pattern (Fig. 7D). At the same time, BN nanosheets were assembled to highly organized 3D conductive network, providing prolonged phonon pathways. Such materials illustrate a high thermal conductivity $\left(6.07 \mathrm{~W} \mathrm{~m}^{-1} \mathrm{~K}^{-1}\right)$ at $15 \mathrm{vol} \% \mathrm{BNNS}$ loading and illustrate high anisotropic thermal behavior $(\lambda l l / \lambda \perp$ as high as 12$)$, excellent electrical resistivity $\left(2 \times 10^{12} \Omega \mathrm{cm}\right)$, and thermal stability (glass transition temperature $120^{\circ} \mathrm{C}$ ) [118].

\subsubsection{4 (S14) Layer by Layer Assembly}

In the past few decades, layer by layer (LbL) assembly has received prosperous development as its simple preparation, controllable thickness and wide range of applications [78]. For the preparation of FPCs, such method is of great significance to the layer structure design and functionalities. Multilayered hierarchical structure, layer interfaces, and morphologies of multilayered polymer composites can be controlled through LbL method. The original LbL assembly technology was driven by electrostatic force as reported by Iler, where polyelectrolyte multilayers were prepared by alternate deposition in polyelectrolyte solution with opposite charges [119]. Since then, LbL assembly method has aroused great research enthusiasm. With years of continuous research, a series of LbL assembly technologies have been developed [78]: driving force by electrostatic force, hydrogen bonding, charge transfer interaction force, covalent effect, host-guest interactions, compression pressure, shearing force and temperature-field driven methods have been proposed to prepare FPCs. In addition, the LbL assem-
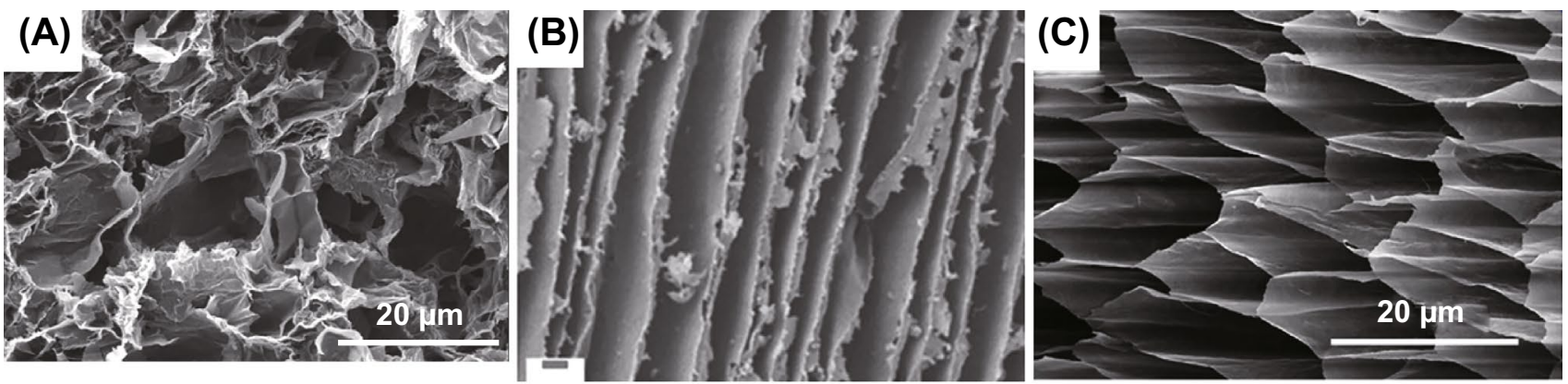

(D) BN nanosheets (BNNS)

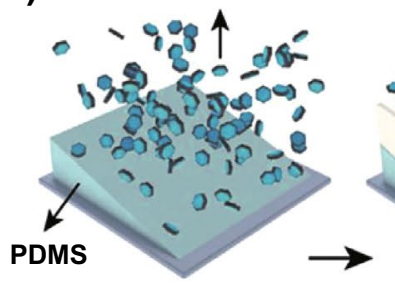

Bidirectional freezing

Lamellar structure BNNS aerogel
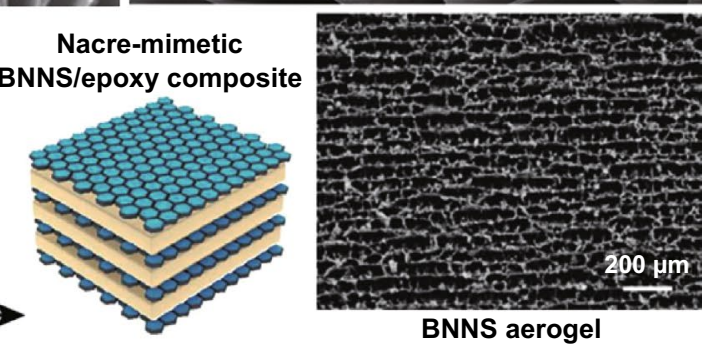

Fig. 7 A SEM of disordered freeze-dried of cellulose/graphene nanoplatelets. Reprinted with permission from Ref. [115]. B SEM of freezedried anisotropic porous MWCNT/WPU composites. Reprinted with permission from Ref. [116]. C SEM of unidirectional freeze-drying cellulose nanofiber/CNTs/PDMS composites. Reprinted with permission from Ref. [117]. D Schematic of the fabrication route using a bidirectional freezing technique to obtain BN/epoxy composites. Reprinted with permission from Ref. [118]

bly method is also expanded by strategies of immersive assembly, spin assembly, spray assembly, electromagnetic 
assembly, fluidic assembly, and so on. For example, Wang et al. [120] fabricated 3D BN nanosheet-wrapped melamine foams (MF@BNNS) by repeated LbL assembly using melamine skeleton as substrate to realize high-efficiency thermal conductivity. As polyethylenimine is positively charged, the MF foam was immersed in polyethylenimine solution for the following electrostatic interactions with negatively charged BNNSs. Then, via the alternate deposition of BNNSs and polyethylenimine multiple times by LbL assembly technique, epoxy resin was vacuum-assisting infiltrated on MF@ BNNS foam to prepare epoxy-based thermal conductive composites. Heo et al. [121] used LbL assembly method to prepare three component microwave absorbing composite of poly(acrylic acid) (PAA)/oleic acid-ferrite blend layer and a poly(allylamine hydrochloride) (PAH) layer based on electrostatic interaction. Combined with electrochemical polymerization for a high power factor, Culebras et al. [122] deposited PEDOT on as-prepared MWCNT film in a three-electrode electrochemical cell to achieve LbL assembly with a homogeneous microscopic structure. Besides, LbL-laminated assembly based on pressure, multilayered co-extrusion technology, crystallization and annealing was also applied in polymer melts processing [78].

\subsubsection{5 (S15) Compression Molding/Hot pressing}

During compression molding/hot pressing, FPCs are compressed at certain temperature under given pressure. This method is simple and highly efficient, which is intensively used in FPCs processing. In general, molding pressing/hot pressing is used as a step in combination with other processing methods [123]. Through hot pressing, the filler and polymer can form a closer interface, and finally the isolation network structure can be obtained [124]. For instance, Yang and coworker have recently reported three different methods (ball milling, freeze-drying and electrospinning) followed by hot pressing were applied to explore the thermal conductive performance based on BNNS/PVA composites films [125]. In addition to forming a filler/polymer tight interface with uniform dispersion of filler, Chen's group reported composites by solution mixing and subsequent melt pressing to afford high performance with high degree of filler orientation [126-128]. Under hot pressing, the SWCNTs with 1D nanostructure were dramatically aligned by polycarbonate (PC) melt flowing in the radial direction. This study broadens the road to obtain judicious alignment fillers in polymer composites via hot pressing [129]. Overall, processing conditions such as pressure, time and temperature are important influential issues on the structure and properties of the final FPCs, and isotropic composites are often fabricated through such process.

\subsubsection{6 (S16) Printing: Inkjet/Extrusion/Screen/3D Printing}

3D printing is a popular technology to obtain complex 3D structure devices without the typical waste in recent years [130]. Various printing techniques have been employed to fabricate polymer composites, such as fused deposition modeling (FDM), selective laser sintering (SLS), powder bed and inkjet head 3D printing (3DP), stereolithography (SLA), 3D plotting/direct-write and others new techniques are still in development [131]. Among them, direct-write and FDM technology are the most commonly ways for fabricating FPCs.

Inkjet printing/extrusion printing/screen printing are direct ink writing method of materials, which offers a promising strategy for scalable production of smart devices manufacturing with a high degree of pattern and geometry flexibility [132]. Inkjet printing/extrusion printing/screen printing refers to the process that melt or solution ink is jetting or extruded through the nozzle and drops onto substrate under surface tension, then solvent evaporate to form complex patterns. A schematic of different printing methods is shown in Fig. 8A. In this process, a challenge step is the preparation of inks, which require functional inks with suitable fluidic properties, in particular surface tension and viscosity. Many studies develop FPCs from inorganic fillers, such as CNTs, graphene, MXene and metal nanoparticle/nanowires or its alloy, and organic polymer, including epoxy, TPU, PDMS, PVA and polyvinylpyrrolidone (PVP), etc. [133]. For instance, Juntunen et al. reported an inkjet print graphene film with outstanding thermoelectric properties through dispersing graphene in isopropyl alcohol (IPA) with the aid of PVP [9]. In this system, PVP was used as dispersant and viscosity regulator to adjust the ink stability. During preparing waterbased solvent for inject printing, it is difficult to formulate and stabilize inks due to the low viscosity and weak interfacial interaction with substrate. Besides, the coffee-ring effect (the phenomenon of droplet edge gathering) would appear during solvent evaporation. Vural and coworkers employed 
MXene and proteins in aqueous solution for inkjet printing and studied its EMI shielding efficiency [134]. The binder molecule proteins can form sequence controlled assemblies with hydrogen bonding MXene crystals when high-viscosity solution of (dimethylsulfoxide (DMSO) and aqueous) and aqueous evaporates on heated substrates. Hence, the role of protein is to promote MXene dispersion and adhesion between dispersion and substrate. However, the residues of surfactants mixed in ink usually bring problems to the subsequent treatment. Zhang et al. demonstrated two types inks by MXene without additive and designed for inkjet printing and extrusion printing, as shown in Fig. 8B [135]. This additive-free MXene ink exhibits general protocols, even ohmic resistors can be inkjet-printed. Gonçalves et al. [136] reported a water-based printable piezoresistive sensors by PVA filled with MWCNT, and two printing technologies, spray printing and screen printing were used in sensor printing (Fig. 8C a-c). The spray and screen printing sensors show a similar behavior and $G F$ (Fig. $8 \mathrm{C} \mathrm{d-e}$ ) and the piezoresistive response for 10 loading-unloading cycles up to $2 \%$ strain, showing that the piezoresistive response film prepared by this ink has high universality.

As for FDM technique, the melt semi-liquid filaments are extruded layer by layer by the nozzle and then the layers are fused together and then solidify into final parts. For example, Zhang et al. printed reduced graphene oxide/polylactic acid (RGO/PLA) by dispersing RGO in PLA following melt extrusion layer by layer (Fig. 8D) [137]. This printed 3D bulk can be used as flexible circuits and printed complex structure. A number of reviews on 3D printing can be found elsewhere $[132,138]$. However, there are still some limitation in 3D printing. Firstly, the surface tension and viscosity are the key factors in making printing inks, which heavily depends on the printability and stability. Second, the use of additives (such as surfactants) makes the inks more printable in most printable inks, but how to remove these additives is an urgent issue to be solved. General follow-up processing steps such as chemical treatment or thermal annealing make it more complex for the device manufacturing process. Finally, these layer-by-layer stacking approaches can limit the geometries of devices and fine resolution. Overall, overcoming these limitations potentially could result in wide range of applications.

\subsubsection{7 (S17) Curing}

Curing is a process employed by cross-linking the functional groups on the polymer to achieve toughening or hardening, which is essential on the path to prepare thermosetting polymers and their composites [139]. In the preparation of FPCs, functional fillers are generally mixed with prepolymers, and then cross-linked polymer network is solidified and formed by curing agent so as to obtain a specific network fixed in polymer. When using the method of thermal curing, solution mixing is often used to prepare a mixed system of filler and polymer or accompanied by hot pressing or other posttreatment methods. Take the most frequently used epoxy resin as an example, no matter it is simply mixing epoxy with filler and then curing or more complex strategy through film casting-filtration-hot pressing-thermal curing, curing method is often used together with other methods [94, 140].

\subsubsection{8 (S18) Others}

Besides the processing methods summarized above, there are some others processing methods for the preparation of FPCs, such as a co-coagulation method [141, 142], flocculation method [143], solvent post-treatment [144], filler sedimentation [145], vulcanization [146], roller compression [147], steam treatment [93] and light irradiation [148]. In addition, some simple means through attachment of filler and matrix could be used to prepare conductive SSPCs [149, 150]. These methods are not universally applicable during secondary processing of FPCs, but they are still effective strategies to impact on the structure, morphology and final performance.

\section{Selection and Combination of Preparation Tools}

For practical applications, processing method is the decisive factor to determine the structure and properties of FPCs in addition to the choice of filler and matrix. Based on the above-mentioned description of different processing tools, it is not difficult to find peculiarities of T, P and $\mathrm{S}$. Thereinto, $T$-type tools represent the mixing mode of polymer matrices and fillers, most of $S$-type tools represent the approach to mold the final products, and $P$-type tools as additionally optional tools were generally utilized to control morphology 


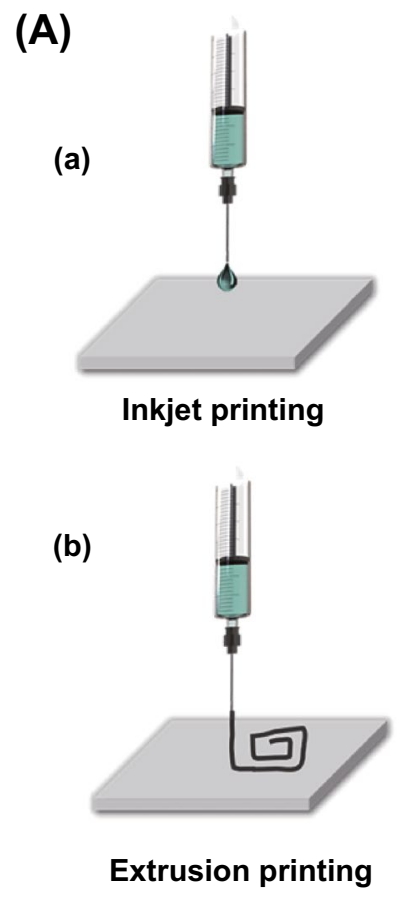

(c)

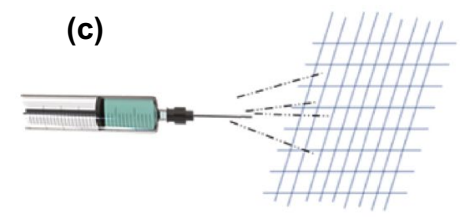

Screen or spray printing

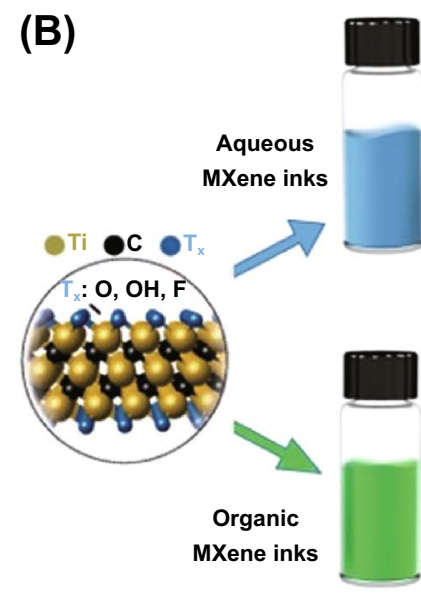

(C)

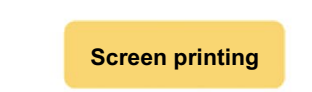

(a)

Ink.
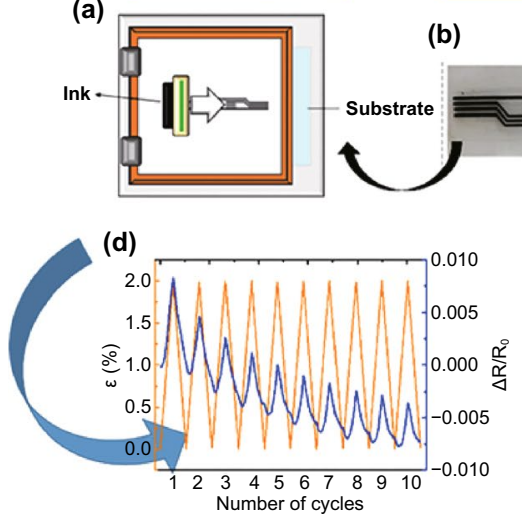

(b)

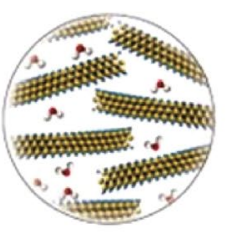

Direct MXene ink printing
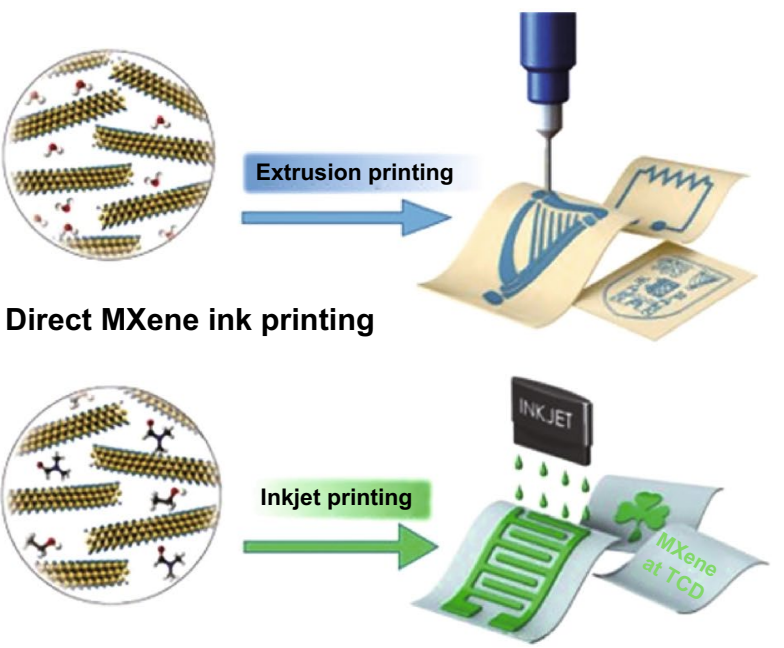

Printing technologies

Spray printing

(c)
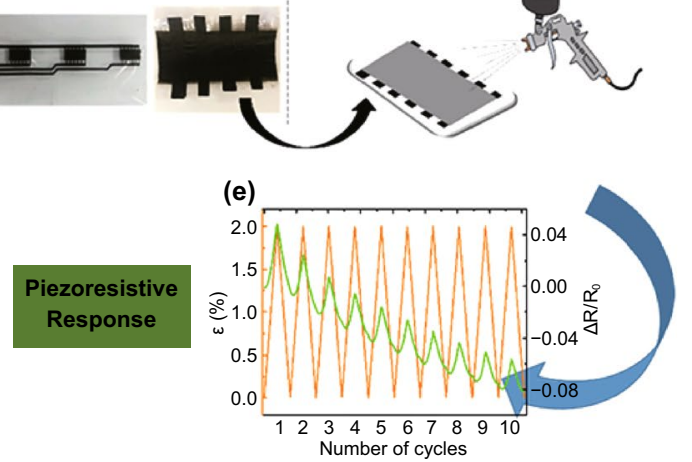

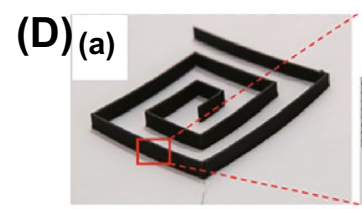

(b)

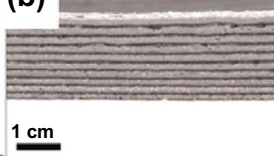

(c)

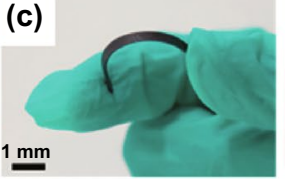

(d)

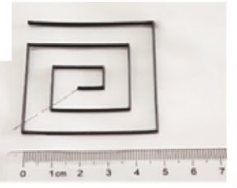

(e)

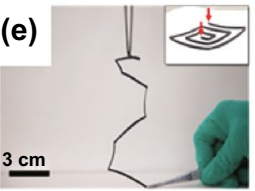

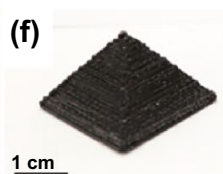

Fig. 8 A Schematic of different printings: a inkjet printing, b extrusion printing, c screen or spray printing. B Schematic illustration of extrusion and inkjet printing of additive-free MXene ink. Reprinted with permission from Ref. [135]. C Schematic of the a screen printing and $\mathbf{c}$ spray printing of PVA/MWCNT inks to prepare the piezoresistive sensor $(\mathbf{b})$. d-e show a $2 \%$ strain of the screen- and spray-printed sensors by the piezoresistive measurements for 10 loading-unloading mechanical cycles, respectively; D 3D printing of polylactic acid/reduced graphite oxide flexible circuits by melt extrusion layer by layer. Reprinted with permission from Ref. [137]

of filler network and modify the interface between polymer and filler, respectively. During the preparation of FPCs, different tool combinations are selected based on the characteristics of respective research field and the requirements of morphology and performance. We summarized 100 representative literature from recent ten years for each type of FPCs, and the selection and combination of processing tools was visualized by a diagram as shown in Fig. 9. Among these studies, it is found that one functional polymer composite was able to be fabricated by only one of processing types (namely single-type of tools), combination of any two processing types (namely dual-type of tools, containing $\mathrm{T}+\mathrm{P}, \mathrm{P}+\mathrm{S}$, or $\mathrm{T}+\mathrm{S}$ combinations) or combination of all three processing types (namely $\mathrm{T}+\mathrm{P}+\mathrm{S}$ combination). It is worth to note that non-functional polymer composites within the above-mentioned literature were prepared by only P-type 
tools, attributing to their intrinsic peculiarities. Moreover, different selection and combination of processing tools lead to the corresponding special structure within FPCs, and the selection and combination of processing tools diverse for different kinds of FPCs. The detailed description and summary were presented below.

\subsection{Single-type of Tools}

Single-type tools mean that only one processing type from the "Toolbox" is used to manufacture given FPCs. According to summarization, single-type tools often indicate only $\mathrm{S}$-type tools or merely prepared by only $T$-type tools. There is one literature wherein composites for MAPCs application with core-shell nanostructure fabricated by in situ polymerization (T2) [151]. For only $S$-type tools, common strategies to prepare FPCs are: neat polymer film, fiber, yarn or mat were firstly obtained, followed by coated, printed, injected or deposited with filler [152, 153]; or array, fabric, sponge or film of filler were fabricated at first and then combined with polymer through impregnation, infiltration, coating and attachment or LbL assembly was adopted through alternating casting, coating or filtration [13, 154].

For example, Yu and coworkers obtained wavy buckled super-aligned CNT (SACNT) films by pre-straining PDMS substrate and release (S9) to fabricate stretchable conductors as shown in Fig. 10A a [101]. This curved structure composites exhibit high stretchability and durability. For LbL assembly strategy, PVA/MXene multilayered films with excellent EMI performance were prepared through alternating multilayered casting, which exhibit a maximum EMI shielding effectiveness (SE) of $44.4 \mathrm{~dB}$ and a specific EMI $\mathrm{SE}\left(\mathrm{SSE}_{\mathrm{t}}\right.$ ) of $9343 \mathrm{~dB} \mathrm{~cm}^{2} \mathrm{~g}^{-1}$ due to the unique alternating multilayered structure and high electrical conductivity [13]. Moreover, 3D printing is another strategy to direct fabricate FPCs with only S-type tools. As shown in Fig. 10B, a conductive ink was printed into an uncured elastomeric reservoir through embedded 3D printing (S16) process [155].

In summary, single-type of tools includes only $S$-type tools that are focused on fabricating FPCs with special structure, e.g., multilayered structure, aligned filler network structure, three-dimensional interconnected filler network structure, and hierarchical structure.

\subsection{Dual-type of Tools}

Dual type of tools evidently means that two processing types were selected and combined for the preparation of FPCs, and there are three combinations: $\mathrm{T}+\mathrm{P}, \mathrm{T}+\mathrm{S}$ and $\mathrm{P}+\mathrm{S}$. It is worth noting that generally $\mathrm{T}+\mathrm{S}$ combination is the major dual type of tools to fabricate FPCs, while $\mathrm{T}+\mathrm{P}$ combination was less adopted; during the fabrication of $\mathrm{TC}$ and $\mathrm{DE}, \mathrm{T}+\mathrm{P}$ combination was not adopted and almost all of TC and DE materials were prepared by $\mathrm{T}+\mathrm{S}$ combination; three types of dual-type tools were most utilized in the fields of CPCs, MA, EMI and TE. The detail of each dual-type tools will be discussed regarding their process tools-structure-property relationship as follows.

\subsubsection{T+P Combination}

In this part, three types of structures were fabricated without $\mathrm{S}$ tools: homogenous structure, multilayered structure and core-shell nanostructure. Homogenous structure tends to be often achieved in the field of ECPCs, EMISPCs and SSPCs. For example, Han and coworkers prepared selfhealing CNT-CNF (cellulose nanofiller)/PVA electro-conductive hydrogels through solution mixing (T3) of CNT and $\mathrm{CNF}$ to obtain aqueous suspension followed by adding PVA and borax (P3), as shown in Fig. 11A [156], which illustrate a maximum electrical conductivity up to $10 \mathrm{~S} \mathrm{~cm}^{-1}$. Meanwhile, multilayered structure was obtained by in situ electrochemical polymerization (T2) of polyaniline (PANi) onto the surface of acid-treated SWCNT (P4) buckypaper, and then PANi/SWCNTs composite TE films with bilayer structure was obtained, achieving a maximum power factor of $6.5 \mu \mathrm{W} \mathrm{m}{ }^{-1} \mathrm{~K}^{-2}$ when the electrodeposited cycles at 75 [157].

Furthermore, core-shell nanostructure could be constructed for MAPCs via a similar in situ polymerization of monomer (T2) on the surface of one filler or hybrid fillers. It is worth to note that hybrid fillers and in situ polymerization of the monomer of conductive polymers, e.g., PEDOT, PANi, PPy, are preferred [16, 158, 159]. Such combination of processing tools brought out enhanced interfacial polarization which helped in improving EM absorption abilities. For instance, $\mathrm{SiC}$ whiskers-graphite nanosheet/ 

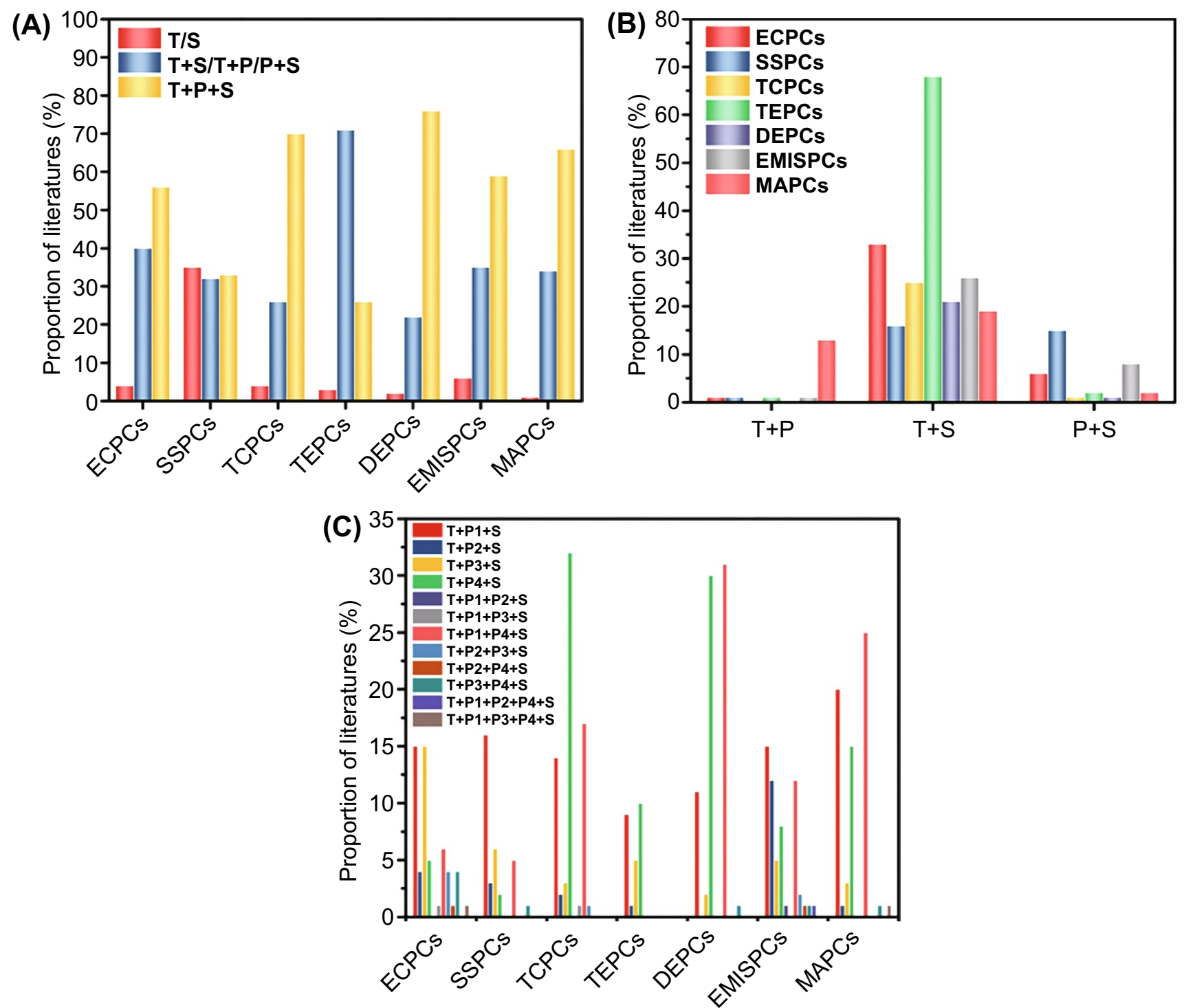

Fields of FPCs

Fig. 9 Proportion of literature for A single-type of tools, dual-type of tools, and ternary-type of tools in 100 literature of each field of FPCs, B different dual type of tools in 100 literature of each field of FPCs, C different ternary type of tools in 100 literature of each field of FPCs. Abbreviations: ECPCs (electrically conductive polymer composites); SSPCs (strain/pressure sensing polymer composites); TCPCs (thermally conductive polymer composites); TEPCs (thermoelectric polymer composites); DEPCs (dielectric polymer composites); EMISPCs (electromagnetic interference shielding polymer composites); MAPCs (microwave absorbing polymer composite)

PPy materials with core-shell nanostructure were prepared through in situ polymerization of pyrrole on the surface of hybrid SiC-graphite nanosheets (GNs) fillers (P1), resulting in that minimum reflection loss $\left(\mathrm{RL}_{\mathrm{min}}\right)$ value of $-64.2 \mathrm{~dB}$ [160] and 7.9 GHz bandwidth. Similar sandwich nanostructure also was obtained thorough in situ polymerization [161].
Generally, $\mathrm{T}+\mathrm{P}$ combination mainly includes the combination of in situ polymerization (T2), solution blending (T3) or $\mathrm{T} 2+\mathrm{T} 3$ with hybrid fillers (P1), blends (P3), filler surface modification (P4), $\mathrm{P} 1+\mathrm{P} 4$ or $\mathrm{P} 3+\mathrm{P} 4$, leading to three types of structures including homogenous structure, multilayered structure and core-shell nanostructure. 


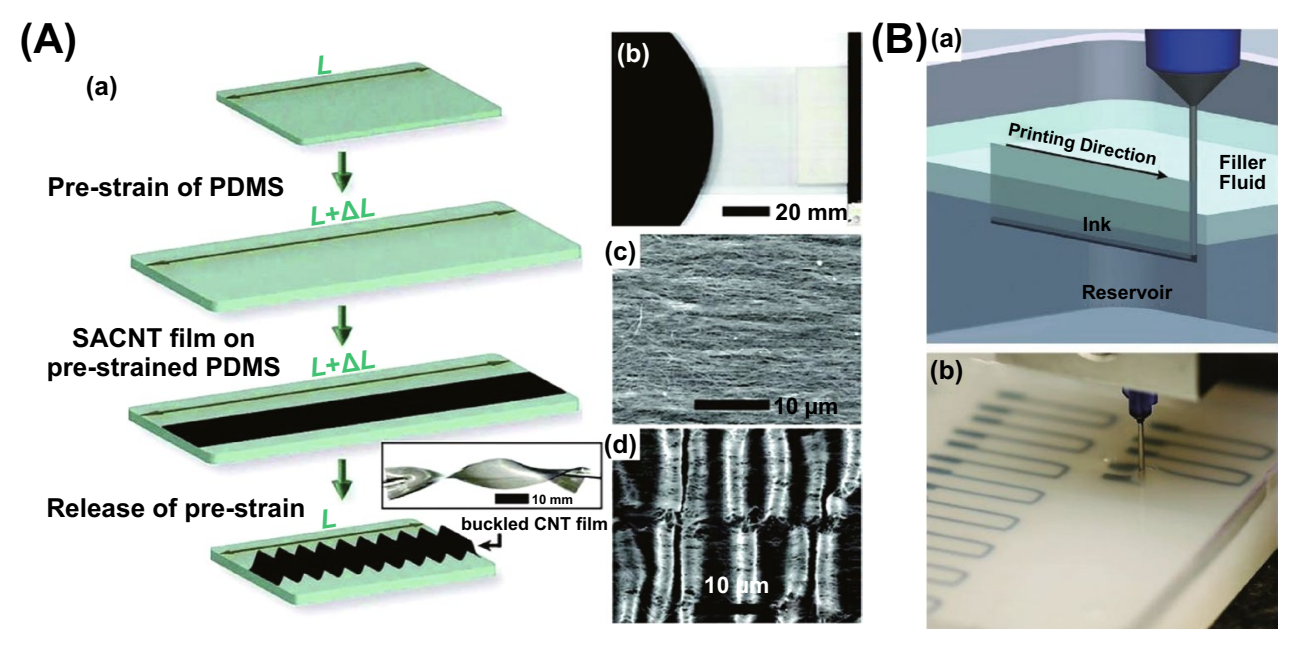

Fig. 10 A Schematic illustration for preparing polydimethylsiloxane/super-aligned carbon nanotube (PDMS/SACNT) composites films (a), the photograph of SACNT film (b), SEM images of SACNT film (c) and the buckled SACNT structures on a PDMS film after releasing pre-strain (d). B Sketch of the embedded 3D printing process with printing a conductive ink within an uncured elastomeric reservoir capped by filler fluid (a) and for preparing a planar array of strain sensors (b). Reprinted with permission from Ref. [155]

\subsubsection{T+S Combination}

$\mathrm{T}+\mathrm{S}$ combination is the frequently used dual-type tools in FPCs field, which endows these FPCs with more plentiful filler network structure, e.g., random filler dispersion structure, oriented filler network structure, and "brick-mortar" multilayered structure. Herein, $\mathrm{T} 1+\mathrm{S}, \mathrm{T} 3+\mathrm{S}$ and $\mathrm{T} 4+\mathrm{S}$ are discussed firstly as follows. Directly mixing polymer matrices and fillers to develop solid FPCs with fillers randomly dispersed in polymer components is the general and most important strategy of $\mathrm{T}+\mathrm{S}$ combination, which is involved in all seven fields of FPCs. Random filler dispersion structure is common and important structure. Meanwhile, the homogenous filler dispersion within the polymer matrix is generally the target. For example, the combination of solution blending (T3) and film casting (S8) allows the preparation of $\mathrm{PVDF} / \mathrm{Ta}_{4} \mathrm{SiTe}_{4}$ composites with $\mathrm{Ta}_{4} \mathrm{SiTe}_{4}$ whiskers homogeneously dispersed in PVDF matrix for thermoelectric application [162]. The PVDF/ $\mathrm{Ta}_{4} \mathrm{SiTe}_{4}$ composites demonstrate a maximum power factor value of $1060 \mathrm{~mW} \mathrm{~m}^{-1} \mathrm{~K}^{-2}$ at about $220 \mathrm{~K}$ in the in-plane direction.

Different from the random filler dispersion, aligned, oriented or ordered fillers network as another important filler network structure could be constructed via $\mathrm{T}+\mathrm{S}$ combination, wherein magnetic/electric field (S6) [163, 164], hot drawing/melt spinning (S4) $[165,166]$, injection molding
(S5) [167], electrospinning (S11) [6] or freeze drying (S13) [118] combined with corresponding melt blending (T1) or solution blending (T3), namely combination of T1 or T3 with S4, S6, S5, S11 or S13, that have been proved to form aligned fillers network within polymer matrix. Specifically, aligned Co nanowires network within PVDF matrix was prepared via combination of solution blending (T3), drop casting (S7) and magnetic field (S6). This contributes to obviously increase electrical conductivity in comparison with that random filler dispersion. Such composites illustrate an impressive power factor value of $523 \mu \mathrm{W} \mathrm{m}{ }^{-1} \mathrm{~K}^{-2}$ at $320 \mathrm{~K}$ [89]. In the field of ECPCs, Zhu et al. fabricated conductive PVA/GN nanocomposites based on solution blending (T3), film casting (S8) and hot drawing (S4) successively. Due to the hot drawing treatment on PVA/graphene composites, aligned graphene conductive network was observed and a superior conductivity value of $25 \mathrm{~S} \mathrm{~m}^{-1}$ at $6.25 \mathrm{wt} \%$ reduced graphene oxide with slightly oxygen content (SRGO) loading was achieved [168]. Moreover, the processing method containing solution blending (T3), electrospinning (S11) and compression (S15) could form oriented graphite nanoplatelets (GNPs) network (Fig. 11B) within PS matrix and illustrate significantly enhanced EMI performance [169]. In contrast, GNPs/PS composites with oriented GNPs network demonstrate much higher total EMI SE $\left(\mathrm{SE}_{T}\right)$ $(-33 \mathrm{~dB})$ than that of GNPs/PS composites with random 
(A) $\mathrm{T} 3+\mathrm{P} 3$

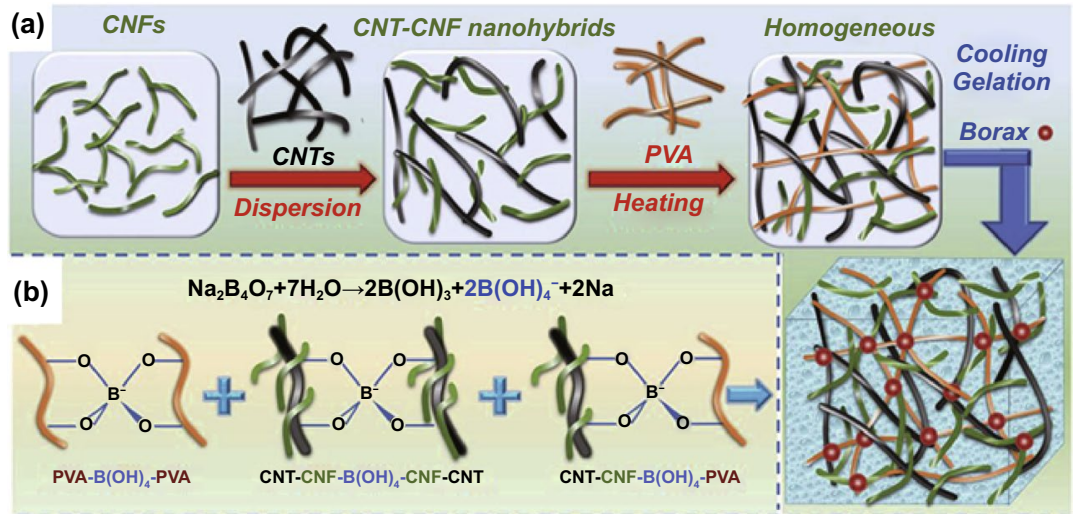

(B)

(a)

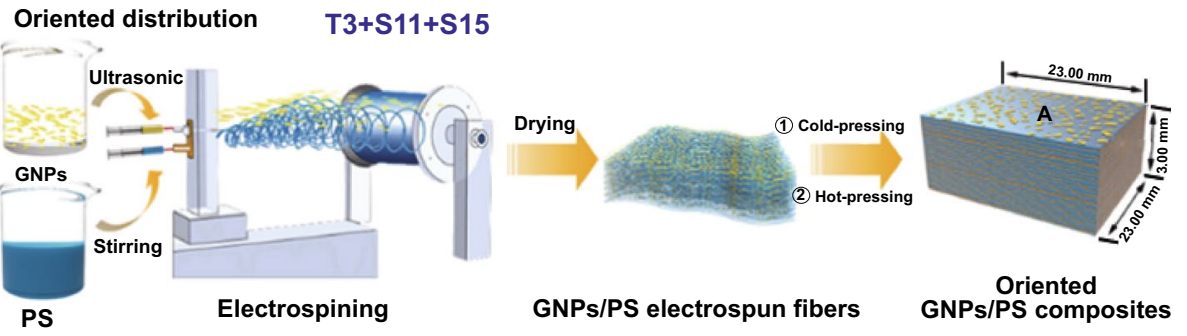

(b)
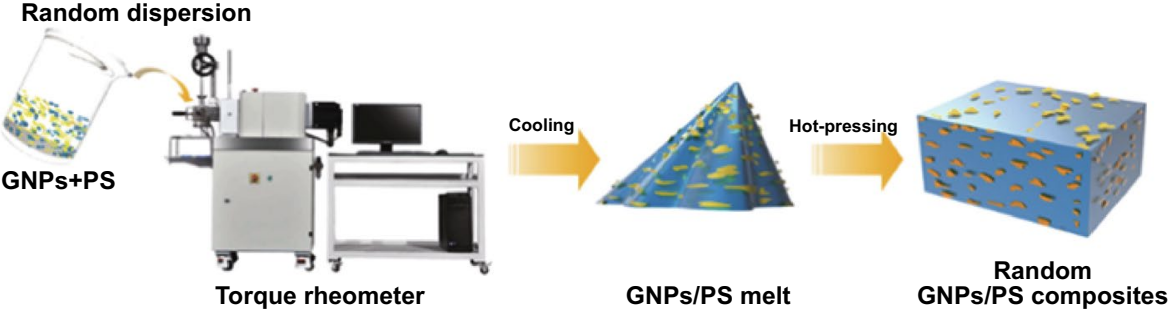

(C) $\mathrm{T} 3+\mathrm{S} 10+\mathrm{S} 13$

(a)
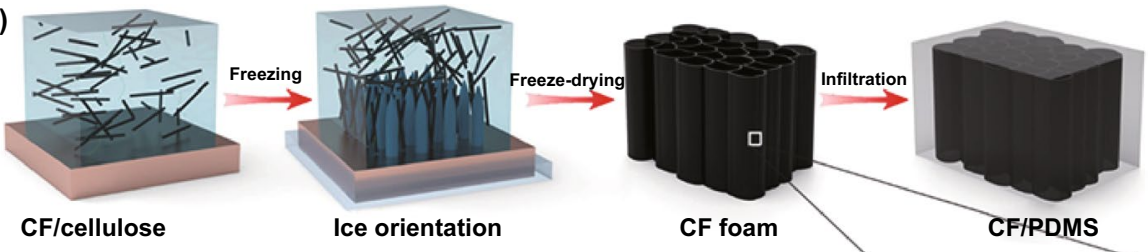

(b)
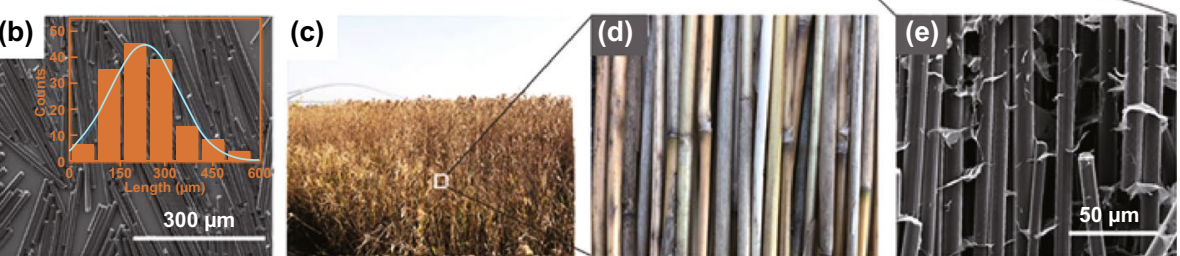

Fig. 11 A Schematic illustration of the preparation procedure of carbon nanotube/cellulose nanofiller/polyvinyl alcohol (CNT-CNF/PVA) composite gels. Reprinted with permission from Ref. [156]. B Schematic presentation of graphite nanoplatelets/polystyrene (GNPs/PS) composites with interconnection oriented GNPs network (b). Reprinted with permission from Ref. [169]; C Preparation of carbon fibers/ polydimethylsiloxane (CF/PDMS) composites to obtain vertical CF foam microstructure. Reprinted with permission from Ref. [170]

GNPs distribution $(-16 \mathrm{~dB})$ at the same GNPs content of $35 \mathrm{wt} \%$. It is worth to mention that PDMS/CFs composites were prepared as follows: solution blending cellulose and
CFs, immersing in the liquid nitrogen to freeze drying to obtain foam, then PDMS was pour casting in CFs, successively (Fig. 11C a). During freeze in liquid nitrogen, ice 
crystals randomly and rapidly grew from bottom and CFs were squeezed by ice crystals, reducing to CFs arranging in parallel with each other guided by ice crystals. Therefore, $\mathrm{CFs}$ foam with phragmites-like communis-oriented microstructure is obtained as shown in Fig. $11 \mathrm{C} \mathrm{b}-\mathrm{e}$. Owing to this special structure, the through-plane thermal conductivity of $6.04 \mathrm{~W} \mathrm{~m}^{-1} \mathrm{~K}^{-1}$ is observed at $12.8 \mathrm{vol} \%$ loading of CFs [170].

In this pattern $(\mathrm{T}+\mathrm{S})$, the use of combination of $T$-type tools and a variety of $S$-type tools to build some special network structure is a preferred choice, such as solution or melt blending followed by freeze drying (S13) or foaming (S1) or LbL assembly (S14) [171-173]. For example, Zhao et al. utilized three-step approach including solution blending (T3), compression molding (S15) and batch foaming (S1) to prepare PVDF/CNT nanocomposites with wideband microwave absorption properties [173].

For the combination of T2 and S-type tool to fabricate FPCs, it was mainly adopted in TEPCs field. For example, PEDOT/CNT thermoelectric composites with core-shell nanostructure was fabricated by in situ polymerization (T2) and vacuum filtration (S10). The strong interfacial interaction and carrier transport caused by in situ polymerization resulted in a rather high power factor of $\sim 157 \mathrm{MW} \mathrm{m}^{-1} \mathrm{~K}^{-2}$ [174]. In addition, in situ polymerization of aniline onto the surface of various fillers, e.g., SWCNTs [174], MWCNTs [175] or GO [176], could lead to ordered PANi chain structure; this ordered PANi chain structure could facilitate carrier mobility and therefore enhanced electrical conductivity, Seebeck coefficient and power factor are obtained in comparison with pure PANi.

It can be seen from the above discussion that FPCs fabricated by $\mathrm{T}+\mathrm{S}$ combination are more focused on modulating filler dispersion state within polymer matrix and interfacial interaction between filler and polymer. Meanwhile, more combination of processing tools can be adopted for preparing FPCs with optimized structure and enhanced corresponding performance due to the introduction of $T$-type tools.

\subsubsection{P+S Combination}

$\mathrm{P}+\mathrm{S}$ combination could endow FPCs with more plentiful structure originating from both $P$-type and $S$-type tools. Most of the combination of $P$-type tools and $S$-type tools includes four patterns: $\mathrm{P} 2+\mathrm{S}, \mathrm{P} 1+\mathrm{S}, \mathrm{P} 4+\mathrm{S}$ or the other combination $\mathrm{P} 1+\mathrm{P} 4+\mathrm{S}$. We will discuss examples based on the above summarization of literature in more detail as follows.

For P2 $+\mathrm{S}$, porous PLA/MWCNT composites with segregated conductive networks (P2) for EMI applications were prepared by obtaining expanded PLA (EPLA) beads after foaming PLA pellets, immersing in MWCNTs solution to wrap a layer of MWCNTs (S7) and then sintering MWCNT-wrapped EPLA to obtain PLA/MWCNTs nanocomposite foams (S2), as shown in Fig. 12A. The foams illustrate an inspiring EMI SE of $1010 \mathrm{~dB} \mathrm{~cm}^{3} \mathrm{~g}^{-1}$, owing to the unique architecture of fine microporous matrix containing conductive MWCNT networks, making it difficult for EM waves to escape [177]; For P1 $+\mathrm{S}$ in the fields of both ECPCs and EMI, interconnected spherical hollow conductive networks were constructed within silver platelets (AgPs)/RGO foam/epoxy composites by processing procedure as follows: firstly mixing GO and AgPs suspension (P1) followed by freeze-drying the mixing suspension (S13) to form a 3D framework, then thermal annealing the framework ( $\mathrm{S} 2$ tool) for the reduction of GO to obtain 3D AgPs/RGO framework, at last pouring epoxy resin into this framework (S10) and curing (S17) as demonstrated in Fig. 12B [178]; As to $\mathrm{P} 4+\mathrm{S}$, take TEPCs field as an example, PEDOT:PSS/Bi $\mathrm{Te}_{3}$ composites fabricated via treating $\mathrm{Bi}_{2} \mathrm{Te}_{3}$ particles with $\mathrm{HCl}(\mathrm{P} 4)$, followed by dip coating (S7) $\mathrm{Bi}_{2} \mathrm{Te}_{3}$ dispersion and PEDOT:PSS solution in sequence [179], wherein the treatment by $\mathrm{HCl}$ could remove the oxidized layers on the $\mathrm{Bi}_{2} \mathrm{Te}_{3}$ particles surface to achieve a more electrically conductive interface between PEDOT and $\mathrm{Bi}_{2} \mathrm{Te}_{3}$, and therefore obtaining both higher electrical conductivity and Seebeck coefficient; for $\mathrm{P} 1+\mathrm{P} 4+\mathrm{S}$, Kim et al. reported thermally conductive GO/MWCNT/epoxy composites by firstly preparing GO nanosheet and surface-modifying MWCNT with carboxylic acid functional groups (P4), then mixing GO and MWCNT suspension (P1 tool) and form a GO/MWCNT cake using vacuum-assisted filtration. It is followed by wetting the cake with epoxy resin (S10) and curing (S17). Within these GO/MWCNT/epoxy composites, MWCNTs act as interconnectors between $\mathrm{GO}$ and heat conductive bridges among the 3D microparticles due to their high aspect ratio, leading to over $140 \%$ of maximum enhancement ratio for thermal conductivity compared with GO/epoxy composites without MWCNTs [50]. In summary, the introduction 

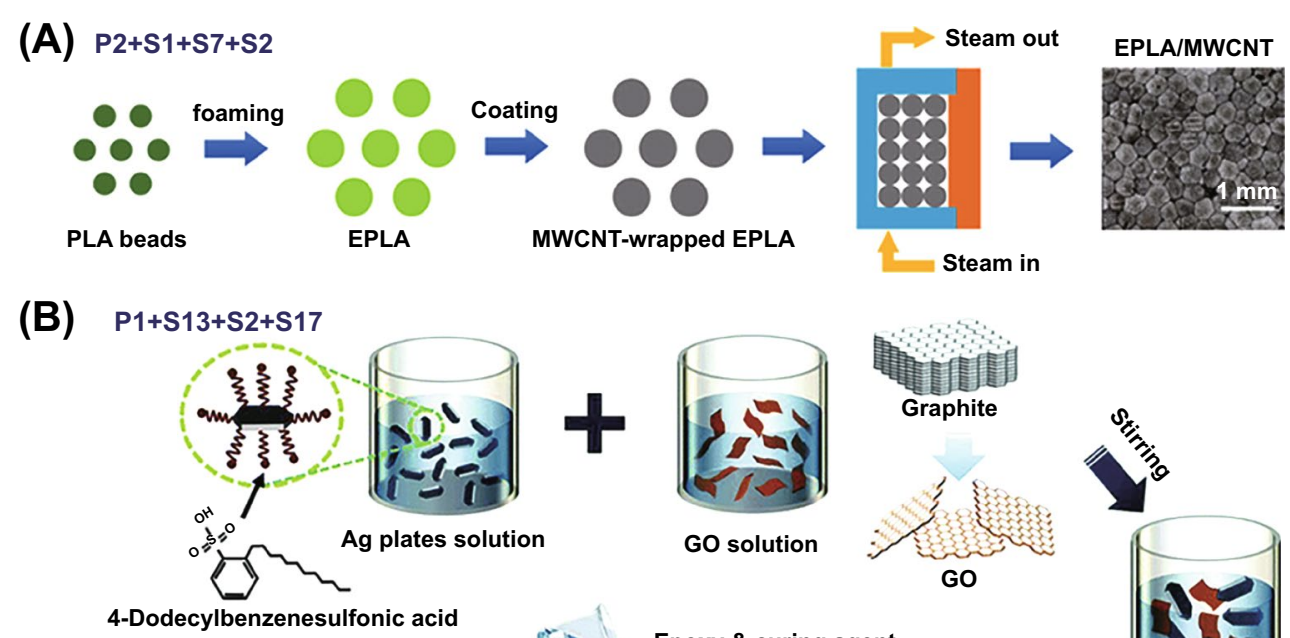

4-Dodecylbenzenesulfonic acid
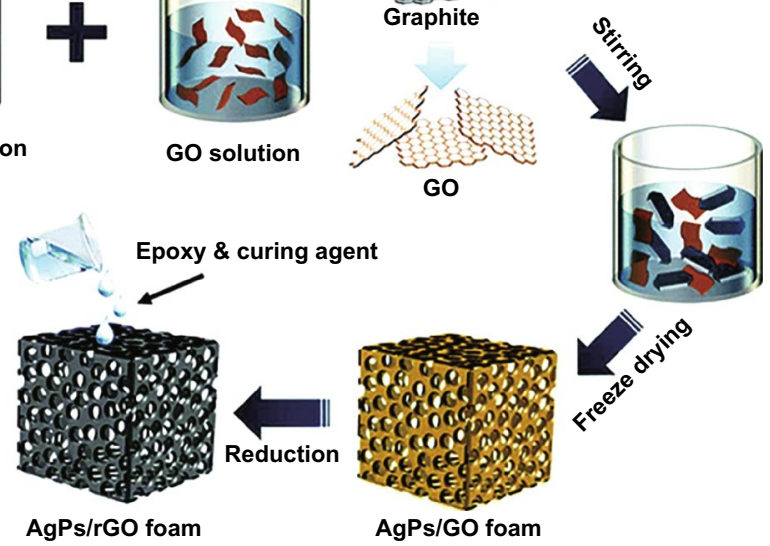

Fig. 12 A Schematic illustration of the fabrication process of polylactic acid (PLA)/MWCNT composite foam with segregated conductive CNTs network. Reprinted with permission from Ref. [177]. B Sketch of the fabrication of the AgPs/rGO foam/epoxy composite materials. Reprinted with permission from Ref. [178]

of $P$-type tools aimed at modulating filler network structure by hybrid fillers, polymer blends, volume excluding particles as well as improving interface between filler and polymer by filler surface modification. $P$-type tools are also considered to be materials selections before FPCs are processed into desired shape and structure.

\section{3 $\mathbf{T}+\mathbf{P}+\mathbf{S}$ Combination}

$\mathrm{T}+\mathrm{P}+\mathrm{S}$ combination has more multi-variant combination modes of processing tools in practice and generally more plentiful microstructures within FPCs. They are the most frequently used combinations in the literature. $S$-type tools could endow the composites with desired structure, i.e., film, foam, aerogel, multilayered structure, or some special filler network structure, e.g., oriented filler structure, segregated filler network, 3D interconnected filler network. Compared with $\mathrm{T}+\mathrm{S}$ combination, introducing hybrid fillers (P1), volume excluding particle (P2), polymer blends (P3) and filler surface modification (P4) could further improve the dispersion state of fillers within polymer matrix, strengthen the interfacial interaction between filler and polymer and modulate filler network structure. Moreover, in contrast to $\mathrm{P}+\mathrm{S}$ combination, the issue of $\mathrm{T}+\mathrm{P}+\mathrm{S}$ concerning on the dispersion state and network structure of fillers in the polymer matrix as well as the interface between filler and polymer emerges. Here, the discussion on $\mathrm{T}+\mathrm{P}+\mathrm{S}$ combination was conducted based on comparison with $\mathrm{T}+\mathrm{S}$ combination and attention was mainly paid on the $P$-type tools.

In this part, there are numerous kinds of combination of processing tools selected from "Toolbox" and the use of combination of processing tools among seven fields of FPCs is different. Comparing $\mathrm{T}+\mathrm{P} 1+\mathrm{S}$ combination $[180,181]$ with $\mathrm{T}+\mathrm{S}$ combination, $\mathrm{P} 1$ could bring out hybrid fillers network structure and synergistic effect between different fillers, which is helpful to improve the corresponding performance. For example, Voit et al. fabricated PVDF/CNTs/ $\mathrm{CB}$ composites by melt blending (T1) three components in a twin-screw microcompounder (S3) and then hot compression (S15). CNTs and CB formed string-like conductive network structures composed of CNT-CB-CNT block arrangement within PVDF matrix, therefore resulting in higher 
$\Delta R / R_{0}$ than that of composites consisting of only CNTs [182]. In the field of TE, in situ synthesis method (T2), solution mixing (T3) and drop casting (S7) were used to prepare PEDOT:PSS PC/Te/ $\mathrm{Cu}_{7} \mathrm{Te}_{4}$ (P1) composites, which demonstrate a maximum power factor of $112.3 \mu \mathrm{W} \mathrm{m}{ }^{-1} \mathrm{~K}^{-2}$ at temperature of $380 \mathrm{~K}$ (Fig. 13A). Such performance is ascribed to the synergetic effect of $\mathrm{PC} / \mathrm{Te}$ and $\mathrm{PC} / \mathrm{Cu}_{7} \mathrm{Te}_{4}$ nanorods as well as the double-carrier filtering effect at the hetero-interfaces of $\mathrm{PC} / \mathrm{Te}$ and $\mathrm{PC} / \mathrm{Cu}_{7} \mathrm{Te}_{4}$ [183].

Comparing $\mathrm{T}+\mathrm{P} 4+\mathrm{S}$ with $\mathrm{T}+\mathrm{S}$ combination, filler surface modification (P4) usually can improve the dispersion of fillers and interfacial interaction between polymers and fillers [184, 185]. For instance, epoxy/GN composites containing non-covalent functionalized non-oxidized graphene flakes were prepared by the procedures composed of non-covalent surface functionalizing graphene flakes (GFs) with pyrenebutyric acid (PBA) (P4) to obtain f-GFs, solution blending f-GFs with epoxy (T3) and curing (S17) in turn (Fig. 13B). Herein, the presence of interaction of carboxylic group with epoxy matrix enhances the interfacial adhesion and dispersion of GFs within epoxy, leading to the highest thermal conductivity of $1.53 \mathrm{~W} \mathrm{~m}^{-1} \mathrm{~K}^{-1}$ at $10 \mathrm{wt} \%$ loading of f-GFs in epoxy compared with other fillers: $\mathrm{CB}$, graphite, MWNT and GO [186].

Furthermore, in comparison with $\mathrm{T}+\mathrm{S}$ combination, introducing polymer blends (P3) is an unique strategy in this part to construct peculiar microstructures, especially in the field of CPCs, where T1 + P3 $+\mathrm{S}[187,188], \mathrm{T} 3+\mathrm{P} 3+\mathrm{S}$ $[113,189,190]$, or $\mathrm{T} 1+\mathrm{T} 3+\mathrm{P} 3+\mathrm{S}$ [191] was often used to fabricate composites with selective distributed fillers through polymer blends with different morphologies. For example, Bizhani and coworkers reported PC/PS-co-acrylonitrile (SAN)/MWCNTs composites fabricated by $\mathrm{T} 1+\mathrm{P} 3+\mathrm{S} 15$ strategy: first melt blending PC and MWCNTs and then melting blending PC/MWCNTs composites with SAN followed by hot compressing (Fig. 14A) [192]. In PC/SAN/ MWCNTs composites, PS/SAN illustrates co-continuous morphology, and MWCNTs are preferentially localized in PC phase, leading to an electrical double percolation threshold around $0.32 \mathrm{wt} \%$ of MWCNTs and a maximum EMI SE value of $-25 \sim-29 \mathrm{~dB}$ containing only $1 \mathrm{wt} \%$ MWCNTs at a thickness of $10 \mathrm{~mm}$ due to the special conductive pathway. Similar co-continuous morphologies of polymer blends were also constructed in other studies to modulate conductive networks [193]. Besides co-continuous morphologies, other morphologies of polymer blends, e.g., polymer blends with tri-continuous morphology [57], immiscible polymer blends [194] were also adopted to control the filler network.

Moreover, as shown in Fig. 9C, $\mathrm{T}+\mathrm{P} 4+\mathrm{S}$ was preferred in the field of TCPCs and DEPCs in comparison with other fields. This indicates that filler surface modification was more frequently used to prepare TCPCs and DEPCs. For TCPCs, filler surface modification involved diverse methods, such as grafting [195], group decoration [196], inorganic functionalization [197] and organic surface modification [92]. In DEPCs field, filler surface modification was mainly applied to form core-shell structure with filler as core and polymer as shell to homogeneously disperse fillers within polymer and strengthen the interfacial interaction. For TCPCs, homogeneous dispersion of fillers and enhanced interfacial interaction between filler and polymer lead to reduced thermal resistance and phonon scattering and thus improve thermal conductivity, while for DEPCs, homogeneous distribution of filler leads to reduced concentration of electric field in the polymer matrix; thus, breakdown strength can be enhanced. Meanwhile, good dispersion of filler and enhanced interfacial interaction between filler and polymer result in high dielectric constant and low dielectric loss [198].

$\mathrm{P} 2$ is a special primary processing tool to obtain segregated network structure that almost only emerges in $\mathrm{T}+\mathrm{P}+\mathrm{S}$ combination. For example, in the field of SSPCs, stretchable strain sensors with robust segregated network structure were prepared by Sang et al. based on TPU and carbon nanostructures (CNS) via grinding mixing TPU power and CNS (T3), followed by directly compression molding (S15) after drying (Fig. 14B a-d). In such composites, TPU phase acts as polymer matrix as well as volume excluding particle (P2) and CNS trapped at the interfaces of TPU powers, forming interconnected conductive networks throughout the composites, as demonstrated in Fig. 14B e-g. These strain sensors demonstrate a high gauge factor of 6861 at strain $\varepsilon=660 \%$ with $0.7 \mathrm{wt} \%$ filler and a few orders of magnitude higher electrical conductivity, much higher elongation at break than that of TPU/CNS composites at the same filler content fabricated by melt mixing [199]. Besides, the combinations of $\mathrm{T} 2+\mathrm{P} 2+\mathrm{S}$ or $\mathrm{T} 3+\mathrm{P} 2+\mathrm{S}$ could also be applied to construct segregated network structure [200].

In contrast to other combination of $\mathrm{T}+\mathrm{P}+\mathrm{S}$ containing two kinds of $\mathrm{T}$-type tools, $\mathrm{T}+\mathrm{P} 1+\mathrm{P} 4+\mathrm{S}$ was more 
(A)

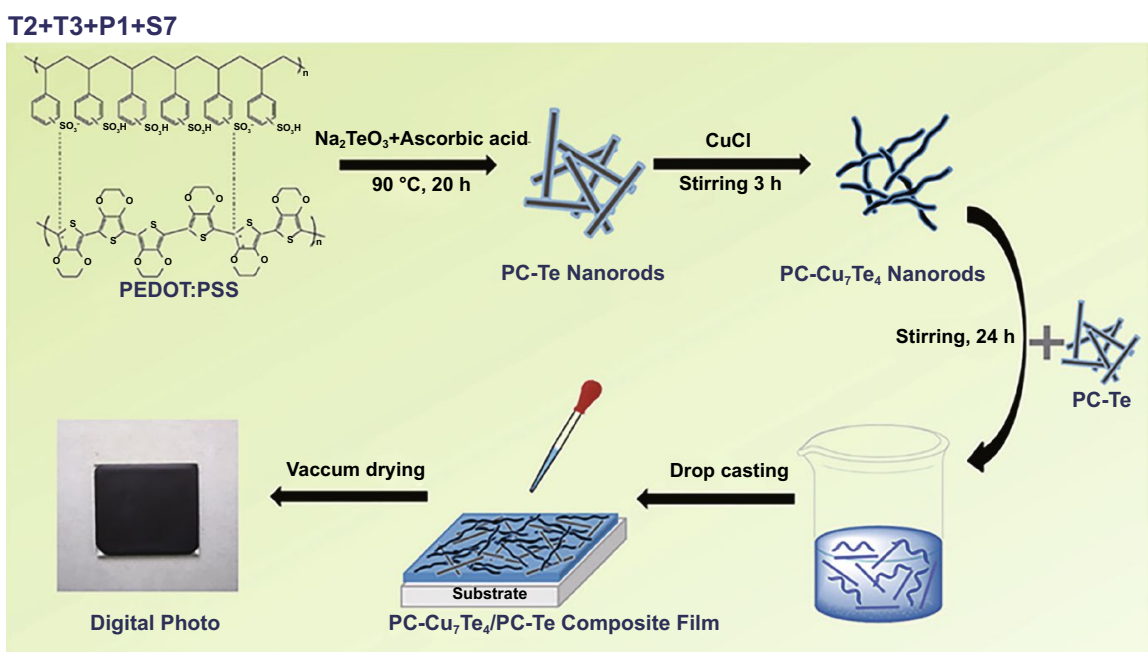

(B) $\quad \mathrm{T} 3+\mathrm{P} 4+\mathrm{S} 17$

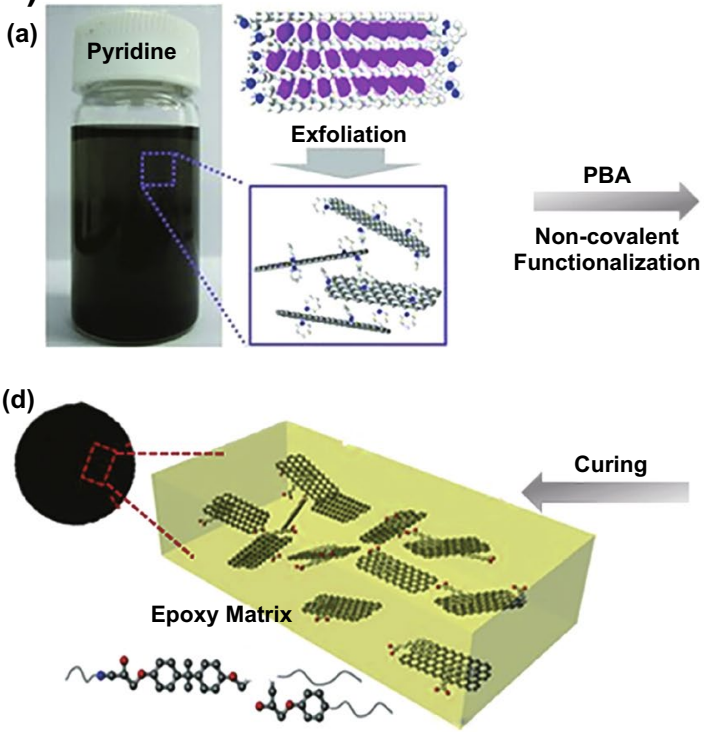

(b)
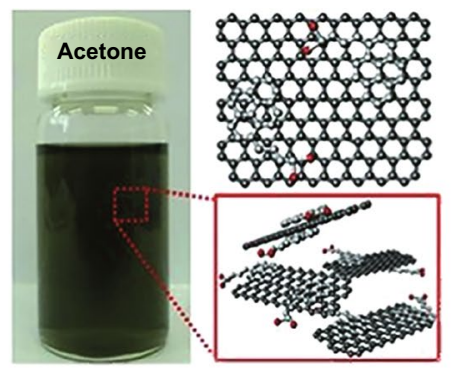

(c)
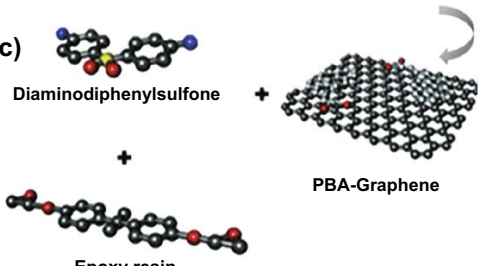

PBA-Graphene

Epoxy resin

Carbon Ooxygen Nitrogen OSulfur

Fig. 13 A Schematic illustration of the fabrication process for polycarbonate (PC)/Te/Cu $\mathrm{Te}_{4}$ thermoelectric composites. Reprinted with permission from Ref. [183]. B Schematic plot of the fabrication for epoxy/f-GFs composites. Reprinted with permission from Ref. [186]

preferred for fabricating DEPCs and MAPCs. For $\mathrm{T}+\mathrm{P}+\mathrm{S}$ combination containing $\mathrm{P} 1+\mathrm{P} 4$, a strategy containing fabricating core-shell hybrid fillers and then blending with polymer was a vital processing method to obtain composites with excellent dielectric performance [201], wherein most of the core-shell hybrid fillers had insulting shells, e.g., $\mathrm{Al}_{2} \mathrm{O}_{3}$ shells, $\mathrm{ZnO}$ shells, GO shells [202, 203], and these insulting shells could suppress formation of conductive pathways and carrier migration and therefore reduce the leakage current as well as dielectric loss. For example, PVDF/core-shell $\mathrm{CaCu}_{3} \mathrm{Ti}_{4} \mathrm{O}_{12} @ \mathrm{Al}_{2} \mathrm{O}_{3}\left(\mathrm{CCTO} @ \mathrm{Al}_{2} \mathrm{O}_{3}\right.$ ) nanofibers composites was fabricated via the following procedure: (i) core-shell CCTO@ $\mathrm{Al}_{2} \mathrm{O}_{3}$ nanofibers (P1, P4) were fabricated via coaxial electrospinning and then hightemperature calcination; (ii) $\mathrm{CCTO} @ \mathrm{Al}_{2} \mathrm{O}_{3}$ nanofibers were surface modified with PDA (T4); (iii) PVDF/CCTO@ $\mathrm{Al}_{2} \mathrm{O}_{3}$ nanofibers composites were prepared by solution blending modified nanofibers with PVDF (T3), film casting (S8) and thermal annealing (S2) [204]. The insulating layer of $\mathrm{Al}_{2} \mathrm{O}_{3}$ could reduce the charge accumulation and thus decrease the leakage current density and dielectric loss. In addition, $\mathrm{Al}_{2} \mathrm{O}_{3}$ layer also acts as buffer layer and reduces the 
(A)
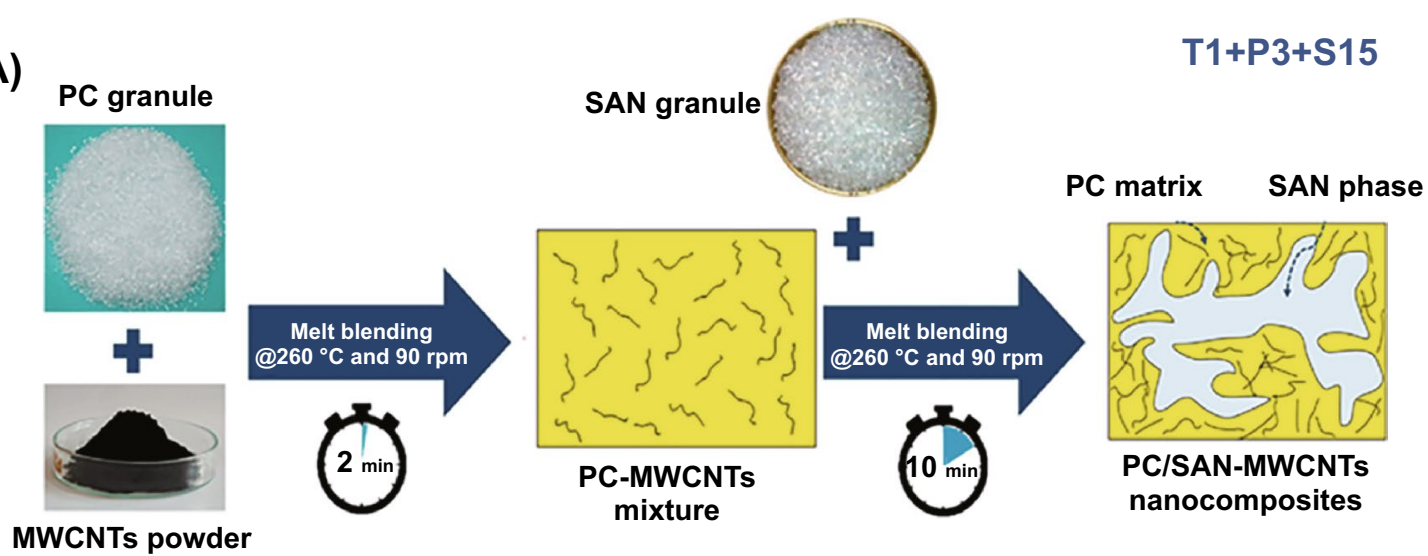

(B)

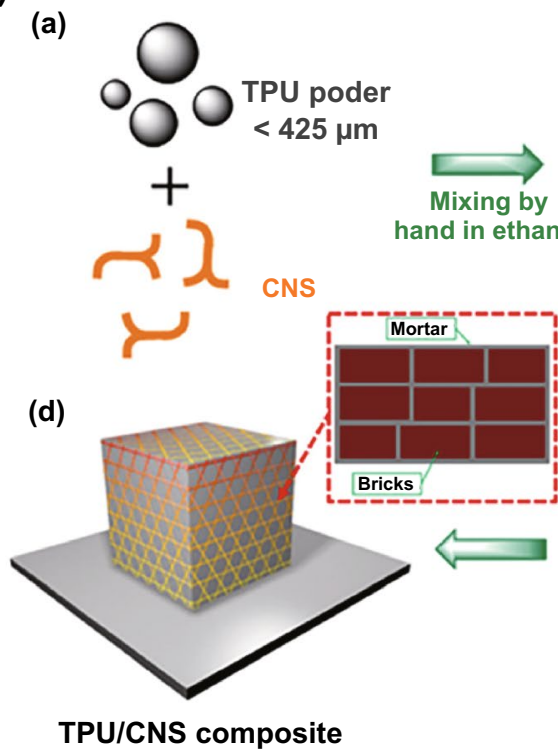

(b)

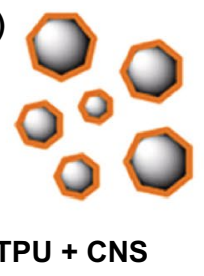

(c)

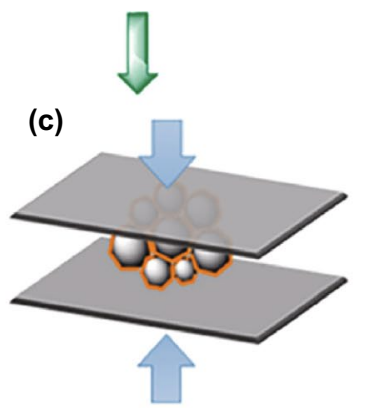

Compression molding

$\mathrm{T} 3+\mathrm{P} 2+\mathrm{S} 15$
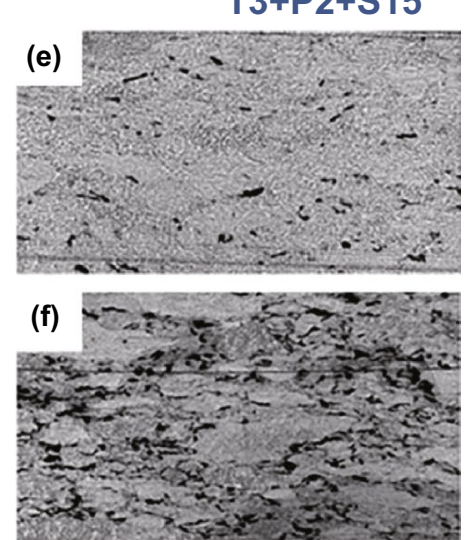

(g)

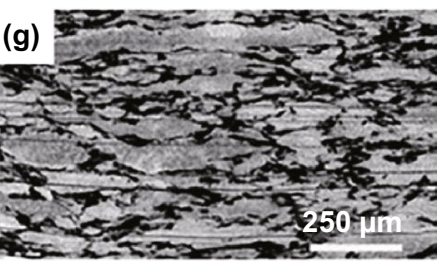

(C)

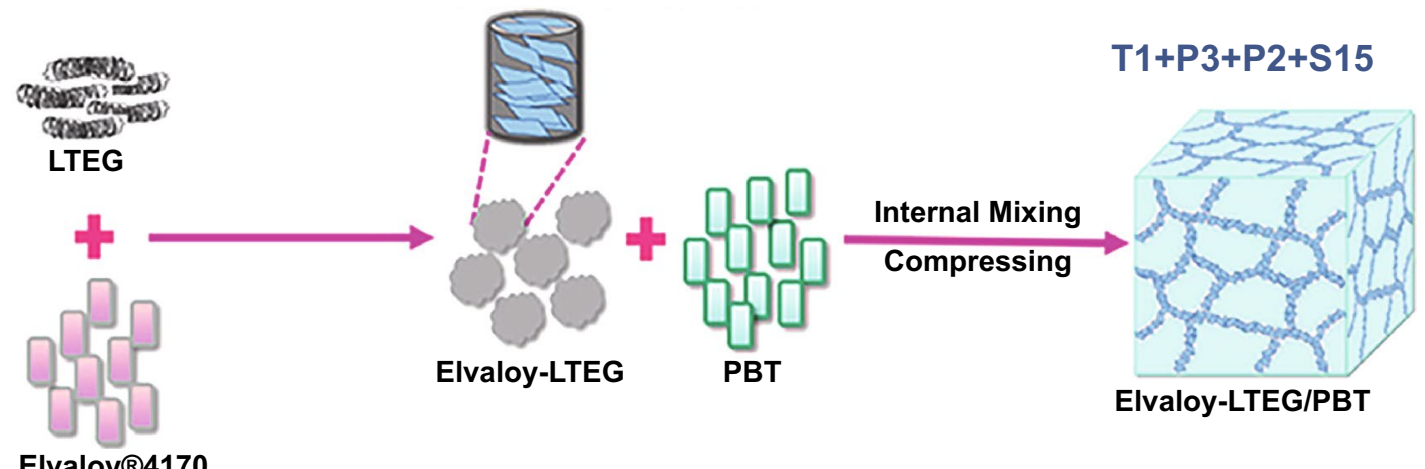

Fig. 14 A Schematic plot of the fabrication for PC/SAN(polycarbonate/PS-co-acrylonitrile)/MWCNTs composites. Reprinted with permission from Ref. [192]. B Sketch of the preparation procedure and characterization of thermoplastic polyurethane carbon nanostructures (TPU/CNS) composites with segregated CNS network structure. Reprinted with permission from Ref. [199]. C Schematic of the preparation procedure for Elvaloy@ 4170 low-temperature expandable graphite/poly(butylene terephthalate) (LTEG/PBT) composites with segregated network. Reprinted with permission from Ref. [212] 
dielectric constant difference between PVDF and CCTO, resulting in a higher electric breakdown strength in comparison with that of PVDF/CCTO composites at the same filler content. Apart from the insulating layer, surface decoration of $\mathrm{Ag}$ [205], $\mathrm{Fe}_{3} \mathrm{O}_{4}$ [206] was also used to fabricate core-shell hybrid fillers.

Moreover, there are still many studies wherein similar processing methods or others containing $\mathrm{T}+\mathrm{P} 1+\mathrm{P} 4+\mathrm{S}$ combination were used to fabricate other types of FPCs [207, 208]. Additionally, hybrid fillers (P1) or filler surface modification (P4) was introduced to combine with $\mathrm{T}+\mathrm{P} 3$ for the preparation of FPCs [209, 210]. The combination of polymer blends (P3) and volume excluding particle (P2) is another effective strategy to fabricate FPCs with segregated filler network [211]. For instance, our group added low-temperature expandable graphite (LTEG) into a commercial impact modifier (Elvaloy4170) and then coated onto poly(butylene terephthalate) (PBT) particles to fabricate PBT/Elvaloy4170/ LTEG thermally conductive composites by combining twostep internal mixing (T1) at preset temperature and hot compression (S15) (Fig. 14C). In such composites, a continuous segregated filler network is constructed by continuous Elvaloy4170-LTEG phase and segregated PBT particles phase (equivalent to volume excluding particles (P2, P3). These composites exhibit a maximum thermal conductivity up to $17.8 \mathrm{~W} \mathrm{~m}^{-1} \mathrm{~K}^{-1}$, which was significantly prior to that of composites made by directly melt mixing three components at the same filler content. Meanwhile, significant effect of PBT particles size, namely segregated phase size, on the thermal conductivity of PBT/Elvaloy4170/LTEG was also reported [212]. Besides, some more complicated combinations, such as $\mathrm{T}+\mathrm{P} 1+\mathrm{P} 2+\mathrm{P} 4+\mathrm{S}$ or $\mathrm{T}+\mathrm{P} 1+\mathrm{P} 3+\mathrm{P} 4+\mathrm{S}$, were also applied to prepare ECPCs, EMISPCs or MAPCs. In the field of ECPCs, solution blending (T3), hybrid fillers (P1), blends (P3), filler surface modification (P4), freeze drying (S13) and hot pressing (S15) were applied for preparing styrene-butadiene rubber (SBR)/PVP/RGO/CNT composites, wherein $\mathrm{PVP}$ acts as dispersing agent to improve the dispersibility of RGO and CNTs hybrid fillers. Due to the sandwich structure of RGO-CNTs hybrid fillers, restacking of RGO and agglomeration of CNTs were prevented, and thus, a high electrical conductivity was obtained compared with SBR/PVP/CNT at the same filler content [213]. This $\mathrm{T}+\mathrm{P}+\mathrm{S}$ combination with three kinds of P-type tools was merely adopted to fabricate FPCs, which is attributed the tedious processing procedure and relative high cost.
In conclusion, there are numerous selections of processing tool for fabricating FPCs. However, the selection and combination of processing tools varies obviously among seven fields of FPCs as discussed above. On the one hand, the combination of processing tools for seven types of FPCs depends on different mechanisms underlying the structure-property relationship in each field; on the other hand, owing to similarities, e.g., containing polymer matrix and filler, involving filler network and interfacial interaction, different fields of FPCs could learn from each other.

\section{Summary and Future Prospects}

FPCs are composed of fillers and polymer matrices, while the various functionalities and properties of different composites can vary greatly. In the past decade, a variety of FPCs have been synthesized and explosive progress has been achieved for different functionalities, including electrical conductivity, strain sensing ability, thermal conductivity, dielectric properties, electromagnetic shielding (EMI), microwave absorption and thermoelectric property. These functional properties are largely determined by filler, polymer, network morphology and interfacial interaction. All of these could be considered as important components of processing method when choosing processing strategy for certain type of FPCs. In this review, the processing strategies for FPCs are summarized into a "Toolbox" to cover recent progress as well as guide further research. The relationship of processing method-structure-property is discussed and the selection and combination of tools in processing from 700 studies among different FPCs are also analyzed.

In the "Toolbox" system, the mechanism in the field of FPCs is the cornerstones for the preparation of FPCs. Under its operation, we have a more directional choice of processing methods and meet the design requirements of FPCs better. The "Toolbox" consists processing type, primary processing tools and secondary processing tools for better understanding of the processing methods. The processing methods in the "Toolbox" are tools available to prepare FPCs, just like hammer or screwdriver in the engineering toolbox. The establishment of such "Toolbox" not only categorizes and summarizes the progress of current processing methods, but also is expected to guide the design of FPCs in the future. Based on the information given by the "Toolbox," the processing methods from different fields can learn from each other. Preparation methods commonly used in a specific 


\section{Structure-oriented research objectives: conductivity with anisotropy (ACF)}

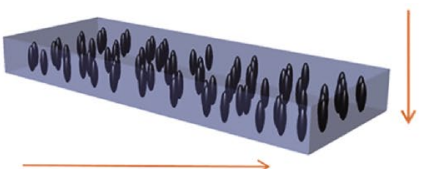

Horizontal nonconductivity
Vertical conductivity

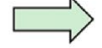

Required structure:

Filler orientation is

perpendicular to film orientation.

Toolbox combined with Al intelligence system Analyze and export optimization options for the design and processing of FPCs

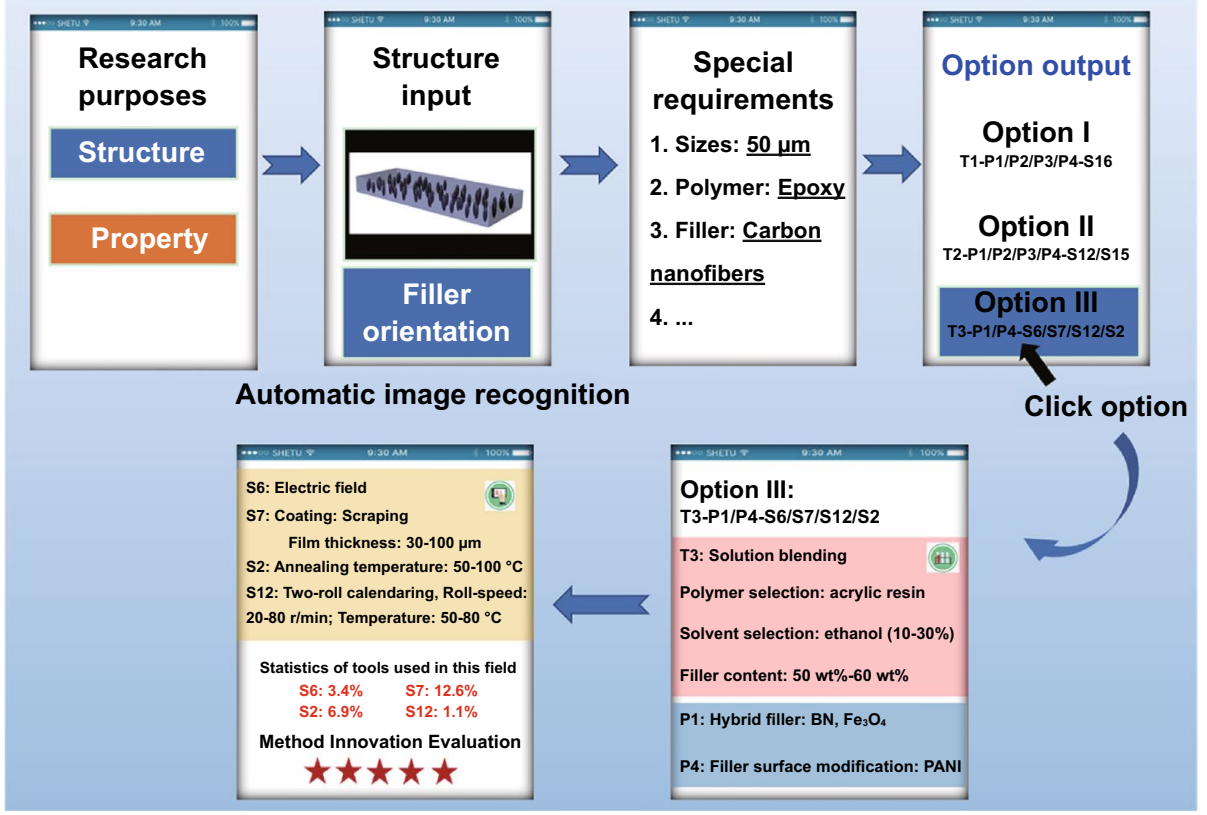

Fig. 15 Demonstration of the application for morphological control "Toolbox" in combination with artificial intelligence

field may bring fresh ideas for the preparation of FPCs in another field. Furthermore, new methods could be created by combining tools from "Toolbox" with different sequence as well as quantity. The combination of tools is not randomly done, it is understood that these tools are combined together with certain suitability due to various issues.

The future application trend of such "Toolbox" is prospected in this review. It is speculated that prediction on the processing tools applicable for certain FPCs could be made by computer software once "big data" on processing-structure-properties relationships of various FPCs is collected and summarized. By combining with "artificial intelligence" (AI) computer program, the concept of "Toolbox" program could be used to collect and summarize the overall materials database, and then, possible processing routes for given structure or targeted properties could be predicted and designed. It is thought that various pieces of information on certain study should be collected for the database: (i) the filler network structure realized by various processing method; (ii) the degree of improvement in certain functionality due to the utilization of different processing method; (iii) the type of polymers and fillers used for respective processing methods; (iv) the processing condition often used for certain type of processing methods. As shown in Fig. 15, structure purpose is used as an example to demonstrate the application for morphological control "Toolbox" in combination with artificial intelligence.

The design of materials with anisotropic conductivity is used as an example, where vertical conductivity is preferred. Firstly, we click to enter the intelligent front-end interface, select the design FPCs for the structure purpose, next upload the structure of our target, and then enter the next interface to put forward some special requirements such as size, filler and polymer matrix. After calculation, the optimal solutions of option-I, option-II and option-III would be exported for 
our choice. All this is based on the comprehensive database of the Toolbox. Besides, based on the system of database collection, "Toolbox" could also be used for performance evaluation. In short, the establishment of the "Toolbox" database has three benefits: (i) It is the cooperation of an open sharing platform; (ii) it is to efficiently implement the research and development of new functional materials by means of self-learning ability of artificial intelligence; and (iii) it can greatly shorten the research and development cycle of FPCs. Therefore, such method could be used to guide the future application of FPCs to achieve outstanding performance more efficiently and effectively.

Acknowledgements We gratefully acknowledge the financial support of this work by National Natural Science Foundation of China (51922071, 51773139).

Funding Open access funding provided by Shanghai Jiao Tong University.

Open Access This article is licensed under a Creative Commons Attribution 4.0 International License, which permits use, sharing, adaptation, distribution and reproduction in any medium or format, as long as you give appropriate credit to the original author(s) and the source, provide a link to the Creative Commons licence, and indicate if changes were made. The images or other third party material in this article are included in the article's Creative Commons licence, unless indicated otherwise in a credit line to the material. If material is not included in the article's Creative Commons licence and your intended use is not permitted by statutory regulation or exceeds the permitted use, you will need to obtain permission directly from the copyright holder. To view a copy of this licence, visit http://creativecommons.org/licenses/by/4.0/.

Supplementary Information The online version contains supplementary material available at https://doi.org/10.1007/ s40820-021-00774-5.

\section{References}

1. H. Deng, T. Skipa, E. Bilotti, R. Zhang, D. Lellinger et al., Preparation of high-performance conductive polymer fibers through morphological control of networks formed by nanofillers. Adv. Funct. Mater. 20(9), 1424-1432 (2010). https:// doi.org/10.1002/adfm.200902207

2. J. Li, B. Wang, Z. Ge, R. Cheng, L. Kang et al., Flexible and hierarchical 3D interconnected silver nanowires/cellulosic paper-based thermoelectric sheets with superior electrical conductivity and ultrahigh thermal dispersion capability. ACS Appl. Mater. Interfaces 11(42), 39088-39099 (2019). https://doi.org/10.1021/acsami.9b13675
3. T.Q. Trung, N.E. Lee, Recent progress on stretchable electronic devices with intrinsically stretchable components. Adv. Mater. 29(3), 1603167 (2017). https://doi.org/10.1002/adma. 201603167

4. X. Wang, X. Liu, D.W. Schubert, Highly sensitive ultrathin flexible thermoplastic polyurethane/carbon black fibrous film strain sensor with adjustable scaffold networks. Nano-Micro Lett. 13, 64 (2021). https://doi.org/10.1007/ s40820-021-00592-9

5. S.A. Moshizi, S. Azadi, A. Belford, A. Razmjou, S. Wu et al., Development of an ultra-sensitive and flexible piezoresistive flow sensor using vertical graphene nanosheets. Nano-Micro Lett. 12, 109 (2020). https://doi.org/10.1007/ s40820-020-00446-w

6. J. Chen, X. Huang, B. Sun, P. Jiang, Highly thermally conductive yet electrically insulating polymer/boron nitride nanosheets nanocomposite films for improved thermal management capability. ACS Nano 13(1), 337-345 (2019). https://doi.org/10.1021/acsnano.8b06290

7. F. Xiao, S. Naficy, G. Casillas, M.H. Khan, T. Katkus et al., Edge-hydroxylated boron nitride nanosheets as an effective additive to improve the thermal response of hydrogels. Adv. Mater. 27(44), 7196-7203 (2015). https://doi.org/10.1002/ adma.201502803

8. X. Yang, S. Fan, Y. Li, Y. Guo, Y. Li et al., Synchronously improved electromagnetic interference shielding and thermal conductivity for epoxy nanocomposites by constructing 3D copper nanowires/thermally annealed graphene aerogel framework. Compos. Part A Appl. Sci. Manuf. 128, 105670 (2020). https://doi.org/10.1016/j.compositesa.2019.105670

9. T. Juntunen, H. Jussila, M. Ruoho, S. Liu, G. Hu et al., Inkjet printed large-area flexible few-layer graphene thermoelectrics. Adv. Funct. Mater. 28(22), 1800480 (2018). https://doi. org/10.1002/adfm.201800480

10. Y. Wang, J. Yang, L. Wang, K. Du, Q. Yin et al., Polypyrrole/ graphene/polyaniline ternary nanocomposite with high thermoelectric power factor. ACS Appl. Mater. Interfaces 9(23), 20124-20131 (2017). https://doi.org/10.1021/acsami.7b053 57

11. Z. Wang, N.M. Han, Y. Wu, X. Liu, X. Shen et al., Ultrahigh dielectric constant and low loss of highly-aligned graphene aerogel/poly(vinyl alcohol) composites with insulating barriers. Carbon 123, 385-394 (2017). https://doi.org/10.1016/j. carbon.2017.07.079

12. K. Zhang, Z. Ma, H. Deng, Q. Fu, Improving high-temperature energy storage performance of PI dielectric capacitor films through boron nitride interlayer. Adv. Compos. Hybrid Mater. (2021). https://doi.org/10.1007/s42114-021-00329-7

13. X.X. Jin, J.F. Wang, L.Z. Dai, X.Y. Liu, L. Li et al., Flameretardant poly(vinyl alcohol)/MXene multilayered films with outstanding electromagnetic interference shielding and thermal conductive performances. Chem. Eng. J. 380, 122475 (2020). https://doi.org/10.1016/j.cej.2019.122475

14. C. Liang, Z. Gu, Y. Zhang, Z. Ma, H. Qiu et al., Structural design strategies of polymer matrix composites for 
electromagnetic interference shielding: a review. Nano Micro Lett. 13, 181 (2021). https://doi.org/10.1007/ s40820-021-00707-2

15. G. Zhao, X. Cao, Q. Zhang, H. Deng, Q. Fu, A novel interpenetrating segregated functional filler network structure for ultra-high electrical conductivity and efficient EMI shielding in CPCs containing carbon nanotubes. Mater. Today Phys. 21, 100438 (2021). https://doi.org/10.1016/j.mtphys.2021. 100483

16. H. Cai, B. Cheng, H. Xiao, Q. Wei, Synthesis of rGO/p$\mathrm{Fe}_{3} \mathrm{O}_{4} @$ PANi three-phase nanomaterials and electromagnetic wave absorption properties. Mater. Res. Express 6(12), 125621 (2019). https://doi.org/10.1088/2053-1591/ab5727

17. J.H. Pu, X.J. Zha, M. Zhao, S. Li, R.Y. Bao et al., 2D end-toend carbon nanotube conductive networks in polymer nanocomposites: a conceptual design to dramatically enhance the sensitivities of strain sensors. Nanoscale 10(5), 2191-2198 (2018). https://doi.org/10.1039/c7nr08077h

18. T. Kuang, L. Chang, F. Chen, Y. Sheng, D. Fu et al., Facile preparation of lightweight high-strength biodegradable polymer/multi-walled carbon nanotubes nanocomposite foams for electromagnetic interference shielding. Carbon 105, 305-313 (2016). https://doi.org/10.1016/j.carbon.2016.04.052

19. S. Luo, Y. Shen, S. Yu, Y. Wan, W.H. Liao et al., Construction of a $3 \mathrm{D}-\mathrm{BaTiO}_{3}$ network leading to significantly enhanced dielectric permittivity and energy storage density of polymer composites. Energy Environ. Sci. 10(1), 137-144 (2017). https://doi.org/10.1039/c6ee03190k

20. D.X. Yan, H. Pang, B. Li, R. Vajtai, L. Xu et al., Structured reduced graphene oxide/polymer composites for ultra-efficient electromagnetic interference shielding. Adv. Funct. Mater. 25(4), 559-566 (2015). https://doi.org/10.1002/adfm. 201403809

21. Y. Guo, G. Xu, X. Yang, K. Ruan, T. Ma et al., Significantly enhanced and precisely modeled thermal conductivity in polyimide nanocomposites with chemically modified graphene via in situ polymerization and electrospinning-hot press technology. J. Mater. Chem. C 6(12), 3004-3015 (2018). https:// doi.org/10.1039/c8tc00452h

22. V. Guerra, C.Y. Wan, T. McNally, Thermal conductivity of 2D nano-structured boron nitride (BN) and its composites with polymers. Prog. Mater. Sci. 100, 170-186 (2019). https://doi.org/10.1016/j.pmatsci.2018.10.002

23. H. Deng, L. Lin, M.Z. Ji, S.M. Zhang, M.B. Yang et al., Progress on the morphological control of conductive network in conductive polymer composites and the use as electroactive multifunctional materials. Prog. Polym. Sci. 39(4), 627-655 (2014). https://doi.org/10.1016/j.progpolymsci.2013.07.007

24. H. Abbasi, M. Antunes, J.I. Velasco, Recent advances in carbon-based polymer nanocomposites for electromagnetic interference shielding. Prog. Mater. Sci. 103, 319-373 (2019). https://doi.org/10.1016/j.pmatsci.2019.02.003

25. H. Deng, Q. Fu, Recent progress on the confinement, assembly, and relaxation of inorganic functional fillers in polymer matrix during processing. Macromol. Rapid Commun.
38(23), 1700444 (2017). https://doi.org/10.1002/marc.20170 0444

26. H. Liu, Q. Li, S. Zhang, R. Yin, X. Liu et al., Electrically conductive polymer composites for smart flexible strain sensors: a critical review. J. Mater. Chem. C 6(45), 12121-12141 (2018). https://doi.org/10.1039/c8tc04079f

27. X. Huang, P. Jiang, T. Tanaka, A review of dielectric polymer composites with high thermal conductivity. IEEE Electr. Insul. Mag. 27(4), 8-16 (2011). https://doi.org/10.1109/mei. 2011.5954064

28. N. Burger, A. Laachachi, M. Ferriol, M. Lutz, V. Toniazzo et al., Review of thermal conductivity in composites: mechanisms, parameters and theory. Prog. Polym. Sci. 61, 1-28 (2016). https://doi.org/10.1016/j.progpolymsci.2016.05.001

29. T.D. Huan, S. Boggs, G. Teyssedre, C. Laurent, M. Cakmak et al., Advanced polymeric dielectrics for high energy density applications. Prog. Mater. Sci. 83, 236-269 (2016). https:// doi.org/10.1016/j.pmatsci.2016.05.001

30. G. Chen, W. Xu, D. Zhu, Recent advances in organic polymer thermoelectric composites. J. Mater. Chem. C 5(18), 4350-4360 (2017). https://doi.org/10.1039/c6tc05488a

31. H. Alam, S. Ramakrishna, A review on the enhancement of figure of merit from bulk to nano-thermoelectric materials. Nano Energy 2(2), 190-212 (2013). https://doi.org/10.1016/j. nanoen.2012.10.005

32. P. Kumar, U.N. Maiti, A. Sikdar, T.K. Das, A. Kumar et al., Recent advances in polymer and polymer composites for electromagnetic interference shielding: review and future prospects. Polym. Rev. 59(4), 687-738 (2019). https://doi. org/10.1080/15583724.2019.1625058

33. K. Ruan, X. Zhong, X. Shi, J. Dang, J. Gu, Liquid crystal epoxy resins with high intrinsic thermal conductivities and their composites: a mini-review. Mater. Today Phys. 20, 100456 (2021). https://doi.org/10.1016/j.mtphys.2021.100456

34. L. Zhang, H. Deng, Q. Fu, Recent progress on thermal conductive and electrical insulating polymer composites. Compos. Commun. 8, 74-82 (2018). https://doi.org/10.1016/j. coco.2017.11.004

35. K. Ke, Y. Wang, K. Zhang, Y. Luo, W. Yang et al., Melt viscoelasticity, electrical conductivity, and crystallization of PVDF/MWCNT composites: effect of the dispersion of MWCNTs. J. Appl. Polym. Sci. 125, 49-57 (2012). https:// doi.org/10.1002/app.36293

36. G. Chen, Y. Ma, Z. Qi, Preparation and morphological study of an exfoliated polystyrene/montmorillonite nanocomposite. Scr. Mater. 44(1), 125-128 (2001). https://doi.org/10.1016/ S1359-6462(00)00563-7

37. K. Xu, G. Chen, D. Qiu, Convenient construction of poly(3,4ethylenedioxythiophene)-graphene pie-like structure with enhanced thermoelectric performance. J. Mater. Chem. A 1(40), 12395-12399 (2013). https://doi.org/10.1039/c3ta1 2691a

38. X. Hu, G. Chen, X. Wang, An unusual coral-like morphology for composites of poly(3,4-ethylenedioxythiophene)/carbon nanotube and the enhanced thermoelectric performance. 
Compos. Sci. Technol. 144, 43-50 (2017). https://doi.org/ 10.1016/j.compscitech.2017.03.018

39. Z. Zhang, G. Chen, H. Wang, W. Zhai, Enhanced thermoelectric property by the construction of a nanocomposite 3D interconnected architecture consisting of graphene nanolayers sandwiched by polypyrrole nanowires. J. Mater. Chem. C 3(8), 1649-1654 (2015). https://doi.org/10.1039/c4tc02471k

40. M. Terrones, O. Martin, M. Gonzalez, J. Pozuelo, B. Serrano et al., Interphases in graphene polymer-based nanocomposites: achievements and challenges. Adv. Mater. 23(44), 5302-5310 (2011). https://doi.org/10.1002/adma.201102036

41. W. Deng, L. Deng, Z. Li, Y. Zhang, G. Chen, Synergistically boosting thermoelectric performance of PEDOT: PSS/ SWCNT composites via the ion-exchange effect and promoting SWCNT dispersion by the ionic liquid. ACS Appl. Mater. Interfaces 13(10), 12131-12140 (2021). https://doi.org/10. 1021/acsami.1c01059

42. L. Liang, G. Chen, C. Guo, Enhanced thermoelectric performance by self-assembled layered morphology of polypyrrole nanowire/single-walled carbon nanotube composites. Compos. Sci. Technol. 129, 130-136 (2016). https://doi.org/10. 1016/j.compscitech.2016.04.023

43. M. Liao, P. Wan, J. Wen, M. Gong, X. Wu et al., Wearable, healable, and adhesive epidermal sensors assembled from mussel-inspired conductive hybrid hydrogel framework. Adv. Funct. Mater. 27(48), 1703852 (2017). https://doi.org/ 10.1002/adfm.201703852

44. K. Takei, Z. Yu, M. Zheng, H. Ota, T. Takahashi et al., Highly sensitive electronic whiskers based on patterned carbon nanotube and silver nanoparticle composite films. PNAS 111(5), 1703-1707 (2014). https://doi.org/10.1073/pnas.1317920111

45. S. Wang, X. Zhang, X. Wu, C. Lu, Tailoring percolating conductive networks of natural rubber composites for flexible strain sensors via a cellulose nanocrystal templated assembly. Soft Matter 12(3), 845-852 (2016). https://doi.org/10.1039/ c5sm01958c

46. M. Wang, K. Zhang, X.X. Dai, Y. Li, J. Guo et al., Enhanced electrical conductivity and piezoresistive sensing in multiwall carbon nanotubes/polydimethylsiloxane nanocomposites via the construction of a self-segregated structure. Nanoscale 9(31), 11017-11026 (2017). https://doi.org/10.1039/c7nr0 $2322 \mathrm{~g}$

47. S. Liu, V.S. Chevali, Z. Xu, D. Hui, H. Wang, A review of extending performance of epoxy resins using carbon nanomaterials. Compos. Part B Eng. 136, 197-214 (2018). https:// doi.org/10.1016/j.compositesb.2017.08.020

48. Z. Ahmadi, Nanostructured epoxy adhesives: a review. Prog. Org. Coat. 135, 449-453 (2019). https://doi.org/10.1016/j. porgcoat.2019.06.028

49. Y. Yang, Z. Li, W. Ji, C. Sun, H. Deng et al., Enhanced dielectric properties through using mixed fillers consisting of nanobarium titanate/nickel hydroxide for polyvinylidene fluoride based composites. Compos. Part A Appl. Sci. Manuf. 104, 24-31 (2018). https://doi.org/10.1016/j.compositesa.2017.10. 024
50. H. Im, J. Kim, Thermal conductivity of a graphene oxidecarbon nanotube hybrid/epoxy composite. Carbon 50(15), 5429-5440 (2012). https://doi.org/10.1016/j.carbon.2012. 07.029

51. D. Xiang, L. Wang, Y. Tang, C. Zhao, E. Harkinjones et al., Effect of phase transitions on the electrical properties of polymer/carbon nanotube and polymer/graphene nanoplatelet composites with different conductive network structures. Polym. Int. 67(2), 227-235 (2018). https://doi.org/10.1002/ pi.5502

52. G. Yang, J. Tang, Q. Fu, A facile melt coating approach to fabricate macroscopic segregated polymer/carbon nanotube conductive composites with balanced properties. Polym. Compos. 39(3), 841-847 (2018). https://doi.org/10.1002/pc. 24006

53. O. Maruzhenko, Y. Mamunya, G. Boiteux, S. Pusz, U. Szeluga et al., Improving the thermal and electrical properties of polymer composites by ordered distribution of carbon microand nanofillers. Int. J. Heat Mass Transf. 138, 75-84 (2019). https://doi.org/10.1016/j.ijheatmasstransfer.2019.04.043

54. S. Yang, W. Li, S. Bai, Q. Wang, High-performance thermal and electrical conductive composites from multilayer plastic packaging waste and expanded graphite. J. Mater. Chem. C 6(41), 11209-11218 (2018). https://doi.org/10.1039/c8tc0 2840k

55. S. Wang, Y. Zhou, Y. Liu, L. Wang, C. Gao, Enhanced thermoelectric properties of polyaniline/polypyrrole/carbon nanotube ternary composites by treatment with a secondary dopant using ferric chloride. J. Mater. Chem. C 8(2), 528-535 (2020). https://doi.org/10.1039/c9tc06300e

56. L. Duan, D.R. Dhooge, M. Spoerk, P. Cornillie, L. Cardon, Facile and low-cost route for sensitive stretchable sensors by controlling kinetic and thermodynamic conductive network regulating strategies. ACS Appl. Mater. Interfaces 10(26), 22678-22691 (2018). https://doi.org/10.1021/acsami.8b039 67

57. R. Dou, Y. Shao, S. Li, B. Yin, M. Yang, Structuring tri-continuous structure multiphase composites with ultralow conductive percolation threshold and excellent electromagnetic shielding effectiveness using simple melt mixing. Polymer 83, 34-39 (2016). https://doi.org/10.1016/j.polymer.2015.12. 005

58. L. Li, S. Li, Y. Shao, R. Dou, B. Yin et al., PVDF/PS/HDPE/ MWCNTs/ $/ \mathrm{Fe}_{3} \mathrm{O}_{4}$ nanocomposites: effective and lightweight electromagnetic interference shielding material through the synergetic effect of MWCNTs and $\mathrm{Fe}_{3} \mathrm{O}_{4}$ nanoparticles. Curr. Appl. Phys. 18(4), 388-396 (2018). https://doi.org/10.1016/j. cap.2018.01.014

59. S.A. Paniagua, Y. Kim, K. Henry, R. Kumar, J.W. Perry et al., Surface-initiated polymerization from barium titanate nanoparticles for hybrid dielectric capacitors. ACS Appl. Mater. Interfaces 6(5), 3477-3482 (2014). https://doi.org/10.1021/ am4056276

60. Z. Weng, R. Guan, F. Zou, P. Zhou, Y. Liao et al., A highly sensitive polydopamine@ hybrid carbon nanofillers based nanocomposite sensor for acquiring high-frequency 
ultrasonic waves. Carbon 170, 403-413 (2020). https://doi. org/10.1016/j.carbon.2020.08.030

61. X. Zhang, C. Tan, Y. Ma, F. Wang, W. Yang, BaTiO 3 carbon/silicon carbide/poly(vinylidene fluoride-hexafluoropropylene) three-component nanocomposites with high dielectric constant and high thermal conductivity. Compos. Sci. Technol. 162, 180-187 (2018). https://doi.org/10.1016/j. compscitech.2018.05.001

62. X. Huang, P. Jiang, Core-shell structured high- $k$ polymer nanocomposites for energy storage and dielectric applications. Adv. Mater. 27(3), 546-554 (2015). https://doi.org/10. 1002/adma.201401310

63. C.J. Galvin, J. Genzer, Applications of surface-grafted macromolecules derived from post-polymerization modification reactions. Prog. Polym. Sci. 37(7), 871-906 (2012). https:// doi.org/10.1016/j.progpolymsci.2011.12.001

64. M.P. Wolf, G.B. Salieb-Beugelaar, P. Hunziker, PDMS with designer functionalities-properties, modifications strategies, and applications. Prog. Polym. Sci. 83, 97-134 (2018). https://doi.org/10.1016/j.progpolymsci.2018.06.001

65. M. Antunes, J.I. Velasco, Multifunctional polymer foams with carbon nanoparticles. Prog. Polym. Sci. 39(3), 486-509 (2014). https://doi.org/10.1016/j.progpolymsci.2013.11.002

66. D. Raps, N. Hossieny, C.B. Park, V. Altstädt, Past and present developments in polymer bead foams and bead foaming technology. Polymer 56, 5-19 (2015). https://doi.org/10.1016/j. polymer.2014.10.078

67. G. Sang, P. Xu, T. Yan, V. Murugadoss, N. Naik et al., Interface engineered microcellular magnetic conductive polyurethane nanocomposite foams for electromagnetic interference shielding. Nano-Micro Lett. 13, 153 (2021). https://doi.org/ 10.1007/s40820-021-00677-5

68. N. Petchwattana, S. Covavisaruch, Influences of particle sizes and contents of chemical blowing agents on foaming wood plastic composites prepared from poly(vinyl chloride) and rice hull. Mater. Design. 32(5), 2844-2850 (2011). https:// doi.org/10.1016/j.matdes.2010.12.044

69. H. Zhang, G. Zhang, M. Tang, L. Zhou, J. Li et al., Synergistic effect of carbon nanotube and graphene nanoplates on the mechanical, electrical and electromagnetic interference shielding properties of polymer composites and polymer composite foams. Chem. Eng. J. 353, 381-393 (2018). https://doi.org/10.1016/j.cej.2018.07.144

70. J. Ma, M. Zhan, K. Wang, Ultralightweight silver nanowires hybrid polyimide composite foams for high-performance electromagnetic interference shielding. ACS Appl. Mater. Interfaces 7, 563-576 (2015). https://doi.org/10.1021/am5067095

71. J. Li, G. Zhang, Z. Ma, X. Fan, X. Fan et al., Morphologies and electromagnetic interference shielding performances of microcellular epoxy/multi-wall carbon nanotube nanocomposite foams. Compos. Sci. Technol. 129, 70-78 (2016). https://doi.org/10.1016/j.compscitech.2016.04.003

72. S. Xu, X. Li, G. Sui, R. Du, Q. Zhang et al., Plasma modification of PU foam for piezoresistive sensor with high sensitivity, mechanical properties and long-term stability. Chem.
Eng. J. 381, 122666 (2020). https://doi.org/10.1016/j.cej. 2019.122666

73. Y. Pang, H. Tian, L. Tao, Y. Li, X. Wang et al., Flexible, highly sensitive, and wearable pressure and strain sensors with graphene porous network structure. ACS Appl. Mater. Interfaces 8(40), 26458-26462 (2016). https://doi.org/10. 1021/acsami.6b08172

74. Y.C. Zhang, H. Pang, K. Dai, Y.F. Huang, P.G. Ren et al., Conductive network formation during annealing of an oriented polyethylene-based composite. J. Mater. Sci. 47(8), 3713-3719 (2011). https://doi.org/10.1007/ s10853-011-6220-2

75. Y. Xue, Z. Zhang, Y. Zhang, X. Wang, L. Li et al., Boosting thermoelectric performance by in situ growth of metal organic framework on carbon nanotube and subsequent annealing. Carbon 157, 324-329 (2020). https://doi.org/10. 1016/j.carbon.2019.10.049

76. W. Gao, Y. Zheng, J. Shen, S. Guo, Electrical properties of polypropylene-based composites controlled by multilayered distribution of conductive particles. ACS Appl. Mater. Interfaces 7(3), 1541-1549 (2015). https://doi.org/10.1021/am506773c

77. Z. Li, A. Olah, E. Baer, Micro- and nano-layered processing of new polymeric systems. Prog. Polym. Sci. 102, 101210 (2020). https://doi.org/10.1016/j.progpolymsci.2020.101210

78. X. Zhang, Y. Xu, X. Zhang, H. Wu, J. Shen et al., Progress on the layer-by-layer assembly of multilayered polymer composites: strategy, structural control and applications. Prog. Polym. Sci. 89, 76-107 (2019). https://doi.org/10.1016/j. progpolymsci.2018.10.002

79. F. Yu, H. Deng, Q. Zhang, K. Wang, C. Zhang et al., Anisotropic multilayer conductive networks in carbon nanotubes filled polyethylene/polypropylene blends obtained through high speed thin wall injection molding. Polymer 54(23), 6425-6436 (2013). https://doi.org/10.1016/j.polymer.2013. 09.047

80. F. Wang, L. Liu, P. Xue, M. Jia, S. Wang et al., The influence of formation temperatures on the crystal structure and mechanical properties of ultrahigh-molecular-weight polyethylene/high-density polyethylene-blend fibers prepared by melt spinning. J. Ind. Text. 49(8), 1011-1035 (2019). https:// doi.org/10.1177/1528083719827371

81. J.Y. Kim, J.H. Mo, Y.H. Kang, S.Y. Cho, K.S. Jang, Thermoelectric fibers from well-dispersed carbon nanotube/ poly(vinyliedene fluoride) pastes for fiber-based thermoelectric generators. Nanoscale 10(42), 19766-19773 (2018). https://doi.org/10.1039/c8nr06415f

82. Y. Huang, J. Song, C. Yang, Y. Long, H. Wu, Scalable manufacturing and applications of nanofibers. Mater. Today 28, 98-113 (2019). https://doi.org/10.1016/j.mattod.2019.04.018

83. R. Pantani, I. Coccorullo, V. Speranza, G. Titomanlio, Modeling of morphology evolution in the injection molding process of thermoplastic polymers. Prog. Polym. Sci. 30(12), 1185-1222 (2005). https://doi.org/10.1016/j.progpolymsci. 2005.09.001

84. M.R. Khosravani, S. Nasiri, Injection molding manufacturing process: review of case-based reasoning applications. 
J. Intell. Manuf. 31(4), 847-864 (2019). https://doi.org/10. 1007/s10845-019-01481-0

85. H. Yui, G. Wu, H. Sano, M. Sumita, K. Kino, Morphology and electrical conductivity of injection-molded polypropylene/carbon black composites with addition of high-density polyethylene. Polymer 47(10), 3599-3608 (2006). https://doi. org/10.1016/j.polymer.2006.03.064

86. M. Arjmand, T. Apperley, M. Okoniewski, U. Sundararaj, Comparative study of electromagnetic interference shielding properties of injection molded versus compression molded multi-walled carbon nanotube/polystyrene composites. Carbon 50(14), 5126-5134 (2012). https://doi.org/10.1016/j. carbon.2012.06.053

87. K. Maghsoudi, R. Jafari, G. Momen, M. Farzaneh, Micronanostructured polymer surfaces using injection molding: a review. Mater. Today Commun. 13, 126-143 (2017). https:// doi.org/10.1016/j.mtcomm.2017.09.013

88. S. Xu, D. Liu, Q. Zhang, Q. Fu, Electric field-induced alignment of nanofibrillated cellulose in thermoplastic polyurethane matrix. Compos. Sci. Technol. 156, 117-126 (2018). https://doi.org/10.1016/j.compscitech.2017.12.017

89. Y. Chen, M. He, J. Tang, G.C. Bazan, Z. Liang, Flexible thermoelectric generators with ultrahigh output power enabled by magnetic field-aligned metallic nanowires. Adv. Electron. Mater. 4(9), 1800200 (2018). https://doi.org/10.1002/aelm. 201800200

90. K. Kim, H. Ju, J. Kim, Vertical particle alignment of boron nitride and silicon carbide binary filler system for thermal conductivity enhancement. Compos. Sci. Technol. 123, 99-105 (2016). https://doi.org/10.1016/j.compscitech.2015. 12.004

91. C.T. Hong, Y.H. Kang, J. Ryu, S.Y. Cho, K.S. Jang, Sprayprinted CNT/P3HT organic thermoelectric films and power generators. J. Mater. Chem. A 3(43), 21428-21433 (2015). https://doi.org/10.1039/c5ta06096f

92. H. Shen, J. Guo, H. Wang, N. Zhao, J. Xu, Bioinspired modification of h-BN for high thermal conductive composite films with aligned structure. ACS Appl. Mater. Interfaces 7(10), 5701-5708 (2015). https://doi.org/10.1021/am507416y

93. X. Liang, T. Zhao, P. Zhu, Y. Hu, R. Sun et al., Room-temperature nanowelding of a silver nanowire network triggered by hydrogen chloride vapor for flexible transparent conductive films. ACS Appl. Mater. Interfaces 9(46), 40857-40867 (2017). https://doi.org/10.1021/acsami.7b13048

94. M. Kim, T.W. Lee, S.M. Park, Y.G. Jeong, Structures, electrical and mechanical properties of epoxy composites reinforced with MWCNT-coated basalt fibers. Compos. Part A Appl. Sci. Manuf. 123, 123-131 (2019). https://doi.org/10.1016/j. compositesa.2019.05.011

95. Q. Zheng, Z. Li, J. Yang, J.K. Kim, Graphene oxide-based transparent conductive films. Prog. Mater. Sci. 64, 200-247 (2014). https://doi.org/10.1016/j.pmatsci.2014.03.004

96. H. Xu, M. Qu, D.W. Schubert, Conductivity of poly(methyl methacrylate) composite films filled with ultra-high aspect ratio carbon fibers. Compos. Sci. Technol. 181, 107690 (2019). https://doi.org/10.1016/j.compscitech.2019.107690
97. S. Wang, P. Xiao, Y. Liang, J. Zhang, Y. Huang et al., Network cracks-based wearable strain sensors for subtle and large strain detection of human motions. J. Mater. Chem. C 6(19), 5140-5147 (2018). https://doi.org/10.1039/c8tc00433a

98. Z. Niu, H. Dong, B. Zhu, J. Li, H.H. Hng et al., Highly stretchable, integrated supercapacitors based on single-walled carbon nanotube films with continuous reticulate architecture. Adv. Mater. 25(7), 1058-1064 (2013). https://doi.org/ 10.1002/adma.201204003

99. J. Ge, L. Sun, F.R. Zhang, Y. Zhang, L.A. Shi et al., A stretchable electronic fabric artificial skin with pressure, lateral strain and flexion-sensitive properties. Adv. Mater. 28(4), 722-728 (2016). https://doi.org/10.1002/adma.201504239

100. M. Zu, Q. Li, G. Wang, J.H. Byun, T.W. Chou, Carbon nanotube fiber based stretchable conductor. Adv. Funct. Mater. 23(7), 789-793 (2013). https://doi.org/10.1002/adfm.201202174

101. Y. Yu, S. Luo, L. Sun, Y. Wu, K. Jiang et al., Ultra-stretchable conductors based on buckled super-aligned carbon nanotube films. Nanoscale 7(22), 10178-10185 (2015). https://doi.org/ $10.1039 / \mathrm{c} 5 \mathrm{nr} 01383 \mathrm{f}$

102. A. Marconnet, N. Yamamoto, M.A. Panzer, B.L. Wardle, K.E. Goodson, Thermal conduction in aligned carbon nanotube polymer nanocomposites with high packing density. ACS Nano 5(6), 4818-4852 (2011). https://doi.org/10.1021/nn200 $847 \mathrm{u}$

103. Y. Chen, H.B. Zhang, Y. Yang, M. Wang, A. Cao et al., Highperformance epoxy nanocomposites reinforced with threedimensional carbon nanotube sponge for electromagnetic interference shielding. Adv. Funct. Mater. 26(3), 447-455 (2016). https://doi.org/10.1002/adfm.201503782

104. Y. Huangfu, C. Liang, Y. Han, H. Qiu, P. Song et al., Fabrication and investigation on the $\mathrm{Fe}_{3} \mathrm{O}_{4}$ /thermally annealed graphene aerogel/epoxy electromagnetic interference shielding nanocomposites. Compos. Sci. Technol. 169, 70-75 (2019). https://doi.org/10.1016/j.compscitech.2018.11.012

105. J.K.Y. Lee, N. Chen, S. Peng, L. Li, L. Tian et al., Polymerbased composites by electrospinning: preparation \& functionalization with nanocarbons. Prog. Polym. Sci. 86, 40-84 (2018). https://doi.org/10.1016/j.progpolymsci.2018.07.002

106. H. Chen, N. Wang, J. Di, Y. Zhao, Y. Song et al., Nanowirein-microtube structured core/shell fibers via multifluidic coaxial electrospinning. Langmuir 26(13), 11291-11296 (2010). https://doi.org/10.1021/la100611f

107. Y. Jin, D. Yang, D. Kang, X. Jiang, Fabrication of necklacelike structures via electrospinning. Langmuir 26(2), 11861190 (2010). https://doi.org/10.1021/la902313t

108. P. Lu, Y. Xia, Maneuvering the internal porosity and surface morphology of electrospun polystyrene yarns by controlling the solvent and relative humidity. Langmuir 29(23), 70707078 (2013). https://doi.org/10.1021/la400747y

109. Z. He, Q. Liu, H. Hou, F. Gao, B. Tang et al., Tailored electrospinning of $\mathrm{WO}_{3}$ nanobelts as efficient ultraviolet photodetectors with photo-dark current ratios up to 1000. ACS Appl. Mater. Interfaces 7(20), 10878-10885 (2015). https://doi.org/ 10.1021/acsami.5b02020 
110. Y. Gao, F. Guo, P. Cao, J. Liu, D. Li et al., Winding-locked carbon nanotubes/polymer nanofibers helical yarn for ultrastretchable conductor and strain sensor. ACS Nano 14(3), 3442-3450 (2020). https://doi.org/10.1021/acsnano.9b09533

111. Y. Guo, Z. Lyu, X. Yang, Y. Lu, K. Ruan et al., Enhanced thermal conductivities and decreased thermal resistances of functionalized boron nitride/polyimide composites. Compos. Part B Eng. 164, 732-739 (2019). https://doi.org/10.1016/j. compositesb.2019.01.099

112. I.W. Nam, H.K. Lee, J.H. Jang, Electromagnetic interference shielding/absorbing characteristics of CNT-embedded epoxy composites. Compos. Part A Appl. Sci. Manuf. 42(9), 1110 1118 (2011). https://doi.org/10.1016/j.compositesa.2011.04.016

113. R. Ravindren, S. Mondal, K. Nath, N.C. Das, Investigation of electrical conductivity and electromagnetic interference shielding effectiveness of preferentially distributed conductive filler in highly flexible polymer blends nanocomposites. Compos. Part A Appl. Sci. Manuf. 118, 75-89 (2019). https:// doi.org/10.1016/j.compositesa.2018.12.012

114. S. Deville, E. Saiz, R.K. Nalla, A.P. Tomsia, Freezing as a path to build complex composites. Science 311(5760), 515518 (2006). https://doi.org/10.1126/science.1120937

115. J. Yang, E. Zhang, X. Li, Y. Zhang, J. Qu et al., Cellulose/ graphene aerogel supported phase change composites with high thermal conductivity and good shape stability for thermal energy storage. Carbon 98, 50-57 (2016). https://doi.org/ 10.1016/j.carbon.2015.10.082

116. Z. Zeng, H. Jin, M. Chen, W. Li, L. Zhou et al., Lightweight and anisotropic porous MWCNT/WPU composites for ultrahigh performance electromagnetic interference shielding. Adv. Funct. Mater. 26(2), 303-310 (2016). https://doi.org/ 10.1002/adfm.201503579

117. C. Wang, Z.Z. Pan, W. Lv, B. Liu, J. Wei et al., A directional strain sensor based on anisotropic microhoneycomb cellulose nanofiber-carbon nanotube hybrid aerogels prepared by unidirectional freeze drying. Small 15(14), 1805363 (2019). https://doi.org/10.1002/smll.201805363

118. J. Han, G. Du, W. Gao, H. Bai, An anisotropically high thermal conductive boron nitride/epoxy composite based on nacre-mimetic 3D network. Adv. Funct. Mater. 29(13), 1900412 (2019). https://doi.org/10.1002/adfm.201900412

119. R.K. Iler, Multilayers of colloidal particles. J. Colloid Interface Sci. 21(6), 569-594 (1966). https://doi.org/10.1016/ 0095-8522(66)90018-3

120. X. Wang, P. Wu, Melamine foam-supported 3D interconnected boron nitride nanosheets network encapsulated in epoxy to achieve significant thermal conductivity enhancement at an ultralow filler loading. Chem. Eng. J. 348, 723731 (2018). https://doi.org/10.1016/j.cej.2018.04.196

121. J. Heo, D. Choi, J. Hong, Layer-by-layer self-assembled ferrite multilayer nanofilms for microwave absorption. J. Nanomater. 2015, 1-8 (2015). https://doi.org/10.1155/2015/ 164619

122. M. Culebras, C. Cho, M. Krecker, R. Smith, Y. Song et al., High thermoelectric power factor organic thin films through combination of nanotube multilayer assembly and electrochemical polymerization. ACS Appl. Mater. Interfaces 9(7), 6306-6313 (2017). https://doi.org/10.1021/acsami. $6 \mathrm{~b} 15327$

123. Y. Guo, X. Yang, K. Ruan, J. Kong, M. Dong et al., Reduced graphene oxide heterostructured silver nanoparticles significantly enhanced thermal conductivities in hot-pressed electrospun polyimide nanocomposites. ACS Appl. Mater. Interfaces 11(28), 25465-25473 (2019). https://doi.org/10. 1021/acsami.9b10161

124. T. Wang, W.W. Kong, W.C. Yu, J.F. Gao, K. Dai et al., A healable and mechanically enhanced composite with segregated conductive network structure for high-efficient electromagnetic interference shielding. Nano-Micro Lett. 13, 162 (2021). https://doi.org/10.1007/s40820-021-00693-5

125. X. Yang, Y. Guo, Y. Han, Y. Li, T. Ma et al., Significant improvement of thermal conductivities for BNNs/PVA composite films via electrospinning followed by hot-pressing technology. Compos. Part B Eng. 175, 107070 (2019). https:// doi.org/10.1016/j.compositesb.2019.107070

126. G. Chen, S. Liu, S. Zhang, Z. Qi, Self-assembly in a polystyrene/montmorillonite nanocomposite. Macromol. Rapid Commun. 21(11), 746-749 (2000). https://doi.org/10.1002/ 1521-3927(20000701)21:11\%3c746::AID-MARC746\% 3e3.0.CO;2-K

127. G. Chen, D. Shen, M. Feng, M. Yang, An attenuated total reflection FT-IR spectroscopic study of polyamide 6/clay nanocomposite fibers. Macromol. Rapid Commun. 25, 11211124 (2004). https://doi.org/10.1002/marc.200400079

128. C. Yuan, J. Wang, G. Chen, J. Zhang, J. Yang, Orientation studies of uniaxial drawn syndiotactic polystyrene/carbon nanotube nanocomposite films. Soft Matter 7, 4039-4044 (2011). https://doi.org/10.1039/c0sm01475c

129. Y. Zhang, L. Deng, H. Lv, G. Chen, Toward improved tradeoff between thermoelectric and mechanical performances in polycarbonate/single-walled carbon nanotube composite films. npj Flex. Electron. 4, 26 (2020). https://doi.org/10. 1038/s41528-020-00089-2

130. M. Falahati, P. Ahmadvand, S. Safaee, Y.C. Chang, Z. Lyu et al., Smart polymers and nanocomposites for 3D and 4D printing. Mater. Today 40, 215-245 (2020). https://doi.org/ 10.1016/j.mattod.2020.06.001

131. X. Wang, M. Jiang, Z. Zhou, J. Gou, D. Hui, 3D printing of polymer matrix composites: a review and prospective. Compos. Part B Eng. 110, 442-458 (2017). https://doi.org/ 10.1016/j.compositesb.2016.11.034

132. A. Mostafaei, A.M. Elliott, J.E. Barnes, F. Li, W. Tan et al., Binder jet 3D printing - process parameters, materials, properties, and challenges. Prog. Mater. Sci. 119, 100707 (2021). https://doi.org/10.1016/j.pmatsci.2020.100684

133. A.L. Pires, I.F. Cruz, J. Silva, G.N.P. Oliveira, S. FerreiraTeixeira et al., Printed flexible $\mu$-thermoelectric device based on hybrid $\mathrm{Bi}_{2} \mathrm{Te}_{3} / \mathrm{PVA}$ composites. ACS Appl. Mater. Interfaces 11(9), 8969-8981 (2019). https://doi.org/10.1021/ acsami.8b18081

134. M. Vural, A. Pena-Francesch, J. Bars-Pomes, H. Jung, H. Gudapati et al., Inkjet printing of self-assembled 2D titanium 
carbide and protein electrodes for stimuli-responsive electromagnetic shielding. Adv. Funct. Mater. 28(32), 1801972 (2018). https://doi.org/10.1002/adfm.201801972

135. C.J. Zhang, L. McKeon, M.P. Kremer, S.H. Park, O. Ronan et al., Additive-free MXene inks and direct printing of microsupercapacitors. Nat. Commun. 10, 1795 (2019). https://doi. org/10.1038/s41467-019-09398-1

136. B.F. Gonçalves, J. Oliveira, P. Costa, V. Correia, P. Martins et al., Development of water-based printable piezoresistive sensors for large strain applications. Compos. Part B Eng. 112, 344-352 (2017). https://doi.org/10.1016/j.compositesb.2016.12.047

137. D. Zhang, B. Chi, B. Li, Z. Gao, Y. Du et al., Fabrication of highly conductive graphene flexible circuits by $3 \mathrm{D}$ printing. Synth. Met. 217, 79-86 (2016). https://doi.org/10.1016/j. synthmet.2016.03.014

138. T.D. Ngo, A. Kashani, G. Imbalzano, K.T.Q. Nguyen, D. Hui, Additive manufacturing (3D printing): a review of materials, methods, applications and challenges. Compos. Part B Eng. 143, 172-196 (2018). https://doi.org/10.1016/j.compositesb. 2018.02.012

139. X. Yang, Y. Guo, X. Luo, N. Zheng, T. Ma et al., Self-healing, recoverable epoxy elastomers and their composites with desirable thermal conductivities by incorporating BN fillers via in-situ polymerization. Compos. Sci. Technol. 164, 59-64 (2018). https://doi.org/10.1016/j.compscitech.2018.05.038

140. Z.A. Ghaleb, M. Mariatti, Z.M. Ariff, Synergy effects of graphene and multiwalled carbon nanotubes hybrid system on properties of epoxy nanocomposites. J. Reinf. Plast. Comp. 36(9), 685-695 (2017). https://doi.org/10.1177/0731684417 692055

141. Y. Li, B. Shen, D. Yi, L. Zhang, W. Zhai et al., The influence of gradient and sandwich configurations on the electromagnetic interference shielding performance of multilayered thermoplastic polyurethane/graphene composite foams. Compos. Sci. Technol. 138, 209-216 (2017). https://doi.org/10.1016/j. compscitech.2016.12.002

142. C. Wu, X. Huang, X. Wu, L. Xie, K. Yang et al., Graphene oxide-encapsulated carbon nanotube hybrids for high dielectric performance nanocomposites with enhanced energy storage density. Nanoscale 5(9), 3847-3855 (2013). https://doi. org $/ 10.1039 / \mathrm{c} 3 \mathrm{nr} 00625 \mathrm{e}$

143. Y. Zhang, Y. Wang, Y. Deng, M. Li, J. Bai, Enhanced dielectric properties of ferroelectric polymer composites induced by metal-semiconductor $\mathrm{Zn}-\mathrm{ZnO}$ core-shell structure. ACS Appl. Mater. Interfaces 4, 65-68 (2012). https://doi.org/10. 1021/am2016156

144. C. Ou, A.L. Sangle, A. Datta, Q. Jing, T. Busolo et al., Fully printed organic-inorganic nanocomposites for flexible thermoelectric applications. ACS Appl. Mater. Interfaces 10(23), 19580-19587 (2018). https://doi.org/10.1021/acsami.8b014 56

145. R. Zhang, A. Lv, C. Ying, Z. Hu, H. Hu et al., Facile one-step preparation of laminated PDMS based flexible strain sensors with high conductivity and sensitivity via filler sedimentation. Compos. Sci. Technol. 186, 107933 (2020). https://doi. org/10.1016/j.compscitech.2019.107933
146. D. An, S. Cheng, S. Xi, Z. Zhang, X. Duan et al., Flexible thermal interfacial materials with covalent bond connections for improving high thermal conductivity. Chem. Eng. J. 383, 123151 (2020). https://doi.org/10.1016/j.cej.2019.123151

147. J. Peng, I. Witting, N. Geisendorfer, M. Wang, M. Chang et al., 3D extruded composite thermoelectric threads for flexible energy harvesting. Nat. Commun. 10(1), 5590 (2019). https://doi.org/10.1038/s41467-019-13461-2

148. B. Dorling, J.D. Ryan, J.D. Craddock, A. Sorrentino, A.E. Basaty et al., Photoinduced p- to n-type switching in thermoelectric polymer-carbon nanotube composites. Adv. Mater. 28(14), 2782-2789 (2016). https://doi.org/10.1002/adma. 201505521

149. L. Cai, J. Li, P. Luan, H. Dong, D. Zhao et al., Highly transparent and conductive stretchable conductors based on hierarchical reticulate single-walled carbon nanotube architecture. Adv. Funct. Mater. 22(24), 5238-5244 (2012). https://doi.org/ 10.1002/adfm.201201013

150. Q. Liu, J. Chen, Y. Li, G. Shi, High-performance strain sensors with fish-scale-like graphene-sensing layers for fullrange detection of human motions. ACS Nano 10(8), 79017906 (2016). https://doi.org/10.1021/acsnano.6b03813

151. A.M. Xie, K. Zhang, M.X. Sun, Y.L. Xia, F. Wu, Facile growth of coaxial Ag@polypyrrole nanowires for highly tunable electromagnetic waves absorption. Mater. Design 154, 192-202 (2018). https://doi.org/10.1016/j.matdes.2018.05.039

152. G.J. Li, K. Dai, M.N. Ren, Y. Wang, G.Q. Zheng et al., Aligned flexible conductive fibrous networks for highly sensitive, ultrastretchable and wearable strain sensors. J. Mater. Chem. C 6(24), 6575-6583 (2018). https://doi.org/10.1039/ c8tc01924j

153. L.J. Lu, Y.J. Zhou, J. Pan, T.Q. Chen, Y.J. Hu et al., Design of helically double-leveled gaps for stretchable fiber strain sensor with ultralow detection limit, broad sensing range, and high repeatability. ACS Appl. Mater. Interfaces 11(4), 4345-4352 (2019). https://doi.org/10.1021/acsami.8b17666

154. B. Zhou, Z. Zhang, Y.L. Li, G.J. Han, Y.Z. Feng et al., Flexible, robust, and multifunctional electromagnetic interference shielding film with alternating cellulose nanofiber and MXene layers. ACS Appl. Mater. Interfaces 12(4), 48954905 (2020). https://doi.org/10.1021/acsami.9b19768

155. J.T. Muth, D.M. Vogt, R.L. Truby, Y. Menguc, D.B. Kolesky et al., Embedded 3D printing of strain sensors within highly stretchable elastomers. Adv. Mater. 26(36), 6307-6312 (2014). https://doi.org/10.1002/adma.201400334

156. J. Han, H. Wang, Y. Yue, C. Mei, J. Chen et al., A selfhealable and highly flexible supercapacitor integrated by dynamically cross-linked electro-conductive hydrogels based on nanocellulose-templated carbon nanotubes embedded in a viscoelastic polymer network. Carbon 149, 1-18 (2019). https://doi.org/10.1016/j.carbon.2019.04.029

157. J. Liu, J. Sun, L. Gao, Flexible single-walled carbon nanotubes/polyaniline composite films and their enhanced thermoelectric properties. Nanoscale 3(9), 3616-3619 (2011). https://doi.org/10.1039/c1nr10386e 
158. P.B. Liu, Y. Huang, X. Sun, Excellent electromagnetic absorption properties of poly(3,4-ethylenedioxythiophene)reduced graphene oxide- $\mathrm{Co}_{3} \mathrm{O}_{4}$ composites prepared by a hydrothermal method. ACS Appl. Mater. Interfaces 5(23), 12355-12360 (2013). https://doi.org/10.1021/am404561c

159. B. Zhang, S. Lin, J. Zhang, X. Li, X. Sun, Facile synthesis of sandwich-like $\mathrm{rGO} / \mathrm{CuS} /$ polypyrrole nanoarchitectures for efficient electromagnetic absorption. Materials 13(2), 446 (2020). https://doi.org/10.3390/ma13020446

160. S. Dong, X. Zhang, W. Zhang, J. Han, P. Hu, A multiscale hierarchical architecture of a $\mathrm{SiC}$ whiskers-graphite nanosheets/polypyrrole ternary composite for enhanced electromagnetic wave absorption. J. Mater. Chem. C 6(40), 10804-10814 (2018). https://doi.org/10.1039/c8tc03683g

161. Y.N. Koh, N. Mokhtar, S.W. Phang, Effect of microwave absorption study on polyaniline nanocomposites with untreated and treated double wall carbon nanotubes. Polym. Compos. 39(4), 1283-1291 (2018). https://doi.org/10.1002/ pc. 24064

162. Q. Xu, S. Qu, C. Ming, P. Qiu, Q. Yao et al., Conformal organic-inorganic semiconductor composites for flexible thermoelectrics. Energ. Environ. Sci. 13(2), 511-518 (2020). https://doi.org/10.1039/c9ee03776d

163. T. Nagai, N. Aoki, Y. Ochiai, K. Hoshino, Electric conductivity-tunable transparent flexible nanowire-filled polymer composites: orientation control of nanowires in a magnetic field. ACS Appl. Mater. Interfaces 3(7), 2341-2348 (2011). https://doi.org/10.1021/am200260v

164. O.M. Kwon, H. Watanabe, K.H. Ahn, S.J. Lee, Growths of mechanical elasticity and electrical conductance of graphene nanoplatelet/poly(lactic acid) composites under strong electric field: correlation with time evolution of higher order structure of graphene nanoplatelets. Rheol. Acta 56(11), 871-885 (2017). https://doi.org/10.1007/s00397-017-1042-z

165. X. Huang, K. Wang, K. Jia, X. Liu, Polymer-based composites with improved energy density and dielectric constants by monoaxial hot-stretching for organic film capacitor applications. RSC Adv. 5(64), 51975-51982 (2015). https://doi.org/ $10.1039 / \mathrm{c} 5 \mathrm{ra05029d}$

166. J.R. Bautista-Quijano, P. Pötschke, H. Brünig, G. Heinrich, Strain sensing, electrical and mechanical properties of polycarbonate/multiwall carbon nanotube monofilament fibers fabricated by melt spinning. Polymer 82, 181-189 (2016). https://doi.org/10.1016/j.polymer.2015.11.030

167. G. Wang, G. Zhao, S. Wang, L. Zhang, C.B. Park, Injectionmolded microcellular PLA/graphite nanocomposites with dramatically enhanced mechanical and electrical properties for ultra-efficient EMI shielding applications. J. Mater. Chem. C 6(25), 6847-6859 (2018). https://doi.org/10.1039/c8tc0 $1326 \mathrm{~h}$

168. L. Yang, W. Weng, X. Fei, L. Pan, X. Li et al., Revealing the interrelation between hydrogen bonds and interfaces in graphene/PVA composites towards highly electrical conductivity. Chem. Eng. J. 383, 123126 (2020). https://doi.org/10. 1016/j.cej.2019.123126
169. Y. Guo, L. Pan, X. Yang, K. Ruan, Y. Han et al., Simultaneous improvement of thermal conductivities and electromagnetic interference shielding performances in polystyrene composites via constructing interconnection oriented networks based on electrospinning technology. Compos. Part A Appl. Sci. Manuf. 124, 105484 (2019). https://doi.org/10.1016/j. compositesa.2019.105484

170. X. Hou, Y. Chen, W. Dai, Z. Wang, H. Li et al., Highly thermal conductive polymer composites via constructing microphragmites communis structured carbon fibers. Chem. Eng. J. 375, 121921 (2019). https://doi.org/10.1016/j.cej.2019. 121921

171. H. Liu, M. Dong, W. Huang, J. Gao, K. Dai et al., Lightweight conductive graphene/thermoplastic polyurethane foams with ultrahigh compressibility for piezoresistive sensing. J. Mater. Chem C 5(1), 73-83 (2017). https://doi.org/10.1039/c6tc0 $3713 \mathrm{e}$

172. H. Zhang, G. Zhang, Q. Gao, M. Tang, Z. Ma et al., Multifunctional microcellular PVDF/Ni-chains composite foams with enhanced electromagnetic interference shielding and superior thermal insulation performance. Chem. Eng. J. 379, 122304 (2020). https://doi.org/10.1016/j.cej.2019.122304

173. B. Zhao, J. Deng, C. Zhao, C. Wang, Y.G. Chen et al., Achieving wideband microwave absorption properties in PVDF nanocomposite foams with an ultra-low MWCNT content by introducing a microcellular structure. J. Mater. Chem. C 8(1), 58-70 (2020). https://doi.org/10.1039/c9tc04575a

174. L. Wang, J. Zhang, Y. Guo, X. Chen, X. Jin et al., Fabrication of core-shell structured poly(3,4-ethylenedioxythiophene)/ carbon nanotube hybrids with enhanced thermoelectric power factors. Carbon 148, 290-296 (2019). https://doi.org/ 10.1016/j.carbon.2019.03.088

175. Q. Wang, Q. Yao, J. Chang, L. Chen, Enhanced thermoelectric properties of CNT/PANI composite nanofibers by highly orienting the arrangement of polymer chains. J. Mater. Chem. 22(34), 17612-17618 (2012). https://doi.org/10.1039/c2jm3 $2750 \mathrm{c}$

176. L. Wang, Q. Yao, H. Bi, F. Huang, Q. Wang et al., PANI/ graphene nanocomposite films with high thermoelectric properties by enhanced molecular ordering. J. Mater. Chem. A 3(13), 7086-7092 (2015). https://doi.org/10.1039/c4ta06422d

177. G. Wang, L. Wang, L.H. Mark, V. Shaayegan, G. Wang et al., Ultralow-threshold and lightweight biodegradable porous PLA/ MWCNT with segregated conductive networks for high-performance thermal insulation and electromagnetic interference shielding applications. ACS Appl. Mater. Interfaces 10(1), 1195-1203 (2018). https://doi.org/10.1021/acsami.7b14111

178. C. Liang, P. Song, H. Qiu, Y. Zhang, X. Ma et al., Constructing interconnected spherical hollow conductive networks in silver platelets/reduced graphene oxide foam/epoxy nanocomposites for superior electromagnetic interference shielding effectiveness. Nanoscale 11(46), 22590-22598 (2019). https://doi.org/10.1039/c9nr06022g

179. B. Zhang, J. Sun, H.E. Katz, F. Fang, R.L. Opila, Promising thermoelectric properties of commercial PEDOT: PSS materials and their $\mathrm{Bi}_{2} \mathrm{Te}_{3}$ powder composites. ACS Appl. Mater. 
Interfaces 2(11), 3170-3178 (2010). https://doi.org/10.1021/ am $100654 \mathrm{p}$

180. C.P. Feng, S.S. Wan, W.C. Wu, L. Bai, R.Y. Bao et al., Electrically insulating, layer structured $\mathrm{SiR} / \mathrm{GNPs} / \mathrm{BN}$ thermal management materials with enhanced thermal conductivity and breakdown voltage. Compos. Sci. Technol. 167, 456-462 (2018). https://doi.org/10.1016/j.compscitech.2018.08.039

181. K. Ke, V.S. Bonab, D. Yuan, I. Manas-Zloczower, Piezoresistive thermoplastic polyurethane nanocomposites with carbon nanostructures. Carbon 139, 52-58 (2018). https://doi.org/10. 1016/j.carbon.2018.06.037

182. K. Ke, P. Potschke, N. Wiegand, B. Krause, B. Voit, Tuning the network structure in poly(vinylidene fluoride)/carbon nanotube nanocomposites using carbon black: toward improvements of conductivity and piezoresistive sensitivity. ACS Appl. Mater. Interfaces 8(22), 14190-14199 (2016). https://doi.org/10.1021/acsami.6b03451

183. Y. Lu, Y. Qiu, Q. Jiang, K. Cai, Y. Du et al., Preparation and characterization of Te/poly(3,4-ethylenedioxythiophene): poly(styrenesulfonate) $/ \mathrm{Cu}_{7} \mathrm{Te}_{4}$ ternary composite films for flexible thermoelectric power generator. ACS Appl. Mater. Interfaces 10(49), 42310-42319 (2018). https://doi.org/10. 1021/acsami.8b15252

184. H. Ji, D.P. Sellan, M.T. Pettes, X. Kong, J. Ji et al., Enhanced thermal conductivity of phase change materials with ultrathin-graphite foams for thermal energy storage. Energy. Environ. Sci. 7(3), 1185-1192 (2014). https://doi.org/10. 1039/c3ee42573h

185. P. Miao, K. Cheng, H. Li, J. Gu, K. Chen et al., Poly(dimethylsilylene) diacetylene-guided ZIF-based heterostructures for full Ku-band electromagnetic wave absorption. ACS Appl. Mater. Interfaces 11(19), 17706-17713 (2019). https://doi.org/10.1021/acsami.9b03944

186. S.H. Song, K.H. Park, B.H. Kim, Y.W. Choi, G.H. Jun et al., Enhanced thermal conductivity of epoxy-graphene composites by using non-oxidized graphene flakes with non-covalent functionalization. Adv. Mater. 25(5), 732-737 (2013). https:// doi.org/10.1002/adma.201202736

187. P. Bollen, N. Quievy, C. Detrembleur, J.M. Thomassin, L. Monnereau et al., Processing of a new class of multifunctional hybrid for electromagnetic absorption based on a foam filled honeycomb. Mater. Design 89, 323-334 (2016). https:// doi.org/10.1016/j.matdes.2015.09.129

188. S. Li, A. Huang, Y.J. Chen, D. Li, L.S. Turng, Highly filled biochar/ultra-high molecular weight polyethylene/linear low density polyethylene composites for high-performance electromagnetic interference shielding. Compos. Part B Eng. 153, 277-284 (2018). https://doi.org/10.1016/j.compositesb.2018. 07.049

189. Y. Huang, L. Gao, Y. Zhao, X. Guo, C. Liu et al., Highly flexible fabric strain sensor based on graphene nanoplateletpolyaniline nanocomposites for human gesture recognition. J. Appl. Polym. Sci. 134(39), 45340 (2017). https://doi.org/ 10.1002/app.45340

190. T. Zhang, K. Li, C. Li, S. Ma, H.H. Hng et al., Mechanically durable and flexible thermoelectric films from PEDOT:PSS/
PVA/ $\mathrm{Bi}_{0.5} \mathrm{Sb}_{1.5} \mathrm{Te}_{3}$ nanocomposites. Adv. Electron. Mater. 3(4), 1600554 (2017). https://doi.org/10.1002/aelm.20160 0554

191. Y. Kazemi, A.R. Kakroodi, A. Ameli, T. Filleter, C.B. Park, Highly stretchable conductive thermoplastic vulcanizate/carbon nanotube nanocomposites with segregated structure, low percolation threshold and improved cyclic electromechanical performance. J. Mater. Chem. C 6(2), 350-359 (2018). https://doi.org/10.1039/c7tc04501h

192. H. Bizhani, V. Nayyeri, A. Katbab, A. Jalali-Arani, H. Nazockdast, Double percolated MWCNTs loaded PC/SAN nanocomposites as an absorbing electromagnetic shield. Eur. Polym. J. 100, 209-218 (2018). https://doi.org/10.1016/j. eurpolymj.2018.01.016

193. W. Liu, Y. Yang, M. Nie, Constructing a double-percolated conductive network in a carbon nanotube/polymer-based flexible semiconducting composite. Compos. Sci. Technol. 154, 45-52 (2018). https://doi.org/10.1016/j.compscitech.2017.11. 003

194. Y.D. Li, L.J. Zheng, T.H. Zhao, J. Zhu, J.B. Zeng, Localization control of carbon nanotubes in immiscible polylactide/ vulcanized epoxidized soybean oil blends. Compos. Commun. 11, 6-11 (2019). https://doi.org/10.1016/j.coco.2018. 11.001

195. X. Huang, C. Zhi, P. Jiang, D. Golberg, Y. Bando et al., Polyhedral oligosilsesquioxane-modified boron nitride nanotube based epoxy nanocomposites: an ideal dielectric material with high thermal conductivity. Adv. Funct. Mater. 23(14), 1824-1831 (2013). https://doi.org/10.1002/adfm.201201824

196. K. Wu, P. Liao, R. Du, Q. Zhang, F. Chen et al., Preparation of a thermally conductive biodegradable cellulose nanofiber/ hydroxylated boron nitride nanosheet film: the critical role of edge-hydroxylation. J. Mater. Chem. A 6(25), 11863-11873 (2018). https://doi.org/10.1039/c8ta03642j

197. X. Zeng, J. Sun, Y. Yao, R. Sun, J.B. Xu et al., A combination of boron nitride nanotubes and cellulose nanofibers for the preparation of a nanocomposite with high thermal conductivity. ACS Nano 11(5), 5167-5178 (2017). https://doi.org/10. 1021/acsnano.7b02359

198. S. Liu, S. Xue, S. Xiu, B. Shen, J. Zhai, Surface-modified $\mathrm{Ba}\left(\mathrm{Zr}_{0.3} \mathrm{Ti}_{0.7}\right) \mathrm{O}_{3}$ nanofibers by polyvinylpyrrolidone filler for poly(vinylidene fluoride) composites with enhanced dielectric constant and energy storage density. Sci. Rep. 6, 26198 (2016). https://doi.org/10.1038/srep26198

199. Z. Sang, K. Ke, I. Manas-Zloczower, Interface design strategy for the fabrication of highly stretchable strain sensors. ACS Appl. Mater. Interfaces 10(42), 36483-36492 (2018). https:// doi.org/10.1021/acsami.8b14573

200. Y. Zhan, M. Oliviero, J. Wang, A. Sorrentino, G.G. Buonocore et al., Enhancing the EMI shielding of natural rubberbased supercritical $\mathrm{CO}_{2}$ foams by exploiting their porous morphology and CNT segregated networks. Nanoscale 11(3), 1011-1020 (2019). https://doi.org/10.1039/c8nr07351a

201. Z. Pan, L. Yao, J. Zhai, B. Shen, S. Liu et al., Excellent energy density of polymer nanocomposites containing $\mathrm{BaTiO}_{3} @$ $\mathrm{Al}_{2} \mathrm{O}_{3}$ nanofibers induced by moderate interfacial area. $\mathrm{J}$. 
Mater. Chem. A 4(34), 13259-13264 (2016). https://doi.org/ 10.1039/c6ta05233a

202. Z. Pan, L. Yao, J. Zhai, D. Fu, B. Shen et al., High-energydensity polymer nanocomposites composed of newly structured one-dimensional $\mathrm{BaTiO}_{3} @ \mathrm{Al}_{2} \mathrm{O}_{3}$ nanofibers. ACS Appl. Mater. Interfaces 9(4), 4024-4033 (2017). https://doi. org/10.1021/acsami.6b13663

203. Y. Zhang, Y. Wang, Y. Deng, M. Li, J. Bai, Enhanced dielectric properties of ferroelectric polymer composites induced by metal-semiconductor $\mathrm{Zn}-\mathrm{ZnO}$ core-shell structure. ACS Appl. Mater. Interfaces 4(1), 65-68 (2012). https://doi.org/ 10.1021/am2016156

204. Q. Chi, X. Wang, C. Zhang, Q. Chen, M. Chen et al., High energy storage density for poly(vinylidene fluoride) composites by introduced core-shell $\mathrm{CaCu}_{3} \mathrm{Ti}_{4} \mathrm{O}_{12} @ \mathrm{Al}_{2} \mathrm{O}_{3}$ nanofibers. ACS Sustain. Chem. Eng. 6(7), 8641-8649 (2018). https://doi.org/10.1021/acssuschemeng.8b00941

205. Y. Yang, Z. Chen, X. Song, B. Zhu, T. Hsiai et al., Three dimensional printing of high dielectric capacitor using projection based stereolithography method. Nano Energy 22, 414-421 (2016). https://doi.org/10.1016/j.nanoen.2016.02. 045

206. Y. Li, D. Zhang, S. Wang, Y. Zhan, J. Yin et al., $\mathrm{Fe}_{3} \mathrm{O}_{4}$ decorated graphene/poly(vinylidene fluoride) nanocomposites with high dielectric constant and low dielectric loss. Compos. Sci. Technol. 171, 152-161 (2019). https://doi.org/10.1016/j. compscitech.2018.12.022

207. L. Lin, L. Wang, B. Li, J. Luo, X. Huang et al., Dual conductive network enabled superhydrophobic and high performance strain sensors with outstanding electro-thermal performance and extremely high gauge factors. Chem. Eng. J. 385, 123391 (2020). https://doi.org/10.1016/j.cej.2019.123391

208. Y.J. Yim, K.Y. Rhee, S.J. Park, Electromagnetic interference shielding effectiveness of nickel-plated MWCNTs/high-density polyethylene composites. Compos. Part B Eng. 98, 120-125 (2016). https://doi.org/10.1016/j.compositesb.2016.04.061

209. Y. Lin, S. Liu, S. Chen, Y. Wei, X. Dong et al., A highly stretchable and sensitive strain sensor based on grapheneelastomer composites with a novel double-interconnected network. J. Mater. Chem. C 4(26), 6345-6352 (2016). https:// doi.org/10.1039/c6tc01925k

210. Y. Zhu, H. Yao, P. Jiang, J. Wu, X. Zhu et al., Two-dimensional high- $k$ nanosheets for dielectric polymer nanocomposites with ultrahigh discharged energy density. J. Phys. Chem. C 122(32), 18282-18293 (2018). https://doi.org/10.1021/acs. jpcc.8b04918

211. S. Zhang, H. Deng, Q. Zhang, Q. Fu, Formation of conductive networks with both segregated and double-percolated characteristic in conductive polymer composites with balanced properties. ACS Appl. Mater. Interfaces 6(9), 6835-6844 (2014). https://doi.org/10.1021/am500651v

212. H. Zhou, H. Deng, L. Zhang, Q. Fu, Significant enhancement of thermal conductivity in polymer composite via constructing macroscopic segregated filler networks. ACS Appl. Mater. Interfaces 9(34), 29071-29081 (2017). https://doi.org/ 10.1021/acsami.7b07947

213. Z. Liu, Z. Qian, J. Song, Y. Zhang, Conducting and stretchable composites using sandwiched graphene-carbon nanotube hybrids and styrene-butadiene rubber. Carbon 149, 181-189 (2019). https://doi.org/10.1016/j.carbon.2019.04.037 